\title{
Magnetic field decay of magnetars in supernova remnants
}

\author{
Z. F. Gao ${ }^{1,2,3}$ • Q. H. Peng ${ }^{4} \cdot$ N. Wang ${ }^{1,2} \bullet$ \\ J. P. Yuan ${ }^{1,2}$
}

\begin{abstract}
In this paper, we modify our previous research carefully, derive a new expression of electron energy density in superhigh magnetic fields. Based on our improved model, we re-compute the electron capture rates and the magnetic fields' evolutionary timescales $t$ of magnetars. According to the calculated results, the superhigh magnetic fields may evolve on timescales $\sim\left(10^{6}-10^{7}\right)$ yrs for common magnetars, and the maximum timescale of the field decay, $t \approx 2.9507 \times 10^{6} \mathrm{yrs}$, corresponding to an initial internal magnetic field $B_{0}=$ $3.0 \times 10^{15} \mathrm{G}$ and an initial temperature $T_{0}=2.6 \times 10^{8} \mathrm{~K}$. Motivated by the results of the neutron star-supernova remnant(SNR) association of Zhang \& Xie(2011), we calculate the maximum $B_{0}$ of magnetar progenitors, $B_{\max } \sim\left(2.0 \times 10^{14}-2.93 \times 10^{15}\right) \mathrm{G}$ when $T_{0}=2.6 \times 10^{8}$ $\mathrm{K}$. When $T_{0} \sim 2.75 \times 10^{8}-1.75 \times 10^{8} \mathrm{~K}$, the maximum $B_{0}$ will also be in the range of $\sim 10^{14}-10^{15} \mathrm{G}$, not exceeding the upper limit of magnetic field of a mag-
\end{abstract}

\footnotetext{
Z. F. Gao

${ }^{1}$ Xinjiang Astronomical Observatory, CAS, 150, Science 1-Street, Urumqi Xinjiang, 830011,China zhifu_gao@uao.ac.cn

${ }^{2}$ Key Laboratory of Radio Astronomy, Chinese Academy of SciencesNanjing, 210008, China

${ }^{3}$ Graduate University of the Chinese Academy of Sciences, 19A Yuquan Road, Beijing, 100049, China

Q. H. Peng

${ }^{4}$ Department of Astronomy, Nanjing University, Nanjing, 210093. China

N. Wang

${ }^{1}$ Xinjiang Astronomical Observatory, CAS, 40-5 South Beijing Road, Urumqi Xinjiang, 830011, China

${ }^{2}$ Key Laboratory of Radio Astronomy, Chinese Academy of SciencesNanjing, 210008, China

J. P. Yuan

${ }^{1}$ Xinjiang Astronomical Observatory, CAS, 40-5 South Beijing Road, Urumqi Xinjiang, 830011, China

${ }^{2}$ Key Laboratory of Radio Astronomy, Chinese Academy of SciencesNanjing, 210008, China
}

netar under our magnetar model. We also investigate the relationship between the spin-down ages of magnetars and the ages of their SNRs, and explain why all AXPs associated with SNRs look older than their real ages, whereas all SGRs associated with SNRs appear younger than they are.

Keywords Magnetar. Electron capture rate. Supernova remnant. and Superhigh magnetic field

\section{Introduction}

Recent developments have shown that a substantial fraction of newly born stars have magnetic field strengths in excess of the quantum critical value, $B_{\mathrm{cr}}=4.414 \times 10^{13} \mathrm{G}$, above which the effect of Landau quantization on the transverse electron motion becomes considerable(Ternov et al. 1965). We divide them into two glasses - Soft Gamma-ray Repeaters(SGRs) and Anomalous X-ray Pulsars (AXPs) through the studies of their emission mechanisms. The SGRs and AXPs are currently considered to be 'magnetars', powered by extremely strong magnetic fields, rather than by their spin-down energy loss, as is the case for common radio pulsars (Duncan \& Thompson 1992; Thompson \& Duncan 1993; Thompson \& Duncan 1996).

A supernova remnant(SNR) is an expanding diffuse gaseous nebula that results from the explosion of a massive star. To date, there are 23 detected magnetar candidates: 11 SGRs (7 confirmed), and 12 AXPs (9 confirmed). Of the magnetar candidates, 4 SGRs and 5 AXPs (more than a third) are associated with the known SNRs, suggestive of an origin in massive star explosions (Gaensler et al. 2001; Marsden et al. 2001; Allen \& Horvath 2004; Mereghetti 2008). Estimations indicate that about $10 \%$ of supernova explosions may 
lead to a magnetar (Kouveliotou et al. 1994). If magnetars are from core-collapse supernovae, as estimated above, then their magnetic fields could have been inherited from their progenitors (Horiuchi et al. 2008).

The SGRs, characterized primarily by their occasional repeating bursts of soft $\gamma$-rays, have spin periods $(5 \sim 8)$ s, positive period derivatives, and persistent soft X-ray luminosities $\sim 10^{35} \mathrm{erg} \mathrm{s}^{-1}$ (Duncan 2000; Marsden et al. 2001). There are four known SGRs associated with SNRs: O526-66, 1900+14, 180620 and 1627-41. SGR 0526-66 has been associated with N49 in the Large Magellanic Cloud Kulkarni et al. 2003). SGR 1806-20 and SGR 1627-41 apparently lie in G10.0-0.3 (Kulkarni \& Frail 1993) and G33.70-01 (Corbel et al. 1999), respectively. SGR $1900+14$ is associated with G42.8+0.6 (Hurley et al. 1999). However, another SNR G43.9+1.6 also falls within the error box of SGR 1900+14 (Vasisht et al. 1994), so a great deal of effort will be required to investigate whether this magnetar is stably associated with G42.8+0.6.

The AXPs, so called due to their high X-ray luminosities, $10^{34} \sim 10^{36}$ erg $\mathrm{s}^{-1}$, the lack of evidence of binary companions van Paradijs et al. 1995; Duncan \& Thompson 1996). To date, there are five AXPs are located near the centers of SNRs: 1E 2259+586 in CTB 109 (Fahlman \& Gregorv 1981), 1E 1841-045 in Kes 73 (Sanbonmatsu \& Herfand 1992), 1E 1547.0-5408 in G327.24-0.13 (Camilo et al. 2007); CXOU J171405.7-381031 in CTB37B (Aharonian et al. 2008) and AX J1845-0258 in G29.6+0.1 (Gaensler et al. 1999).

Due to a very little number of magnetars (only 16 confirmed currently), some puzzles concerning supernova progenitors and their birth events that confront us seem inevitable. However, a few confirmable magnetar/SNR associations still remain undisputed Gaensler et al. 2001; Marsden et al. 2001; Allen \& Horvath 2004). These associations are confirming that a magnetar candidate was formed in a supernova explosion, and is thus thought to be the collapsed core of a massive star, which is likely to be a neutron star(NS). Since a NS and its associated SNR are from the same explosion, they should have the same age (Zhang \& Xie 2011). All the known SNRs of both AXPs and SGRs are comparatively young, $t_{\mathrm{SNR}} \sim$ several kyr (see Sec.3), which infers that magnetars are also very young objects (Shull et al. 1989; Vasisht \& Gotthelf 1997).

Over the last decades, radio pulsars(here, observed as normal radio pulsars) with the possible magnetic field evolutionary timescale, $t \sim 10^{6}-10^{7} \mathrm{yrs}$, have been studied extensively using the statistical distribution in the $P-\dot{P}$ diagram Ostriker \& Gunn 1969; Gunn \& Ostriker|1970; Shull et al. 1989; Narayan \& Ostriker_nption on the origin of superhigh magnetic fields
1990; Han 1997; Ibrahim et al. 2004; Aguilera et al. 2008; Ridley \& Lorimer 2010; Zhang \& Xie 2011). Maybe radio pulsars were born with different initial external circumstances and initial internal conditions, these pulsars have experienced different evolutionary routes: the magnetic fields remain nearly constant in about half of them, decrease rapidly in others, or increase in very few pulsars (Han 1997; Zhang \& Xie 2011).

In order to explain the evolution of superhigh magnetic fields inside magnetars, different models have been proposed recently, as partly listed below. According to the twisted magnetospheres model, the unwinding of the internal field shears the star's crust, the rotational crustal motions generally provide a source of helicity for the external magnetosphere by twisting the magnetic fields which are anchored to the star's surface, drive currents outside a magnetar, and generate X-ray emissions (Thompson et al. 2000). The sudden crustal fractures (or starquakes) caused by unbearable stress can provide plausible mechanisms for magnetar outbursts and giant flares (Thompson et al. 2000, 2002). In addition, the overall evolution of SGR $1806-20$ in the years preceding the giant flare of December 27, (Mereghetti et al. 2005).

In the thermal evolution model, the field could decay directly as a result of the non-zero resistivity of the matter through Ohmic decay or ambipolar diffusion, or indirectly as a result of Hall drift producing a cascade of the field to high wave number components, which decay quickly through Ohmic decay Goldreich \& Reisenegger 1992; Rheinhardt \& Geppert 2003; Pons et al. 2006); magnetic field decay can be a main source of internal heating (Pons et al. 2009; Arras et al. 2004); the enhanced thermal conductivity in the strongly magnetized envelope contributes to ise the surface temperature (Heyl \& Hernquist 1997; Hevl \& Kulkarni 1998). Based on this model, the surface thermal temperature of a magnetar is estimated to be $\sim\left(10^{5}-10^{6}\right) \mathrm{K}$, which is basically consistent with the observations Heyl \& Kulkarni 1998; Pons et al. 2009).

Although there are apparent advantages in some magnetar models, including the above two magnetar models, substantial improvements must be made for these models. The main disadvantages of these models can be summarized as follows: 1 . not considering the effects of anisotropic ${ }^{3} P_{2}$ neutron superfluid (mainly in the outer core) on the decay of superhigh magnetic fields; 2 . not combining the ages of SNRs with the timescales of magnetic fields' evolution; 3. universally assuming the most popular as2004 seems to support some predictions of this model 
of magnetars-' $\alpha-\Omega$ dynamo' (Duncan \& Thompson 1992; Thompson \& Duncan 1993), which is a mere assumption laking observational support (Gao et al. 2011a,b.d.d) (hereinafter Paper 1, Paper 2, Paper 3 and Paper 4, respectively).

Unlike other magnetar models, we propose that superhigh magnetic fields of magnetars originate from the induced magnetic fields below a critical temperature, and the maximum field strength is $\sim(3.0-4.0) \times 10^{15}$ G (Peng \& Tong 2007; Peng \& Tong 2009). In the initial stage of our magnetar model, the major conclusions are briefly summarized as following: In Paper 1, we numerically simulated the whole process of electron capture (EC); in order to calculate the effective electron capture rates, $\Gamma_{\text {eff }}$, we introduced the Landau level effect coefficient, $q$, whose magnitude is evaluated to be $\sim 10^{-18}$ by comparing the observed magnetar's soft X-ray luminosity $L_{\mathrm{X}}$. In Paper 2, superhigh magnetic fields give rise to a increase in the electron Fermi energy $E_{\mathrm{F}}(\mathrm{e})$, which will induce EC inside a magnetar. The ${ }^{3} P_{2}$ Cooper pairs with the maximum binding energy of $0.048 \mathrm{MeV}$ (Elgarøy et al. 1996) will be destroyed by the outgoing high-energy EC neutrons. Then the magnetic moments of the ${ }^{3} P_{2}$ Cooper pairs destroyed are no longer arranged in the paramagnetic direction, so the superhigh magnetic fields produced by the aligned magnetic moments of the ${ }^{3} P_{2}$ Cooper pairs will disappear gradually. Combining parameter $q$ with ${ }^{3} P_{2}$ anisotropic neutron superfluid theory yields a second-order differential equation for superhigh magnetic fields $B$ and their evolution timescales $t$. In Paper 3 , by introducing the Dirac $\delta$-function, we deduced a general formula for $E_{\mathrm{F}}(\mathrm{e})$, which is suitable for extremely intense magnetic fields. In Paper 4, we presented the mechanism foe the magnetar soft $\mathrm{X} / \Gamma$-ray emission, numerically simulated the process of magnetar cooling and magnetic field decay, and then computed $L_{\mathrm{X}}$ of magnetars by introducing two important parameters: Landau level-superfluid modified factor $\Lambda$ and effective $\mathrm{X} / \gamma$-ray coefficient $\zeta$.

In this work, we re-examine our previous research carefully, find that the expression of energy state density $\rho_{\mathrm{e}}$ of electrons in superhigh magnetic fields, as that of $E_{\mathrm{F}}(\mathrm{e})$, should be derived in circular cylindrical coordinates rather than in spherical coordinates, because the Landau column becomes a very long and narrow cylinder along the magnetic field. In Appendix B of this article, we modify the expression of $E_{\mathrm{F}}(\mathrm{e})$, derive a new formula of electron energy state density in superhigh magnetic fields, improve the calculated results of $L_{\mathrm{X}}$ and $\zeta$, and compare these results with those of Paper 4. Apart from the modifications above, the expression of $\Gamma$, together with the second-order differential equation for $B$ and $t$ of Paper 2, must be improved.
The main reasons are as follows:

1. In the interior of a magnetar, the process of EC is a relatively independent process, and is irrelevant to the luminosity $L_{\mathrm{X}}$, the values of $\Gamma$ are completely determined by inner physical properties, eg., magnetic field strength, density, temperature, neutron superfluid and so on.

2. Since the magnitude of $q$ can be estimated by the observed luminosities $L_{\mathrm{X}}$ (see Paper 1), in fact, $q$ have included the influences of all the following factors: neutron superfluid's restraining effect, the fractions of all particles participating in EC reaction, thermal energy loss, energy conversion efficiency, and gravitation redshift, accidentally. However, if the influences of the above factors are considered, the real value of $q$ will be far less than that of Paper 1, therefore, $q$ is no longer used by our improved model. In Paper 4, $q$ has been replaced by two important parameters: Landau level-superfluid modified factor, $\Lambda$, and effective $\mathrm{X} / \gamma$-ray coefficient, $\zeta$ when calculating $L_{\mathrm{X}}$.

3. In our previous work (Papers 1-4), all the expressions of $\Gamma$, however, do not make use of the quantity $\Lambda$ and the new expression of $\rho_{\mathrm{e}}$ in superhigh magnetic fields (see Appendix B), so these expressions of $\Gamma$ are not consistent with the actual circumstances inside magnetars. All of these strongly suggest a necessity of reconsidering $\Gamma$ and the equation of $B$ and $t$.

In 2011, Shuang-Nan Zhang and Yi Xie published an article titled 'Magnetic field decay makes NSs look older than they are', which showed convincing evidence of magnetic field decay in some young NSs, and reasoned that the magnetic field decay can change substantially their spinning behaviors such that these NSs appear much older than their real ages Zhang \& Xie 2011) (hereinafter referred to as ZX2011). According to ZX2011, a NS and its associated SNR should have the same ages, ie., $t_{\text {Real }}=t_{\mathrm{SNR}}$; the NS's spin-down or characteristic age can be generally expressed as, $t_{\text {Spin }}=P /(n-1) \dot{P}$, where $P, \dot{P}$ and $n$ are its spin period, period derivative and braking index, respectively; however, $n$ always deviates from the the value $(n=3)$ expected for pure magnetic dipole radiation model. The authors supposed that, $n \gg 3$ is required for neutrons with $t_{\text {Spin }} \ll t_{\mathrm{SNR}}$ if there is no significant magnetic field decay (note: this case is not believed to be plausible by authors), $n<3$ makes the spin-down age of a NS even longer than assuming $n=3$, and in this case more or even all all NSs have $t_{\text {Spin }} \ll t_{\mathrm{SNR}}$; for any reasonable values of $n$, at least some of these NSs must have experienced significant dipole magnetic 
field decay. Furthermore, magnetic field decay dominated by the ambipolar diffusion has been investigated, and the core and surface temperatures of a NS have been estimated, whose results are agreed qualitatively with observations (Pons et al. 2009). However, authors did not provide the observation data of magnetars, and thus omitted to explain why all AXPs associated with SNRs look older than their real ages, whereas all SGRs associated with SNRs appear younger than they are.

The remainder of this paper is organized as follows. In Sec.2, by introducing two different types of electron energy state density, the electron capture rates $\Gamma$ in superhigh magnetic fields are calculated, and the calculated results are compared. In Sec.3.1, the differential equation of $B$ and $t$ is modified, and the values of $t$ are re-computed. In Sec.3.2, the maximum initial internal fields of magnetar progenitors are computed taking $t_{\text {Real }}=t_{\text {SNR }}$ proposed by ZX2011 as the starting point. In Sec.3.3, the relationship between the spin-down ages of magnetars and the ages of their SNRs are investigated. In Sec.4, a brief summary is given. In Appendix $\mathrm{A}$, an important assumption on the ${ }^{3} P_{2}$ neutron Cooper pairs is presented, and several corrections and improvements in our models are presented in Appendix B.

\section{Electron capture rates in superhigh magnetic fields}

Since the quantized microstates don't exist in the momentum (or energy) space between the $n$-th and $(n+1)$ th Landau level, the Dirac $\delta$-function must be taken into account when calculating $E_{\mathrm{F}}(\mathrm{e})$ in superhigh magnetic fields. From Appendix B, a concise expression for $E_{\mathrm{F}}(\mathrm{e})$ in superhigh magnetic fields is of the form,

$E_{\mathrm{F}}(\mathrm{e}) \simeq 43.44\left(\frac{B}{B_{\mathrm{cr}}}\right)^{\frac{1}{4}}\left(\frac{\rho}{\rho_{0}} \frac{Y_{\mathrm{e}}}{0.0535}\right)^{\frac{1}{4}} \quad \mathrm{MeV}$.

where $\rho_{0}=2.8 \times 10^{14} \mathrm{~g} \mathrm{~cm}^{3}$ is the standard nuclear density. In order to calculate the $\mathrm{EC}$ rate, $\Gamma$ in a magnetar, we concentrate on non-relativistic, degenerate nuclear matter and super-relativistic, degenerate electrons. In the case of $0.5 \rho_{0} \leq \rho \leq 2 \rho_{0}$, the following expressions hold approximately: $E_{\mathrm{F}}^{\prime}(\mathrm{n})=$ $60\left(\rho / \rho_{0}\right)^{\frac{2}{3}} \mathrm{MeV}$ and $E_{\mathrm{F}}^{\prime}(\mathrm{p})=1.9\left(\rho / \rho_{0}\right)^{\frac{4}{3}} \mathrm{MeV}$, where $E_{\mathrm{F}}^{\prime}(\mathrm{n})$ and $E_{\mathrm{F}}^{\prime}(\mathrm{p})$ are the neutron Fermi kinetic energy and the proton Fermi kinetic energy, respectively (Shapiro \& Teukolsky 1983). In this paper, for convenience, we set $\rho=\rho_{0}$ and the electron fraction $Y_{\mathrm{e}}=0.0535$ in all the following calculations. This choice yields the threshold energy of EC reaction, $Q=E_{\mathrm{F}}(\mathrm{n})-E_{\mathrm{F}}(\mathrm{p})=59.39 \mathrm{MeV}$. The range of $B$ is assumed to be $B \sim\left(B_{\mathrm{th}} \sim 3.0 \times 10^{15} \mathrm{G}\right)$, where
$B_{\mathrm{th}}=1.5423 \times 10^{14} \mathrm{G}$ is the threshold magnetic field of EC reaction, corresponding to $E_{F}(\mathrm{e})=59.39 \mathrm{MeV}$. Thus, the range of $E_{\mathrm{e}}$ is $\left(Q \sim E_{\mathrm{F}}(\mathrm{e})\right)$. By employing energy conservation via $E_{\nu}+E_{\mathrm{n}}=E_{\mathrm{e}}+E_{\mathrm{p}}$, the Fermi energy of neutrinos, $E_{\mathrm{F}}(\nu)$, can be calculated by

$$
E_{\mathrm{F}}(\nu)=E_{\mathrm{F}}(\mathrm{e})-Q=E_{\mathrm{F}}(\mathrm{e})-59.39 \quad \mathrm{MeV} .
$$

According to our point of view, once the energies of electrons near the Fermi surface exceed $Q$, the EC reaction will dominate (see Paper 2 and Paper 4). From Appendix B, the energy state density of electrons in superhigh magnetic fields is of the form:

$$
\begin{aligned}
& \rho_{\mathrm{e}} \simeq \frac{4}{3} \frac{\pi}{B^{*}}\left(\frac{m_{\mathrm{e}} c}{h}\right)^{3} \frac{1}{m_{\mathrm{e}} c^{2}}\left[\left(\frac{E_{F}(\mathrm{e})}{m_{\mathrm{e}} c^{2}}\right)^{2}-1-\left(\frac{E_{\mathrm{e}}}{m_{\mathrm{e}} c^{2}}\right)^{2}\right]^{\frac{3}{2}} \\
& =\frac{1}{3 B^{*}\left(2 \pi^{2} \hbar^{3} c^{3}\right)} \frac{1}{m_{\mathrm{e}} c^{2}}\left[E_{F}^{2}(\mathrm{e})-0.261-E_{\mathrm{e}}^{2}\right]^{\frac{3}{2}} \cdot(3)
\end{aligned}
$$

where $B^{*}$ is a non-dimensional magnetic field, defined as $B^{*}=B / B_{\mathrm{cr}}$. Since neutrinos/antineutrinos are uncharged, the energy state density of neutrinos (antineutrinos) remains unchanged,

$\rho_{\nu}=\frac{\left(E_{\mathrm{e}}-Q\right)^{2}}{2 \pi^{2} \hbar^{3} c^{3}}$.

The EC rate $\Gamma$, defined as the number of electrons captured by one proton per second, can be computed using the standard charged-current $\beta$-decay theory (Shapiro \& Teukolsky 1983). However, for each degenerate species, only a fraction $\left(\sim k T / E_{\mathrm{F}}(\mathrm{i})\right)$ of particles near the Fermi surface can effectively contribute to $\Gamma$. In Paper 4, we introduced the 'Landau levelsuperfluid modified factor' $\Lambda$,

$\Lambda=\frac{(k T)^{4} \operatorname{Exp}\left(-\Delta_{\max }\left({ }^{3} P_{2}\right) / k T\right)}{E_{\mathrm{F}}^{\prime}(\mathrm{n}) E_{\mathrm{F}}^{\prime}(\mathrm{p}) E_{\mathrm{F}}(\mathrm{e}) E_{\mathrm{F}}\left(\nu_{\mathrm{e}}\right)}$.

The initial conditions (e.g., $B, T$ ) of the magnetars are likely to be very different, implying that their values of $\Lambda$ are also different. For convenience, we assume a uniform initial magnetic field $B_{0}=3.0 \times 10^{15} \mathrm{G}$ for magnetar progenitors. Since the process of EC is a precess of magnetic field decay and inner cooling, when electrons are captured, the numbers of particles participating in EC near the Fermi surfaces decrease, which leads to a decrease in $\Lambda$. However, in the interior of a magnetar, the $\beta$ - decay and the inverse $\beta$-decay occur simultaneously as required by the charge neutrality, so when B decays, the depleted protons and electrons are recruited many times, which leads to only a small decrease in $Y_{\mathrm{e}}$ and $Y_{\mathrm{p}}$. In addition, the electrons are super-relativistic and degenerate, when the internal temperature falls, the electron transition between Landau levels is not permitted, because the electrons can 
be approximately treated as a zero-temperature Fermi gas. Thus, the value of $\Lambda$ decreases very slowly. In order to obtain a fitting function of $\Lambda, B$ and $T$, we numerically simulate the inner cooling and the magnetic field decay. Since the internal temperature of a magnetar is $\sim 10^{8} \mathrm{~K}$ (Yakovlev et al. 2001), and the maximum initial temperature (not including the inner core temperature) cannot exceed the critical temperature of the ${ }^{3} P_{2}$ Cooper pairs $T_{\text {cn }} \sim \Delta_{\max }\left({ }^{3} P_{2}\right) / k T \sim 2.78 \times 10^{8} \mathrm{~K}(\mathrm{~Pa}-$ per 4 ), we can arbitrarily assume $T_{0}$ to be $2.60 \times 10^{8} \mathrm{~K}$, corresponding to an initial value of $\Lambda \sim 3.198 \times 10^{-14}$. Then, we gain

$$
\begin{aligned}
& \Lambda\left(B_{0}, T_{0}\right)=\Lambda\left(T_{0}\right)=3.198 \times 10^{-14}\left(\frac{T_{0}}{2.6 \times 10^{8} \mathrm{~K}}\right)^{4} \\
& \operatorname{Exp}\left[\frac{-0.048 \mathrm{MeV}}{k}\left(\frac{1}{T_{0}}-\frac{1}{2.6 \times 10^{8} \mathrm{~K}}\right)\right] .
\end{aligned}
$$

When $B$ decreases from $B_{0}$ to $B_{\text {th }}$, the ratio of $\left|\frac{\Delta T}{\Delta B}\right|_{\max } \sim\left|\frac{\left(2.78 \times 10^{8}-1.0 \times 10^{8}\right) \mathrm{K}}{\left(3.0 \times 10^{15}-1.5423 \times 10^{14}\right) \mathrm{G}}\right| \sim 5.33 \times 10^{-8} \mathrm{~K} / \mathrm{G}$. When simulating numerically, the assumed value of $\left|\frac{\Delta T}{\Delta B}\right|$ cannot be too high or too small, otherwise the internal temperature $T$ drops wildly (eg., $T \ll 10^{7} \mathrm{~K}$ ) or insignificantly. According to the analysis above, we arbitrarily set $B_{0}=3.0 \times 10^{15}, T_{0}=2.60 \times 10^{8} \mathrm{~K}$ and $\Lambda\left(B_{0}, T_{0}\right)=3.198 \times 10^{-14}$ (these specific values are representative of the initial conditions encountered), and $T$ is decreased by step $\Delta T=3.5 \times 10^{4} \mathrm{~K}$. The results of numerical simulations are shown in Fig.1. From Fig. 1 a and Fig.1 b, both $\Lambda$ and $T$ decrease with decreasing $B$. When $\left.B \sim 3.0 \times 10^{15}-2.0 \times 10^{14}\right) \mathrm{G}$, $\Lambda \sim 3.198 \times 10^{-14}-2.649 \times 10^{-14}$. Base on the simulations above we gain

$$
\begin{aligned}
& \Lambda(B, T) \approx\left(2.60388 \times 10^{-14}+1.98233\right. \\
& \left.\times 10^{-30} B\right)\left(\frac{T_{0}}{2.6 \times 10^{8} \mathrm{~K}}\right)^{4} \operatorname{Exp}\left[\frac{-0.048 \mathrm{MeV}}{k}\right. \\
& \left.\left(\frac{1}{T_{0}}-\frac{1}{2.6 \times 10^{8} \mathrm{~K}}\right)\right],
\end{aligned}
$$

where $B_{0}=3.0 \times 10^{-15} \mathrm{G}$ is used. In the same way, we obtain a diagram of $\Lambda$ as a function of internal temperature $T$, as shown in Fig.2. From Fig.2, when internal temperature $T$ drops from $2.60 \times 10^{8} \mathrm{~K}$ to $1.52 \times 10^{8}$ $\mathrm{K}, \Lambda$ decreases, but its order of magnitude remains unchanged.

Using Eq.(7), we obtain the expression of $\Gamma$ in superhigh magnetic fields,

$$
\begin{aligned}
& \Gamma=\Lambda(B, T) \frac{2 \pi}{\hbar} G_{\mathrm{F}}^{2} C_{\mathrm{V}}^{2}\left(1+3 a^{2}\right) \int_{Q}^{E_{F}(\mathrm{e})} f_{\mathrm{e}}\left(1-f_{\nu}\right) \\
& \times f_{\mathrm{p}}\left(1-f_{\mathrm{n}}\right) \rho_{\nu} \rho_{\mathrm{e}} d E_{\mathrm{e}} \rho_{\nu} d E_{\nu} \delta\left(E_{\nu}+Q-E_{\mathrm{e}}\right) \\
& =\Lambda \frac{2 \pi G_{F}^{2} C_{V}^{2}\left(1+3 a^{2}\right)}{\hbar\left(2 \pi^{2} \hbar^{3} c^{3}\right)^{2} 3 m_{\mathrm{e}} c^{2}} \frac{1}{B^{*}} \int_{Q}^{E_{F}(\mathrm{e})} f_{\mathrm{e}}\left(1-f_{\nu}\right) f_{\mathrm{p}} \\
& \left.\times\left(1-f_{\mathrm{n}}\right)\left[E_{F}^{2}(\mathrm{e})-0.261-E_{\mathrm{e}}^{2}\right]^{\frac{3}{2}}\left(E_{\mathrm{e}}-Q\right)^{2}\right) d E,(8)
\end{aligned}
$$

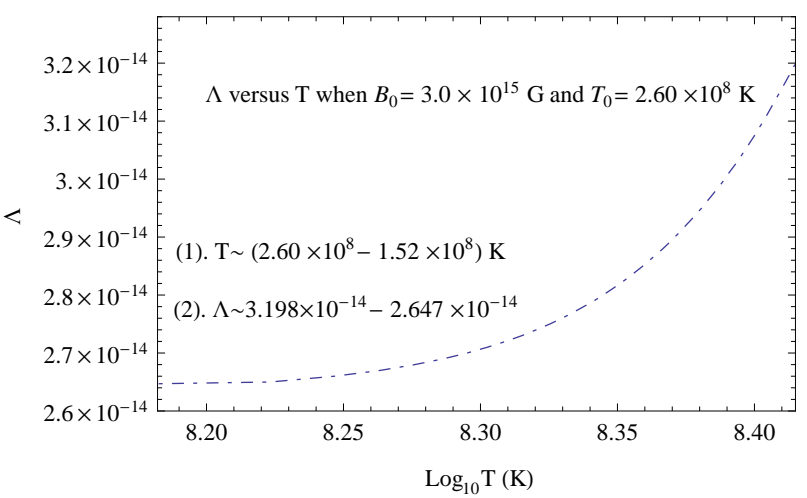

Fig. $2 \Lambda$ as a function of internal temperature $T$. The range of $T$ is about $\left(2.60 \times 10^{8}-1.52 \times 10^{8}\right) \mathrm{K}$ when $B_{0}=$ $3.0 \times 10^{15} \mathrm{G}$.

where $f(\mathrm{j})=\left[\operatorname{Exp}\left(\left(E_{\mathrm{j}}-\mu_{\mathrm{j}}\right) / k T\right)+1\right]^{-1}$ is the fraction of phase space occupied at energy $E_{\mathrm{j}}$ (Fermi-Dirac distribution), factors of $\left(1-f_{\mathrm{j}}\right)$ reduce the reaction rate, and are called 'blocking factor', inside a NS, for neutrinos(antineutrinos), $\left(1-f_{\nu}\right)=1$; for electrons, when $E_{\mathrm{e}}<E_{\mathrm{F}}(\mathrm{e}), f_{\mathrm{e}}=1$, when $E_{\mathrm{e}}>E_{\mathrm{F}}(\mathrm{e}), f_{\mathrm{e}}=$ 0 ; for neutrons, when $E_{k}(\mathrm{n})<E_{\mathrm{F}}^{\prime}(\mathrm{n}),\left(1-f_{\mathrm{n}}\right)=0$, when $E_{k}(\mathrm{n})>E_{\mathrm{F}}^{\prime}(\mathrm{n}),\left(1-f_{\mathrm{n}}\right)=1$; for protons: when $E_{\mathrm{p}}<E_{\mathrm{F}}(\mathrm{p}), f_{\mathrm{p}}=1$, when $E_{\mathrm{p}}>E_{\mathrm{F}}(\mathrm{p}), f_{\mathrm{p}}=0$, so $f_{\mathrm{e}}\left(1-f_{\nu}\right) f_{\mathrm{p}}\left(1-f_{\mathrm{n}}\right) \simeq 1$ can be ignored in the latter calculations; the other quantities have been defined in Paper1, Paper 2 and Paper 4. Eq.(6) is the very expression of $\Gamma$ we have been looking for. Compared with Eqs.(3-4) of Paper 2, the advantages of Eq.(6) mainly includes: 1. It reflects the effective or actual capture rates of electrons in superhigh magnetic fields, which can be calculated directly using Eq.(6), rather than be modified by parameter $q$; 2.It adopts the updated expressions of $\rho_{\mathrm{m}}$ and $E_{\mathrm{F}}(\mathrm{e})$ in superhigh magnetic fields, both of which are derived in circular cylindrical coordinates. In order to compare the results of $\Gamma$ in superhigh magnetic fields calculated by two different types of electron energy state density, we appeal to the following expression,

$$
\begin{aligned}
& \Gamma^{\prime}=\Lambda(B, T) \frac{2 \pi}{\hbar} G_{\mathrm{F}}^{2} C_{\mathrm{V}}^{2}\left(1+3 a^{2}\right) \int_{Q}^{E_{\mathrm{F}}(\mathrm{e})} f_{\mathrm{e}}\left(1-f_{\nu}\right) \\
& \times f_{\mathrm{p}}\left(1-f_{\mathrm{n}}\right) \rho_{\mathrm{e}} d E_{\mathrm{e}}^{\prime} \rho_{\nu} d E_{\nu} \delta\left(E_{\nu}+Q-E_{\mathrm{e}}\right) \\
& =\Lambda \frac{2 \pi}{\hbar} \frac{G_{\mathrm{F}}^{2} C_{\mathrm{V}}^{2}\left(1+3 a^{2}\right)}{\left(2 \pi^{2} \hbar^{3} c^{3}\right)^{2}} \int_{Q}^{E_{\mathrm{F}}(e)} f_{\mathrm{e}}\left(1-f_{\nu}\right) \\
& \times f_{\mathrm{p}}\left(1-f_{\mathrm{n}}\right)\left(E_{\mathrm{e}}^{2}-m_{\mathrm{e}}^{2} c^{4}\right)^{\frac{1}{2}} E_{\mathrm{e}}\left(E_{\mathrm{e}}-Q\right)^{2} d E_{\mathrm{e}}
\end{aligned}
$$

where the most common and typical expression for electron energy state density $\rho_{\mathrm{e}}^{\prime}$ in a sphere symmetrical momentum space, $\rho_{\mathrm{e}}^{\prime}=\frac{4 \pi p_{\mathrm{e}}^{2}}{h^{3}} \frac{d p_{\mathrm{e}}}{d E_{\mathrm{e}}}=\frac{4 \pi p_{\mathrm{e}} E_{\mathrm{e}}}{c^{2} h^{3}}$, is 


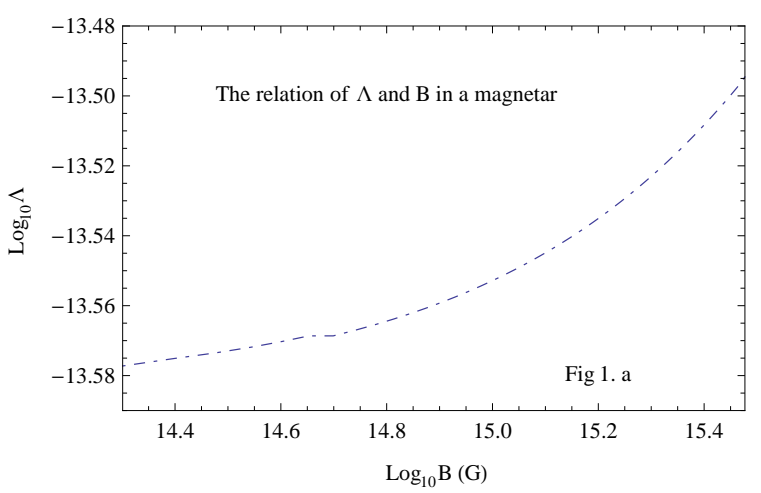

(a)

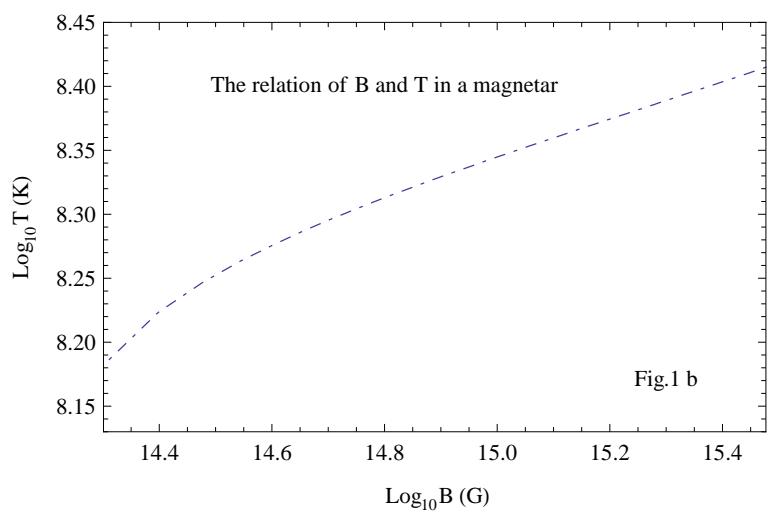

(b)

Fig. $1 \Lambda$ as a function of magnetic field $B$. The range of $B$ is about $\left(3.0 \times 10^{15}-2.0 \times 10^{14}\right) \mathrm{G}$ when $T_{0}=2.60 \times 10^{8} \mathrm{~K}$.

Table 1 The calculated values of electron capture rates in superhigh magnetic fields

\begin{tabular}{crrrrrrr}
\hline $\begin{array}{c}B \\
(\mathrm{G})\end{array}$ & $\begin{array}{r}E_{\mathrm{F}}(\mathrm{e}) \\
(\mathrm{MeV})\end{array}$ & $\begin{array}{r}E_{\mathrm{F}}(\nu) \\
(\mathrm{MeV})\end{array}$ & $\begin{array}{r}\left\langle E_{\mathrm{n}}\right\rangle \\
(\mathrm{MeV})\end{array}$ & $\begin{array}{r}\left\langle E_{\nu}\right\rangle \\
(\mathrm{MeV})\end{array}$ & $\begin{array}{r}\Gamma \\
\left(\mathrm{s}^{-1}\right)\end{array}$ & $\begin{array}{r}\Gamma^{\prime} \\
\left(\mathrm{s}^{-1}\right)\end{array}$ & $K=\Gamma / \Gamma^{\prime}$ \\
\hline $1.8 \times 10^{14}$ & 61.73 & 2.34 & 61.06 & 1.28 & $2.43 \times 10^{-13}$ & $7.76 \times 10^{-12}$ & $3.14 \times 10^{-2}$ \\
$2.0 \times 10^{14}$ & 63.38 & 3.99 & 61.81 & 2.18 & $2.50 \times 10^{-12}$ & $4.01 \times 10^{-11}$ & $6.24 \times 10^{-2}$ \\
$2.5 \times 10^{14}$ & 67.01 & 7.62 & 63.44 & 4.18 & $3.95 \times 10^{-11}$ & $3.06 \times 10^{-10}$ & $1.29 \times 10^{-1}$ \\
$3.0 \times 10^{14}$ & 70.14 & 10.75 & 64.84 & 5.91 & $1.64 \times 10^{-10}$ & $9.24 \times 10^{-10}$ & $1.78 \times 10^{-1}$ \\
$4.0 \times 10^{14}$ & 75.37 & 15.98 & 67.16 & 8.82 & $8.07 \times 10^{-10}$ & $3.43 \times 10^{-9}$ & $2.35 \times 10^{-1}$ \\
$5.0 \times 10^{14}$ & 79.69 & 20.30 & 69.07 & 11.23 & $2.04 \times 10^{-9}$ & $7.74 \times 10^{-9}$ & $2.63 \times 10^{-1}$ \\
$6.0 \times 10^{14}$ & 83.41 & 24.02 & 70.71 & 13.31 & $3.87 \times 10^{-9}$ & $1.39 \times 10^{-8}$ & $2.78 \times 10^{-1}$ \\
$7.0 \times 10^{14}$ & 86.69 & 27.30 & 72.15 & 15.15 & $6.24 \times 10^{-9}$ & $2.19 \times 10^{-8}$ & $2.84 \times 10^{-1}$ \\
$8.0 \times 10^{14}$ & 89.63 & 30.24 & 73.43 & 16.81 & $9.08 \times 10^{-9}$ & $3.17 \times 10^{-8}$ & $2.86 \times 10^{-1}$ \\
$9.0 \times 10^{14}$ & 92.31 & 32.92 & 74.60 & 18.32 & $1.24 \times 10^{-8}$ & $4.33 \times 10^{-8}$ & $2.85 \times 10^{-1}$ \\
$1.0 \times 10^{15}$ & 94.77 & 35.38 & 75.67 & 19.71 & $1.60 \times 10^{-8}$ & $5.61 \times 10^{-8}$ & $2.83 \times 10^{-1}$ \\
$1.5 \times 10^{15}$ & 104.88 & 45.49 & 80.05 & 25.44 & $3.90 \times 10^{-8}$ & $1.48 \times 10^{-7}$ & $2.64 \times 10^{-1}$ \\
$2.0 \times 10^{15}$ & 112.70 & 53.31 & 83.43 & 29.88 & $6.78 \times 10^{-8}$ & $2.78 \times 10^{-7}$ & $2.43 \times 10^{-1}$ \\
$2.5 \times 10^{15}$ & 119.17 & 59.78 & 86.21 & 33.57 & $1.01 \times 10^{-7}$ & $4.47 \times 10^{-7}$ & $2.26 \times 10^{-1}$ \\
$2.8 \times 10^{15}$ & 122.59 & 63.20 & 87.67 & 35.52 & $1.22 \times 10^{-7}$ & $5.56 \times 10^{-7}$ & $2.20 \times 10^{-1}$ \\
$3.0 \times 10^{15}$ & 124.72 & 65.33 & 88.59 & 36.74 & $1.38 \times 10^{-7}$ & $6.52 \times 10^{-7}$ & $2.11 \times 10^{-1}$ \\
\hline
\end{tabular}

used. Inserting $\frac{2 \pi}{\hbar} \frac{G_{\mathrm{F}}^{2} C_{\mathrm{V}}^{2}\left(1+3 a^{2}\right)}{\left(2 \pi^{2} \hbar^{3} c^{3}\right)^{2}}=0.018(\mathrm{MeV})^{-5} \mathrm{~s}^{-1}$ and $m_{\mathrm{e}} c^{2}=0.511 \mathrm{MeV}$ into Eq.(8) and Eq.(9), we obtain the values of electron capture rate in superhigh magnetic fields. The calculation results are partly listed below in tabular form. The main results in Table 1 are presented as follows: When the magnetic field $B \sim\left(3.0 \times 10^{15}-1.8 \times 10^{14}\right) \mathrm{G}, \Gamma \sim\left(1.38 \times 10^{-7}-\right.$ $\left.2.43 \times 10^{-13}\right) \mathrm{s}^{-1}$ and $\Gamma^{\prime} \sim\left(6.52 \times 10^{-7}-7.76 \times 10^{-12}\right) \mathrm{s}$ ${ }^{-1}$, respectively. From Table 1 , the values of $\Gamma$ are universally less than those of $\Gamma^{\prime}$, and the ratio of $K=\Gamma / \Gamma^{\prime}$ is about the magnitude of $10^{-1}-10^{-2}$. The possible explanations are as follows:

1. Due to the formation of Landau cylinder in the momentum space or the quantization of Landau levels or, the spherical symmetry in the momentum space is broken by superhigh magnetic fields. The formu- lae of $\rho_{\mathrm{e}}$ and $\rho_{\mathrm{e}}^{\prime}$ are derived in circular cylindrical coordinates and in spherical coordinates, respectively.

2. In the vicinity of the Fermi surface, the electrons with the same energy $E$ could come from different Landau levels because the electrons are degenerate. However, the electrons occupying lower Landau levels cannot be captured even if their energies are higher than $Q$; for higher Landau levels, there still exist some electrons with lower energies $E(E<Q)$ that are not captured. Thus, the values of $\Gamma$ calculated by the expression of $\rho_{\mathrm{e}}$ in superhigh magnetic fields are less than those computed by the expression of $\rho_{\mathrm{e}}^{\prime}$ in non-relativistic magnetic fields.

3. In order to obtain the values of the effective electron capture rates $\Gamma_{\text {eff }}$ in superhigh magnetic fields, we introduce the Landau level effect coefficient $q$ (see Papers 1-2). In our improved model, the following factors: neutron superfluid's restraining effect, the 
numbers of all particles participating in EC reaction, thermal energy loss, energy conversion efficiency and so on, have been considered, so the quantity $q$ must be replaced by two important parameters: $\Lambda$ and $\zeta$ (see Paper 4). Actually, the quantity $q$ includes the effects of $\Lambda$ and $\zeta$. Thus, the values of $K$ is far less than those of $q$ (see Papers 1-2).

From Table 1, we obtain the diagram of $K$ as a function of $B$, shown as in Fig.3. Furthermore, the analytic

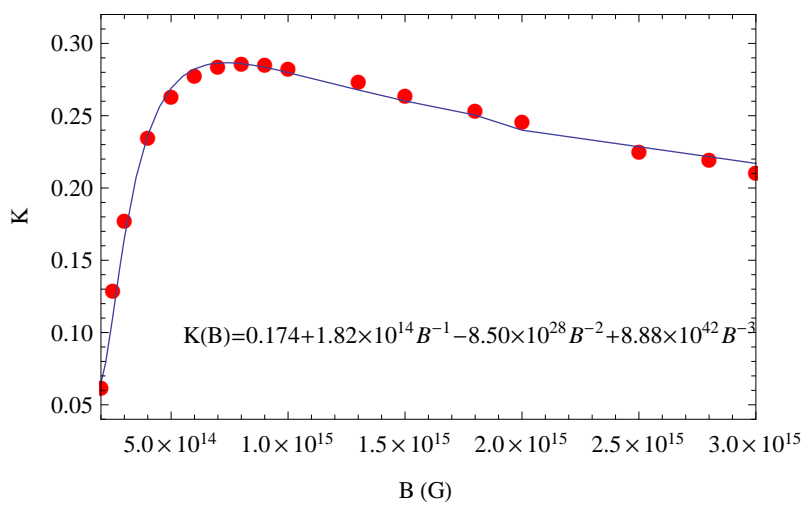

Fig. 3 The diagram of $K$ versus $B$

expression of $K$ and $B$ is obtained by fitting the data of Table 1,

$K(B)=0.174+\frac{1.82 \times 10^{14}}{B}-\frac{8.50 \times 10^{28}}{B^{2}}+\frac{8.88 \times 10^{42}}{B^{3}}$,

From the definition of $K=\Gamma / \Gamma^{\prime}$, it is obvious that the value of $K$ is determined by $B$, and is irrelevant to $T$.

Since the updated expression of $\rho_{\mathrm{e}}$ is utilized, the values of $\Gamma_{\mathrm{e}}$ are universally lower than those of $\Gamma_{\mathrm{e}}^{\prime}$ slightly, however, we cannot differentiating Eq.(8) directly. To the contrary, Eq.(9) is very useful in differential calculations, especially in calculating magnetic fields' evolutionary timescales. In order to obtain a second-order differential equation of $B$ and $t$, we may combing Eq.(8) with Eq.(9). By using Eq.(10), we obtain an approximation relation between $\Gamma$ and $\Gamma^{\prime}$,

$$
\begin{aligned}
& \Gamma=K(B) \Gamma^{\prime}=0.018 K(B) \Lambda(B, T) \\
& \times \int_{Q}^{E_{\mathrm{F}}(\mathrm{e})}\left(E_{\mathrm{e}}^{2}-0.261\right)^{\frac{1}{2}} E_{\mathrm{e}}\left(E_{\mathrm{e}}-Q\right)^{2} d E_{\mathrm{e}} .
\end{aligned}
$$

\section{Magnetic field decay of magnetars in SNRs}

\subsection{Superhigh magnetic fields and their evolutionary timescales}

In order to investigate the whole process of the decay of superhigh magnetic fields, to begin with, let us make two approximations: 1. A magnetar can be treated as a common NS with a total mass of $M=2.8 \times 10^{33} \mathrm{~g}$ (that is about 1.4 times the solar mass) and a radius of $R=10^{6} \mathrm{~cm} ; 2$. The whole electron capture timescale is equal to the decay timescale of superhigh magnetic fields (without consideration of the modified Urca process).

As discussed in Sec.1, if the ${ }^{3} P_{2}$ neutron Cooper pairs are destroyed by the outgoing EC neutrons, both the anisotropic superfluid and superhigh magnetic fields produced by the aligned magnetic moments of the ${ }^{3} P_{2}$ Cooper pairs will disappear gradually. Employing Eq.(10) can allow us to gain a differential equation

$$
\begin{aligned}
& \frac{d \Gamma}{d t} \approx K(B) \frac{d \Gamma^{\prime}}{d t}=0.018 K(B) \Lambda(B, T)\left(E_{\mathrm{F}}(\mathrm{e})-Q\right)^{2} \\
& E_{\mathrm{F}}(\mathrm{e})\left(E_{\mathrm{F}}^{2}(\mathrm{e})-m_{\mathrm{e}}^{2} c^{4}\right)^{\frac{1}{2}} 43.44 \times \frac{1}{4} B^{-\frac{3}{4}} B_{\mathrm{cr}}^{-\frac{1}{4}} \frac{d B}{d t},(12)
\end{aligned}
$$

where $\frac{d(0.018 K(B) \Lambda(B, T))}{d t}$ is ignored because of its too low value $\left(0.018 K(B) \Lambda(B, T) \sim 10^{-17} \sim 10^{-18}(\mathrm{MeV})\right.$ $-5 \mathrm{~s}^{-1}, \frac{d(0.018 K(B) \Lambda(B, T)}{d t} \sim \Delta(0.018 K(B) \Lambda(B, T) \sim$ $\frac{10^{-17} \sim 10^{-18}(\mathrm{MeV})^{-5} \mathrm{~s}^{-1}}{10^{13} \mathrm{~s}} \sim 10^{-30} \sim 10^{-31}(\mathrm{MeV})^{-5} \mathrm{~s}^{-2}$, the integral term $\int_{Q}^{E_{\mathrm{F}}(\mathrm{e})}\left(E_{\mathrm{e}}^{2}-0.261\right)^{\frac{1}{2}} E_{\mathrm{e}}\left(E_{\mathrm{e}}-Q\right)^{2} d E_{\mathrm{e}} \sim$ $10^{10}-10^{6}(\mathrm{MeV})^{5}$, and its time derivative $\sim 10^{-3}-$ $10^{7}(\mathrm{MeV})^{5} \mathrm{~s}^{-1}$ assuming $\left.\Delta t \sim 10^{6} \mathrm{yrs}\right)$. Using binomial expansion theorem, the term $\left(E_{\mathrm{F}}^{2}(\mathrm{e})-m_{\mathrm{e}}^{2} c^{4}\right)^{\frac{1}{2}}$ can be expanded as:

$$
\begin{aligned}
& \left(E_{\mathrm{F}}^{2}(e)-m_{\mathrm{e}}^{2} c^{4}\right)^{\frac{1}{2}}=E_{\mathrm{F}}(e)\left(1-m_{\mathrm{e}}^{2} c^{4} / 2 E_{\mathrm{F}}^{2}(e)\right. \\
& \left.-m_{\mathrm{e}}^{4} c^{8} / 8 E_{\mathrm{F}}^{4}(e)+\cdots\right) \approx 43.44\left(\frac{B}{B_{\mathrm{cr}}}\right)^{\frac{1}{4}} \\
& \times\left(1-542 B^{-\frac{1}{2}}-146932 B^{-1}+\cdots\right) .
\end{aligned}
$$

Since $542 B^{-\frac{1}{2}} \sim 10^{-5}$ and $146932 B^{-1} \sim 10^{-10}$, we will reserve the first term in the bracket of the binomial expansion in the following calculations. Since a normal radio pulsar can be treated as a system of magnetic dipoles, there is an approximation relation of $\mu=\frac{1}{2} B R_{6}^{3}$, where $\mu, B$ and $R_{6}$ are the dipole magnetic moment, the dipole magnetic field strength and the radius of the star in units of $10^{6} \mathrm{~cm}$, respectively (Shapiro \& Teukolsky 1983). Like normal radio pulsars, a magnetar can be seen as a magnetic dipole system, the above approximation relation is also hold in a magnetar. Assuming that one outgoing EC neutron 
can destroy one ${ }^{3} P_{2}$ Cooper pair (see Appendix A), the decay rates of magnetic fields of magnetars can be estimated as

$\frac{d B}{d t}=\frac{2}{R_{6}^{3}} \frac{d \mu}{d t}=\frac{2}{R_{6}^{3}}\left(-\Gamma 2 \mu_{\mathrm{n}} n_{\mathrm{e}} V\left({ }^{3} P_{2}\right)\right)$,

where $V\left({ }^{3} P_{2}\right)$ denotes the volume of the ${ }^{3} P_{2}$ anisotropic neutron superfluid, $V\left({ }^{3} P_{2}\right)=\frac{4}{3} \pi R_{5}^{3} \mathrm{~cm}^{3}, R_{5}=10^{5} \mathrm{~cm}$, and $n_{\mathrm{p}}=n_{\mathrm{e}}=9.6 \times 10^{35} \mathrm{~cm}^{-3}$ setting $\rho=\rho_{0}$. Since $\Gamma$ in this paper represents the effective electron capture rate, Eq.(14) deviates greatly from Eq.(11) in Paper 2, though they are exactly like. Be note, in the interior of a NS, the processes of EC and $\beta$-decay exist at the same time, which is required by electric neutrality, the depleted protons/electrons are recycled for many times, so the alteration of $Y_{\mathrm{p}} / Y_{\mathrm{e}}$ could be very small. From Eq.(14), we get

$\frac{d \Gamma}{d t}=\frac{-R_{6}^{3}}{4 \mu_{\mathrm{n}} n_{\mathrm{e}} V\left({ }^{3} P_{2}\right)} \frac{d^{2} B}{d t^{2}}$,

where $\mu_{n}=0.966 \times 10^{-23} \mathrm{erg} \mathrm{G}^{-1}$ is the absolute value of the neutron abnormal magnetic moment. Combining Eq.(12) with Eq.(15) and eliminating $\Gamma$ yields a secondorder differential equation:

$$
\begin{aligned}
& \frac{d^{2} B}{d t^{2}}+4.8002 \times 10^{24}\left(2.60388 \times 10^{14}+1.98233\right. \\
& \left.\times 10^{-30} B\right)\left(0.174+1.82 \times 10^{14} B^{-1}-8.50 \times 10^{28}\right. \\
& \left.B^{-2}+8.88 \times 10^{42} B^{-3}\right)\left(1.98 \times 10^{-25} B^{\frac{1}{4}}-1.394\right. \\
& \left.\times 10^{-21}+2.458 \times 10^{-18} B^{-\frac{1}{4}}\right) \frac{d B}{d t}=0,
\end{aligned}
$$

where $T_{0}=2.6 \times 10^{8} \mathrm{~K}$ is used. For the purpose of calculating the whole electron capture time $t$, we can treat this second-order differential equation as follows: Firstly, decreasing the order of Eq.(16) gives a firstorder differential equation

$$
\begin{aligned}
& \frac{d B}{d t}=-\left(-1.2125 \times 10^{36} B^{\frac{-9}{4}}+7.73615 \times 10^{32}\right. \\
& B^{-2}-1.2558 \times 10^{29} B^{\frac{-7}{4}}+2.0725 \times 10^{22} B^{\frac{-5}{4}}- \\
& 1.46924 \times 10^{19} B^{1}+2.78251 \times 10^{15} B^{\frac{-3}{4}}-2.1571 \\
& \times 10^{8} B^{\frac{-1}{4}}+17.3762 B^{\frac{1}{4}}+7.69529 \times 10^{-8} B^{\frac{3}{4}} \\
& -3.27316 \times 10^{-11} B-30.5838 \log [B]+3.7193 \\
& -1.15403 \times \times 10^{-15} B^{\frac{5}{4}}+2.32557 \times 10^{-24} B^{\frac{7}{4}} \\
& \left.10^{-27} B^{2}+1.45703 \times 10^{-31} B^{\frac{9}{4}}\right)+C ;
\end{aligned}
$$

Secondly, inserting the boundary condition: $d B / d t=0$ when $B=B_{\text {th }}$, into Eq.(17) determines the constant of integral $C=-992890.0$; Thirdly, integrating over $B$ gives a general expression of $t$

$$
\begin{aligned}
& t=\int_{B_{\mathrm{i}}}^{B_{\mathrm{f}}}-\left(-1.2125 \times 10^{36} B^{\frac{-9}{4}}+7.73615 \times 10^{32}\right. \\
& B^{-2}-1.2558 \times 10^{29} B^{\frac{-7}{4}}+2.0725 \times 10^{22} B^{\frac{-5}{4}}- \\
& 1.46924 \times 10^{19} B^{1}+2.78251 \times 10^{15} B^{\frac{-3}{4}}-2.1571 \\
& \times 10^{8} B^{\frac{-1}{4}}+17.3762 B^{\frac{1}{4}}+.69529 \times 10^{-8} B^{\frac{3}{4}} \\
& -3.27316 \times 10^{-11} B-30.5838 \log [B]+3.7193 \times \\
& 10^{-15} B^{\frac{5}{4}}+2.32557 \times 10^{-24} B^{\frac{7}{4}}-1.15403 \times 10^{-27} \\
& \left.B^{2}+1.45703 \times 10^{-31} B^{\frac{9}{4}}+992890.0\right)^{-1} d B ; \quad(18)
\end{aligned}
$$

Finally, using integral transform $B^{\frac{1}{4}} \rightarrow x$ and $d B \rightarrow$ $4 x^{3} d x$ gives the final expression of $t$

$$
\begin{aligned}
& t=\int_{B_{\mathrm{f}}^{0.25}}^{B_{\mathrm{i}}^{0.25}}\left(-1.2125 \times 10^{36} x^{-9}+7.73615 x^{-8}-\right. \\
& 1.2558 \times 10^{29} x^{-7}+2.0725 \times 10^{22} x^{-5}-1.46924 \\
& \times 10^{19} x^{4}+2.78251 \times 10^{15} x^{-3}-2.1571 \\
& \times 10^{8} x^{-1}+17.3762 x+7.69529 \times 10^{-8} x^{3} \\
& -3.27316 \times 10^{-11} x^{4}-30.5838 \log \left[x^{4}\right]+3.7193 \times \\
& 10^{-15} x^{5}+2.32557 \times 10^{-24} x^{7}-1.15403 \times 10^{-27} \\
& \left.x^{8}+1.45703 \times 10^{-31} x^{9}+992890.0\right)^{-1} 4 x^{3} d x, \quad(19)
\end{aligned}
$$

where $x \geq B_{\mathrm{th}}^{0.25}=3524.05$. In order to investigate the characteristics of Eq.(19), we introduce a variable $F(x)$ to denote the integrated function, $F(x)=(-1.2125 \times$ $10^{36} x^{-9}+7.73615 \times 10^{32} x^{-8}-1.2558 \times 10^{29} x^{-7}+$ $2.0725 \times 10^{22} x^{-5}-1.46924 \times 10^{19} x^{-4}+2.78251 \times$ $10^{15} x^{-3}-2.1571 \times 10^{8} x^{-1}+17.3762 x+7.69529 \times$ $10^{-8} x^{3}-3.27316 \times 10^{-11} x^{4}-30.5838 \log \left[x^{4}\right]+3.7193 \times$ $10^{-15} x^{5}+2.32557 \times 10^{-24} x^{7}-1.15403 \times 10^{-27} x^{8}+$ $\left.1.45703 \times 10^{-31} x^{9}+992890.0\right)^{-1} 4 x^{3}$, and make a schematic diagram of $F(x)$ as a function of $x$, shown as in Fig.4. From Fig.4, in the integral interval of

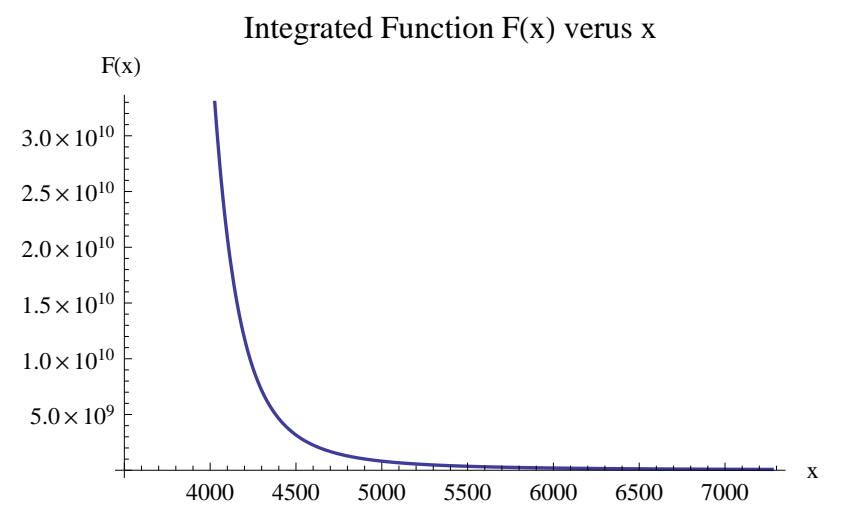

Fig. 4 The diagram of $F(x)$ versus $x$ 
[( $\left.\left.x_{1} \sim x_{2}\right), x_{1} \leq x_{2}\right]$, the function $F(x)$ is convergent, and hence is integrable, where $x=3524.05$ is a singularity of $F(x)$. By solving Eq.(19), we gain the whole electron capture time (or the superhigh magnetic field's decay timescale), $t \approx 9.2947 \times 10^{13} \mathrm{~s}=2.9507 \times 10^{6}$ yrs when $B_{\mathrm{i}}=3.0 \times 10^{15} \mathrm{G}$ and $B_{\mathrm{f}}=B_{\mathrm{th}}$; in the same way, if $B_{\mathrm{i}}=3.0 \times 10^{15} \mathrm{G}$ and $B_{\mathrm{f}}=4.0 \times 10^{14} \mathrm{G}$, $t \approx 1.4737 \times 10^{12} \mathrm{~s}=4.6785 \times 10^{4}$ yrs, corresponding to $L_{\mathrm{X}} \sim\left(10^{37} \sim 10^{34}\right)$ erg s${ }^{-1}$. Furthermore, the fitting curves of $\log _{10} B$ versus $\log _{10} t$ for different magnetic field ranges are shown in Fig. 5.

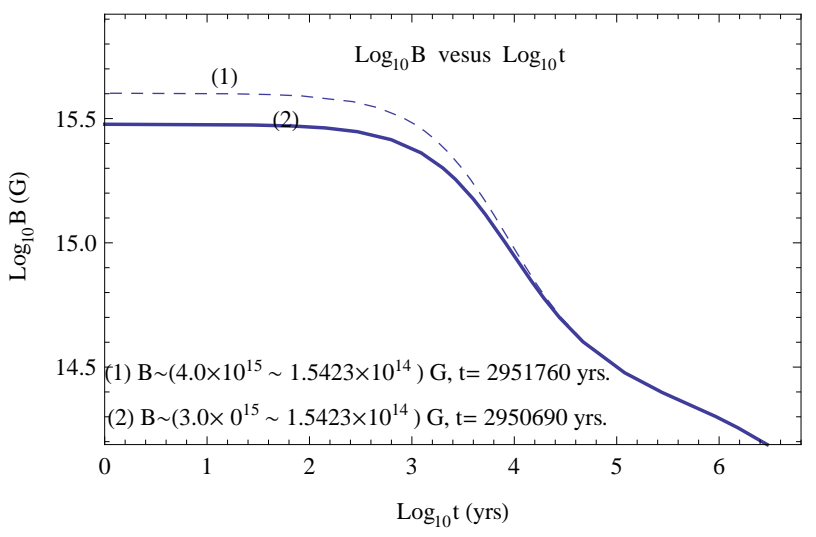

Fig. 5 Superhigh magnetic field $B$ as a function of time $t$.

From Fig.5, the magnetic field decreases with increasing time obviously. Customarily, $B=3.0 \times 10^{15}$ $\mathrm{G}$ is assumed to be the possible initial magnetic field, while $B=4.0 \times 10^{15} \mathrm{G}$ is assumed to be the upper limit of magnetic field of a magnetar by some authors (e.g., recent papers of Peng \& Tong 2007, 2009, and Gao et al 2011). However, the difference between the evolution timescales of these two fields is only $\Delta t \simeq 1070 \mathrm{yrs}$, obtained from Eq.(19) (as shown in Fig.5), which implies a substantial positive correlation between the magnetic field and its decay rate.

Since the initial value of internal temperature $T$ is far higher than its current value for a magnetar, we arbitrarily assume $T_{0} \sim 2.75 \times 10^{8}-1.75 \times 10^{8} \mathrm{~K}$ within a permitted and plausible temperature range under our magnetar model. Repeating all the calculations above gives the relation of $t$ and $T_{0}$, shown as in Fig.6.

From Fig.6, it is apparent that the lower $T_{0}$, the higher $t$ if $B_{0}$ is invariable. When $B_{0}=3.0 \times 10^{15}$ $\mathrm{G}, T_{0} \sim 2.75 \times 10^{8}-1.75 \times 10^{8} \mathrm{~K}$, then $t \sim 2.098 \times$ $10^{6}-4.077 \times 10^{7}$ yrs.

\subsection{The initial internal fields of magnetar progenitors}

Although we have presented a reasonable explanation for the origin of superhigh magnetic fields in the previous work (Peng \& Tong 2007; Peng \& Tong 2009),

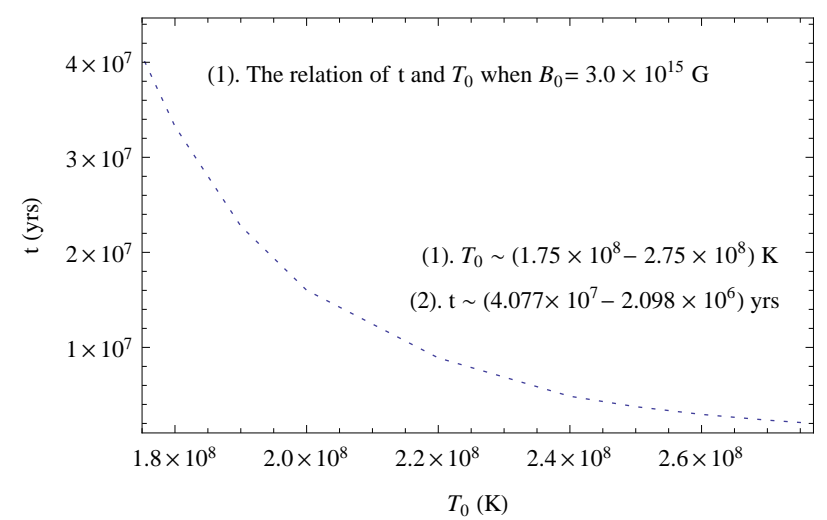

Fig. 6 The magnetic field evolution timescale $t$ as a function of time $T_{0}$.

many issues concerning magnetars remain unsolved. So far, the magnetic fields of magnetars obtained from the observations are just their surface dipolar magnetic fields $B_{\text {dip }}$, assuming a simple magnetic dipole spindown model. What is the relationship between the surface dipolar magnetic field and the internal magnetic field in a magnetar? How strong is the initial internal magnetic field $B_{\mathrm{i}}$ of a magnetar progenitor? How long will such an intense field $\left(B_{\mathrm{i}}\right)$ continue to decay? What will eventually happen in the interior of a magnetar when $B$ drops below $B_{\text {th }}$ ? All these questions are all very basic, and remain open.

In this part, motivated by SNR associations, we try to carry out the studies of $B_{\mathrm{i}}$ for magnetar progenitors. Observations indicate that SNRs have been expanding, and interacting with their surroundings since the supernova explosions. Therefore, the ages of SNRs may be computed by modeling their morphologies at the current epoch. The true ages of magnetars obtained from the ages of their SNRs are independent of the stars' properties, and thus basically unbiased even if AXPs or SGRs are strange stars (Zhang et al. 2000; $\mathrm{Xu}$ et al. 2006). Table 2 shows the data on 9 claimed magnetar-supernova remnant associations, which are cited from McGillAXP/SGR online catalog updated except for SGR 1806-20 and SGR 1900+14. As is known to us, all the known SNRs associated with common radio pulsars are very young, $t_{\mathrm{SNR}} \ll 10^{6} \mathrm{yrs}$. From Table 2 , the ages of all the SNRs are not more than 10,000 yrs, which implies that the associated magnetars are more younger, compared with common radio pulsars. Perhaps these magnetars born with different physical properties (eg., the equations of state, magnetic fields inner temperatures, and so on) have experienced evolutionary routes that differ from those of common radio 
Table 2 The calculated values of the initial internal magnetic fields of magnetar progenitors

\begin{tabular}{crrrrrrr}
\hline Source & $P$ & $\dot{P}$ & $B_{\text {dip }}$ & SNR & $t_{\text {SNR }}$ & $B_{\mathrm{i}}^{a}$ & Ref $^{d}$ \\
\hline SGR 0526-66 & 8.0544 & 3.8 & 5.6 & N49 & 5.0 & 5.06 & {$[1,2]$} \\
SGR 1806-20 & 7.6022 & 75 & 24 & G10.0-0.3 & $<10^{\ddagger}$ & $\leq 29.3$ & {$[3,4]$} \\
SGR 1627-41 & 2.5946 & 1.9 & 2.20 & G33.70-01 & 5.0 & 2.204 & {$[5,6]$} \\
SGR 1900+14 & 5.1998 & 9.2 & 7.0 & G42.8+0.6 & $<10^{\boldsymbol{*}}$ & $<13.71$ & {$[7,8]$} \\
1E 2259+586 & 6.9789 & 0.048 & 0.59 & CTP109 & $\sim 10$ & $\sim 1.5435$ & {$[9,10]$} \\
1E 1841-045 & 11.7829 & 3.93 & 6.9 & Kes73 & 2 & 7.497 & {$[11,12]$} \\
1E 1547.0-5408 & 2.318 & 2.318 & 2.2 & G327.24-0.13 & $<1.4$ & $<2.202$ & {$[13,14]$} \\
CXOU J171405.7 & 3.82535 & 6.40 & 5.0 & CTB37B & 4.9 & 5.509 & {$[15-17]$} \\
AX J1845-0258 & 6.97127 & No & No & G29.6+0.1 & $<8$ & $\leq 40^{b}$ & {$[18,19]$} \\
\hline
\end{tabular}

Note: The units of period $P$, period derivative $\dot{P}$, the surface dipolar magnetic field $B_{\text {dip }}$ SNR's age $t_{\text {SNR }}$ and the initial internal magnetic field $B_{\mathrm{i}}$ are s, $10^{-11} \mathrm{~s} \mathrm{~s}^{-1}, 10^{14} \mathrm{G}, 10^{3}$ yrs and $10^{14} \mathrm{G}$, respectively. The sign 'a' denotes: The values of the initial internal magnetic fields of magnetar progenitors $B_{\mathrm{i}}$ are gained by using Eq.(18) and $t \simeq t_{\mathrm{SNR}}$. The sign ' $\mathrm{b}$ ' denotes: Since AXP $1 \mathrm{E}$ $2259+586$ could be associated with accretion (see Paper 4 ), and its present value of $B_{\text {dip }}$ is far less than $B_{\text {th }}$, its maximum value of $B_{\mathrm{i}}$ has to be estimated under our model. The sign 'c' denotes: Since some important parameters (eg., $\dot{P}, B_{\mathrm{dip}}$, the soft X-ray luminosity $L_{\mathrm{X}}$, and so on) of magnetar candidate AX J1845-0258 are uncertain, its maximum value of $B_{\mathrm{i}}$ has to be estimated under our model. The sign 'd' denotes:[1]:(Kulkarni et al. 2003); [2]:(Klose et al.2004); [3]:(Kulkarni \& Frail 1993); [4]:(Marsden et al. 2001); [5]: (Corbel et al. 1999); [6]: Wachter et al. 2004); [7]: (Hurley et al.|1999); [8]: (Mazets et al.|1999) [9]: (Green 1989); [10]:(Rho \& Petre 1997); [11]: (Sanbonmatsu \& Herfand 1992); [12]: (Vasisht \& Gotthelf 1997); [13]:(Camilo et al. 2007); [14]: (Gelfand \& Gaensler 2007); [15]: (Aharonian et al. 2008); [16]:(Halpern \& Gotthelf 2010); [17]: (Horvath \& Allen 2011); [18]: (Gaensler et al. 1999); [19]: (Vasisht et al. 2000). The sign ' $\dagger$ ' denotes: This candidate is unconfirmed. The sign ' $\ddagger$ ' denotes: cited from Kulkani \& Frail(1993) and Marsden et al.(2001). The sign '\$' denotes: cited from Hurley et al.(1999). All primitive data are from McGillAXP/SGR online catalog of 2 March 2012(http://www.physics. mcgill.ca/ pulsar/magnetar/ main.html) and references cataloged.

pulsars. For the sake of computing conveniently, we assume a simple magnetic dipole spin-down model, then the current magnetic field inside a magnetar is about its surface dipole field, i.e., $B_{\mathrm{f}} \simeq B_{\text {dip }}$ under this assumption. The field decay timescale of a magnetar equals approximately to the age of its SNR, i.e., $t \simeq t_{\mathrm{SNR}}$, base on the results of ZX2011. Combining Eq.(19) with Table 2 gives the values of $B_{\mathrm{i}}$ for magnetar progenitors. The calculated results show that the values of $B_{\mathrm{i}}$ are concentrated primarily between $2.0 \times 10^{14} \mathrm{G}$ and $2.93 \times 10^{15} \mathrm{G}$ when $T_{0} \sim 2.60 \times 10^{8} \mathrm{~K}$. If $T_{0} \sim 2.75 \times 10^{8}-1.75 \times 10^{8} \mathrm{~K}$, there still be $B_{\mathrm{i}} \sim\left(10^{14}-10^{15}\right) \mathrm{G}$, not exceeding the upper limit of magnetic field of $4.0 \times 10^{15} \mathrm{G}$. The calculated results of $B_{\mathrm{i}}$ for magnetar progenitors illustrate that our magnetar model is consistent theoretically.

\subsection{The spin-down ages of magnetars and the ages of their SNRs}

As pointed out in Sec.1, the NS's spin-down age, also called the characteristic age, can be generally expressed as, $t_{\text {Spin }}=P /(n-1) \dot{P}$. In all kinds of catalog on pulsars, the spin-down ages of pulsars are usually evaluated by a simple magnetic dipole spin-down model, $t_{\text {Spin }}=P / 2 \dot{P}(n=3)$. In ZX2011, authors found that there is a strong and significant positive correlation between $\log _{10}(\operatorname{SNR}($ Age $) /$ Spindown(Age)) and $\log _{10} B$ via statistical analysis of radio pulsars associated with SNRs. They argued that, as NSs get older, their spin periods become longer duo to their spin-down torques, the decay of magnetic fields cause $\dot{P}$ to be far less than the mean values of $\dot{P}$ in history, their characteristic ages, inferred from parameters $P$ and $\dot{P}$ at the current epoch, will be larger than their real ages, denoted as $t_{\text {Real }}$. In a word, it's the dipolar magnetic field decay that plays a significant role in making a NS look older than it really is.

In this part, we investigate the spin-down ages of magnetars and the ages of their SNRs, and made a diagram of $\log _{10}(\mathrm{SNR}$ (Age)/Spindown(Age)) versus $\log _{10} B$.

From Fig.7, an obvious correlation has been proved between $\log _{10} B$ and $\log _{10}(\mathrm{SNR}$ (Age)/Spindown(Age) for magnetars associated with SNRs. It is worthwhile to note that all AXPs associated with SNRs are on the left of the dashed line whereas all SGRs associated with SNRs are on the left of the line. The causes of this will be discussed at length in the following.

Since a magnetar can be seen as a magnetic dipole system, the above suggestion that the dipolar magnetic field decay plays a significant role in making a NS look older' is also applicable to magnetars. For all the AXPs (including candidates) with dipole magnetic fields $B<7.0 \times 10^{14} \mathrm{G}$, there have been no super-bursts or giant flares, at which huge energies $\left(\sim 10^{43}-10^{47} \mathrm{erg}\right)$ are suddenly released. This suggests that all AXPs could have experienced relatively 'normal' decay of their dipole magnetic fields compared 


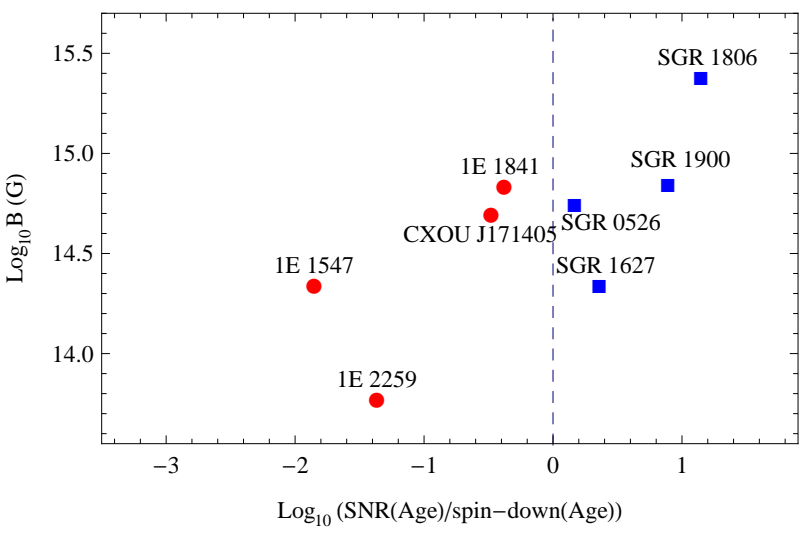

Fig. $7 \log _{10}(\operatorname{SNR}($ Age $) /$ Spindown(Age) $)$ versus $\log _{10} B$. The range of $B$ is $\left(1.5423 \times 10^{14} \sim 3.0 \times 10^{15}\right) \mathrm{G}$. The dashed line corresponds to $t_{\mathrm{SNR}}=t_{\mathrm{Spin}}$.

with SGRs associated with SNRs, and thus have lower braking indexes, $n<3$.

In previous works, many authors proposed various models to explain why the observed braking index $n<3$, eg., neutrino and photon radiation coming from superfluid neutrons may brake the pulsars (Peng et al. 1982); both magnetic dipole radiation and the propeller torque applied by the debris disk may cause spindown of pulsars (Alpar et al. 2001; Menou et al. 2001); the combination of dipole radiation and the unipolar generator may cause $n$ decrease greatly $\mathrm{Xu} \&$ Qiao 2001; Wu et al 2003); a variation of the torque function is important attribution for low braking index (Allen \& Horvath 1997); additional torques due to accretion may cause $n$ decrease Menou et al. 2001; Chen \& Li 2006); $n<3$ may be due to the decay of magnetic field strengthes(Blandford \& Romani 1988; Lin \& Zhang 2004; Chen \& Li 2006; Zhang \& Xie 2011), and so on. As we know, magnetars are high magnetized NSs. They universally possess very strong surface dipole magnetic fields $\left(\sim 10^{14}-10^{15} \mathrm{G}\right)$ with rare exceptions (eg., SGR 0418+5729, 1E 2259+586 and unconfirmed candidate Swift J1822.3-1606). Thus, we favor the braking model with changing magnetic field strengthes, ie., the decay of magnetic field leads to $n<3$ for magnetars. In our magnetar model, the lower braking indices $n(n<3)$ of AXPs are supposed to be correlated with the dipole magnetic fields $B$ and their decay rates $d B / d t$. In order to validate this assumption,, we investigate the phenomenon of $n<3$ for neutron stars (including common radio pulsars and magnetars) under pure magnetic dipole spin-down model, theoretically.

As we know, the spin frequency $\Omega$ of pulsars decreases with time, and the time derivative of $\Omega$ is pro- portional to some power of $\Omega$,

$I \dot{\Omega}=-K \Omega^{n}$,

where $K=2 B^{2} R^{6} \sin ^{2} \theta / 3 c^{2}, \theta$ is the inclination of the magnetic axis with respect to the rotation axis; $B, R I$ are the surface magnetic field strength, the radius, and the momentum of inertia of the pulsar, respectively; $c$ is the velocity of light(?). The braking index $n$ of a pulsar can be a measured by differentiating Eq.(20),

$n=\frac{\ddot{\Omega} \Omega}{\dot{\Omega}^{2}}$,

where $\ddot{\Omega}$ is the second order time derivative of $\Omega$. In the model that assumes spin-down is due to pure magnetodipole radiation with a constant magnetic field, we obtain the ideal values of $n$ in Eqs.(20-21), $n=3$ (?Blandford \& Romani 1988; Menou et al. 2001; Chen \& Li 2006; Zhang \& Xie 2011). As mentioned in Sec.1, the observed values of $n$ from Eq.(21) always deviate from 3 expected for pure magnetodipole radiation model (only in this case, $t_{\mathrm{Spin}}=t_{\mathrm{SNR}}$ ). With respect to the case of $n<3$, the main and possible causes have been provided, as listed above. In this paper, we will simply discuss the error(or deviation) of $n$ caused by the derivation of Eq.(21) itself. From the deduction above, Eq.(21) is obtained by differentiating Eq.(20), assuming $I$ and $K$ are constant. As a result, $n$ is mainly determined by $\Omega$ (or spin period $P, P=2 \pi / \Omega$ ) and its time derivatives, and is irrelevant to the other quantities. Actually, it's possible that the quantities of $B, \theta, I, R$ and $\Omega$ change in varying degrees, and changes of $B, \theta$, $I$ and/or $R$ with $\Omega$ will induce a braking index $n \neq 3$. Hence, a modification of Eq.(21) is necessary. Keeping the quantities of $\theta, I$ and $R$ unchanged (the variances of these quantities are small usually), we re-differentiate Eq.(20) under pure magnetic dipole spin-down model, and get

$\ddot{\Omega}=\frac{d \dot{\Omega}}{d t}=\frac{-2 R^{6} \sin ^{2} \theta}{3 c^{2} I}\left(2 B \dot{B} \Omega^{3}+3 B^{2} \Omega^{2} \dot{\Omega}\right)$,

Inserting Eq.(20) and Eq.(22) into Eq.(21), we have

$$
\begin{aligned}
& n=\frac{-2 R^{6} \sin ^{2} \theta}{3 c^{2} I}\left(\frac{2 B \dot{B} \Omega^{4}}{\dot{\Omega}^{2}}+\frac{3 B^{2} \Omega^{3} \dot{\Omega}}{\dot{\Omega}^{2}}\right) \\
& =\frac{-2 R^{6} \sin ^{2} \theta}{3 c^{2} I}\left(\frac{9 c^{4} I^{2} \dot{B}}{2 R^{12} \sin ^{4} \theta \Omega^{2} B^{3}}-\frac{9 c^{2} I}{2 R^{6} \sin ^{2} \theta}\right) \\
& =3-\frac{3 c^{2} I \dot{B}}{R^{6} \sin ^{2} \theta \Omega^{2} B^{3}}=3-\frac{3 c^{2} P^{2} I}{4 \pi^{2} R^{6} \sin ^{2} \theta} \frac{\dot{B}}{B^{3}} \\
& =3-\frac{3 c^{2} P^{2} I}{4 \pi^{2} R^{6} \sin ^{2} \theta} \frac{|d B / d t|}{B^{3}},
\end{aligned}
$$

where $\dot{B}$ denotes the absolute value of decay rate of dipole magnetic field without considering any other 
torque (ie., the dipole magnetic field normally decays). We can estimate the magnitude of the second term in Eq.(23) as following: for a common radio pulsar, $I \sim 10^{45} \mathrm{~g} \mathrm{~cm}^{2}, \sin ^{2} \theta \sim 1, R \sim 10^{6} \mathrm{~cm}, P \sim 1 \mathrm{~s}$, $c=3.0 \times 10^{10} \mathrm{~m} \mathrm{~s}^{-1}, \frac{\dot{B}}{B^{3}} \sim \frac{|d B / d t|}{B^{3}} \sim \frac{|\Delta B / \Delta t|}{B^{3}} \sim$ $\frac{1}{B^{2} t} \sim 10^{-38}-10^{-39} \mathrm{G}^{-2} \mathrm{~s}^{-1}\left(\Delta B \sim B \sim 10^{12} \mathrm{G}\right.$, $\left.\Delta t \sim t \sim 10^{14}-10^{15} \mathrm{~s}\right), \frac{3 c^{2} P^{2} I}{4 \pi^{2} R^{6} \sin ^{2} \theta} \frac{\dot{B}}{B^{3}} \sim 10^{-8}-10^{-9}$; for a canonic magnetar, $P \sim 10 \mathrm{~s}, \frac{\dot{B}}{B^{3}} \sim \frac{1}{B^{2} t} \sim$ $10^{-41}-10^{-43} \mathrm{G}^{-2} \mathrm{~s}^{-1}\left(\Delta B \sim B \sim 10^{14}-10^{15}\right.$, $\left.\Delta t \sim t \sim 10^{13} \mathrm{~s}\right), \frac{3 c^{2} P^{2} I}{4 \pi^{2} R^{6} \sin ^{2} \theta} \frac{\dot{B}}{B^{3}} \sim 10^{-9}-10^{-10}$, therefore, when all the quantities of $\theta, I, R$ and $P$ (or $\Omega$ ) remain unchanged, and the dipole magnetic field normally decays, $n \approx 3$, in the ideal situation of $\dot{B}=0$, $n=3$. However, if there are substantial changes of $\theta$, $I, R$ and $P$ (at least one quanity varies), the effects of $\frac{\dot{B}}{B^{3}}$ on $n$ cannot be ignored. Under pure magnetic dipole spin-down model, we produce the diagrams of $d B / d t-B$ and $\frac{\dot{B}}{B^{3}}-B$ by using the method of curve fitting, shown as in Fig.8.

Fig.8 is composed of four sub-figures. The fitted curves in Fig.8 a and Fig.8 b are obtained from Eq.(17). Fig. $8 a$ shows that $\frac{d B}{d t}$ decreases with decreasing $B$ significantly in the directed EC process. In Fig.8 $b, \frac{\dot{B}}{B^{3}}$ increases with decreasing $B$ when $B \sim 3.0 \times 10^{15} \sim$ $(4-5) \times 10^{14} \mathrm{G}$, because $B^{3}$ decreases faster than $\dot{B}$, whereas $\frac{\dot{B}}{B^{3}}$ decreases with decreasing $B$ when $B \sim$ $(4-5) \times 10^{14} \sim 1.5423 \times 10^{14} \mathrm{G}$, and $\frac{\dot{B}}{B^{3}}=0$ when $B=B_{\text {th }}$ because the directed EC reaction ceases. It's worth noting that the modified EC reaction always proceeds in the interior of a neutron star with any magnetic field strength, if the directed EC reaction exists, the modified EC reaction can be ignored (See Paper 1 and Paper 2). If the modified EC reaction dominates, the total magnetic decay rate $\frac{d B}{d t}($ or $\dot{B}$ ) may be determined by many other factors, eg., Ohmic decay, ambipolar diffusion and Hall drift (Goldreich \& Reisenegger 1992; Rheinhardt \& Geppert 2003; Pons et al. 2006). However, we mainly focus the relation of $\frac{\dot{B}}{B^{3}}$ with $B$, rather than a specified way of magnetic field when the modified EC reaction dominates. Using the method of dimensional analysis, we produce the schematic diagrams of $\frac{\dot{B}}{B^{3}}$ as a function $B$ if the magnetic field decay timescale $t$ is given, shown as in Fig. 8 c. The curves in Fig. $8 d$ are produced by the superposition of the curves in Fig. $8 b$ and Fig. $8 c$ in a wide range of $B \sim 3.0 \times 10^{15} \sim 10^{12} \mathrm{G}$. From Fig. $8 d$, the total change trend of $\frac{\dot{B}}{B^{3}}$ is that $\frac{\dot{B}}{B^{3}}$ increases with decreasing $B$. Observations show that for most pulsars, their dipole magnetic fields decay slowly during their lifetimes, and their observed braking indices $n$ are smaller than 3 . For young pulsars including AXPs, an obvious correlation has been proved between $n<3$ and the dipole magnetic fields $B$ as well as their decay rates $d B / d t$, which can be easily seen from the combination of Fig.8 with Eq.(23).

Among the known 12 AXPs (9 confirmed, 3 candidates), 1E $2259+586$ has the weakest dipole magnetic field, $B=5.9 \times 10^{14} \mathrm{G}$, the shortest period derivative, $\dot{P}=4.843 \times 10^{-13} \mathrm{~s} \mathrm{~s}^{-1}$, and the longest spindown or characteristic age, $t_{\text {Spin }}=230 \mathrm{kyr}$. All of these date suggests that the value of $n$ of $1 \mathrm{E} 2259+586$ is less than the ideal value of $n=3$. For $1 \mathrm{E} 2259+586$, its soft X-ray emission could be associated with accretion (White \& Marshall 1984; van Paradijs et al. 1995), which is beyond of our model, and the direct EC reaction ceases due to the weaker field ( $B \ll B_{\text {th }}$ ), however the modified EC reaction still occurs, from which weaker X-ray and weaker neutrino flux are produced. For this source, the weakest dipole magnetic field $B$ and super-low rates of decay of $B$ via the modified EC reaction may be the major causes that contribute to $n<3$.

In the above parts, we explain why all AXPs associated with SNRs appear older than they are. The reason why all SGRs associated with SNRs appear younger than their real ages is studied in the follows. Unlike AXPs, all SGRs can emit short bursts in the hard Xray/soft gamma-ray range with $E \sim 10^{39}-10^{41}$ erg (Mereghetti 2008). Furthermore, giant flares and intermediate flares (or giant outbursts) were detected in SGRs associated with SNRs. Table 3 reports the details of giant/intermediate flares from these four SGRs associated with SNRs. In addition, other intermediate flares occurred in SGR $1900+14$ on August 29, 1998 (Ibrahim et al. 2001), and on April 28, 2001 (Lenters et al. 2003). These two intermediate flares are no longer listed in Table 3 because of their relatively lower energies $\sim 10^{39}-10^{41} \mathrm{erg}$. The data in Table 3 implies a significant correlation between giant/intermediate flares and $t_{\mathrm{SNR}}>t_{\mathrm{Spin}}$ for SGRs. The decay of diploe magnetic fields and giant/intermediate flares can conjointly affect spinning behaviors of SGRs associated with SNRs. However, we suppose that giant/ intermediate flares make SGRs look younger than they are. Now, an explanation of $t_{\mathrm{SNR}}>t_{\mathrm{Spin}}$ for SGRs in the context of the star-quake model of magnetars (Thompson et al. 2002) is presented. The details are as follows:

Giant flares/bursts could be motivated by a largescale fracture of the crust, driven by magnetic stresses; the sudden crust's cracking sets the whole magnetar 'quaking', which results in a significant change in configuration of the dipole magnetic field (including magnetic field strength, magnetic field decay rate, the angle between magnetic axis and spin axis, moment of inertia, magnetic moment and so on). Such a change in configuration of the dipole magnetic field could give rise to 


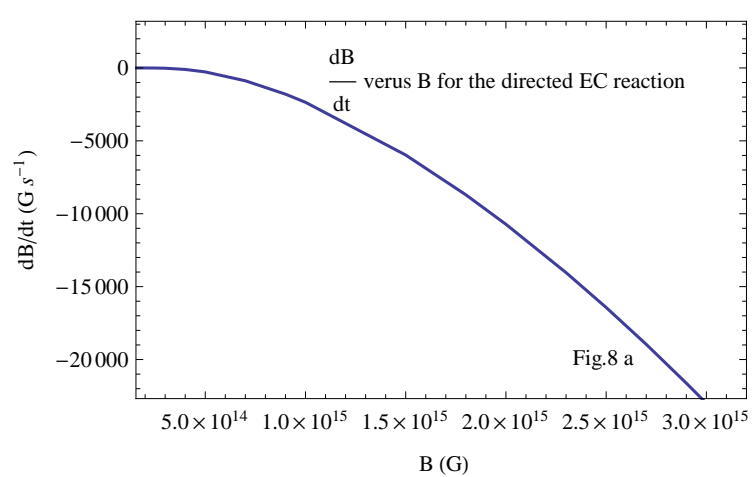

(a)

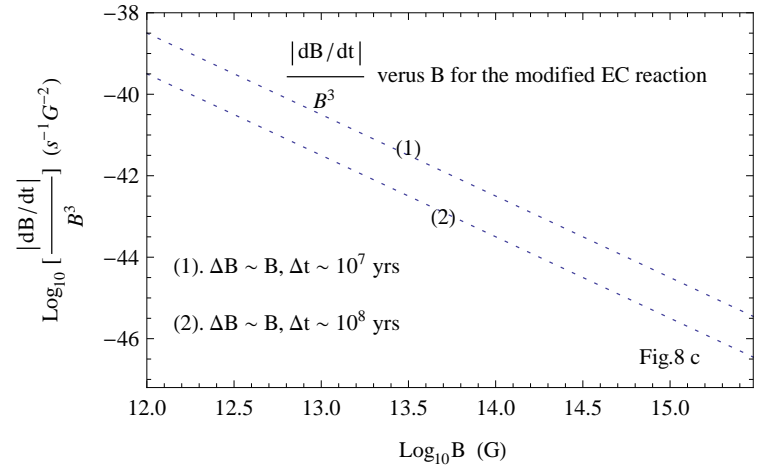

(c)

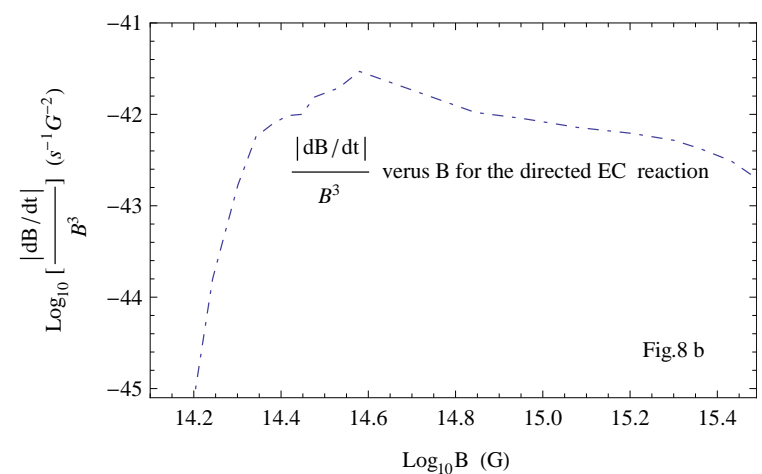

(b)

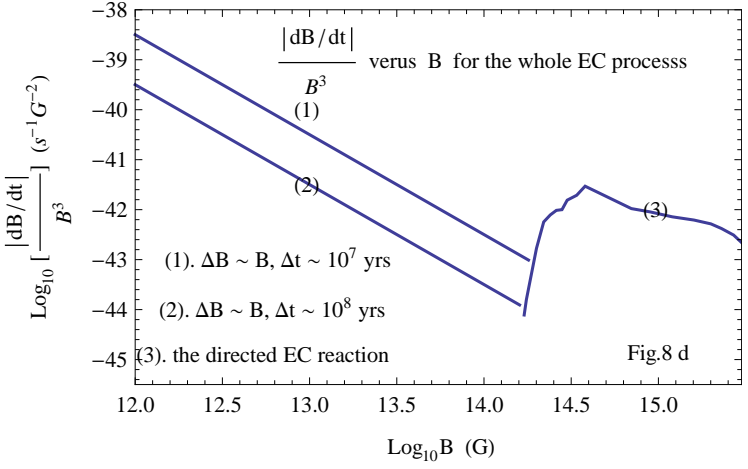

(d)

Fig. 8 The diagrams of $d B / d t-B$ and $\frac{\dot{B}}{B^{3}}-B$

unusual increases in spin-down torque as well as braking index $n$. Therefore, $\dot{P}$ also increases substantially, which can be illustrated by significant jumps in the period evolution of SGR 1900+14 after the 27 August 1998 giant flare (Marsden et al. 1999; Mereghetti et al. 2000). In short, for a SGR associated with a SNR, it is this change in configuration of the dipole magnetic field that could produce a significant deviation of $n(n>3)$ and cause the current value of $\dot{P}$ to be far larger than its mean value in history, the spin-down age will be far less than its real age. We can take SGR 1806-20 as an excellent example. From Table 3, the highest energy $E \sim(2-5) \times 10^{47}$ erg was released during the giant flare of December 27, 2004 from SGR 1806-20 which exceeded all previous giant/intermediate flares of SGRs. Among the known 23 magnetar, SGR 1806-20 has the strongest dipole magnetic field, $B=2.4 \times 10^{15} \mathrm{G}$, the largest period derivative, $\dot{P}=7.5 \times 10^{-10} \mathrm{~s} \mathrm{~s}^{-1}$, and the shortest spin-down or characteristic age, $t_{\text {Spin }}=0.16$ kyrs. All of this implies that the value of braking index of SGR 1806-20 is larger than the ideal value of $n=3$.

An alternative explain why all SGRs associated with SNRs appear younger than their real ages is provided in the follows. Glitches (sudden frequency jumps of a magnitude $\Delta \Omega / \Omega \sim 10^{9}$ to $10^{6}$, accompanied by the jumps of spin-down rates with a magnitude of
$\Delta \dot{\Omega} / \dot{\Omega} \sim 10^{-3}-10^{-2}$ )are common phenomenon in pulsars. After each glitch, a permanent increase in the pulsars spin-down rate usually happens, which implies that the pulsars surface magnetic field is also increased (be note, neither the period nor the spin-down rate is completely recovered although there is a relaxation after a glitch). Consequently, some radio pulsars with many active glitches may evolve into magnetars (Lin \& Zhang 2004; Chen \& Li 2006). As we know, magnetars show many similarities with typical radio pulsars, including the properties of glitches. The amplitudes of glitches of SGRs are far larger than those of AXPs and radio pulsars (Mereghetti 2008). The glitches of SGRs associated with SNRs could be triggered by stars' 'quaking', contributing to magnetars' giant/intermediate flares (Mereghetti 2008). The significant changes of spin-down rates and dipole magnetic field strengthes before and after giant flares of SGRs (0526-66, 1806-20, 1900+14 and 1627-41) associated with SNRs are good indications of huge glitches happened in four SGRs though some of these huge glitches are 'missed'(not reported) (Pons \& Rea 2012). Therefore, a SGR's present spin-down rate may be much higher than its initial value, and its characteristic age may be shorter than its true age. With respect to AXPs with SNRs, on one hand, their dipole magnetic fields 
Table 3 Giant/Intermediate flares from four SGRs.

\begin{tabular}{crrrr}
\hline Source & SGR $^{a} 0526-66$ & SGR $^{a} 1900+14$ & SGR $^{a}$ 1806-20 & SGR $^{b} 1627-41$ \\
\hline Date & March 5, 1979 & August 27, 1998 & December 27, 2004 & June 17, 1998 \\
Assumed Distance(kpc) & 55 & 15 & 15 & 5.8 \\
Peak Luminosity $(\mathrm{erg} / \mathrm{s})$ & $3.6 \times 10^{44}$ & $>1.5 \times 10^{44}$ & $(1.6 \sim 5) \times 10^{46}$ & $8 \times 10^{42}$ \\
Isotropic Energy $(\mathrm{erg})$ & $1.6 \times 10^{44}$ & $>8.3 \times 10^{44}$ & $(2 \sim 5) \times 10^{47}$ & $8 \times 10^{43}$ \\
\hline
\end{tabular}

Note: The sign 'a' denotes: The date are cited from (Mereghetti 2008). The sign 'b' denotes: The date are cited from (Mazets et al. 1999).

may also increase to a certain degree via glitches, on the other hand, the dipole magnetic fields rapidly decay via EC reaction, but the rates of increase are far less than the rates of decay. Thus, it's the dipolar magnetic field decay that plays an important role in making an AXP look older than it really is. It is worth noting that when a glitch happens, Eq.(23) no longer applies because the quantities of $\theta, I, R, \Omega($ or $P$ ) may vary to some extent, a part from $B$ increases.

In ZX2011, authors suggested that the characteristic age of a NS is not available to estimate its real age, and the physically meaningful criterion to estimate $t_{\text {Real }}$ is the NS-SNR association. Their suggestions are in the same way applicable to magnetars associated with SNRs, according to our analysis above.

\section{Conclusions}

In this paper, based on our modified model, we carry out a study of the magnetic field decay of magnetars in SNRs. The main conclusions are as follows:

1. In the presence of superhigh magnetic fields, the values of $\Gamma$ calculated by $\rho_{\mathrm{e}}$ derived in circular cylindrical coordinates are less than those of $\Gamma^{\prime}$ calculated by $\rho_{\mathrm{e}}^{\prime}$ deduced in spherical coordinates, due to the quantization of Landau levels. Combining the relation of $\Gamma=K(B) \Gamma^{\prime}$ with Landau level-superfluid modified factor $\Lambda$ yields a modified second-order differential equation for a superhigh magnetic field $B$ and its evolutionary timescale $t$.

2. Calculations show that the maximum of the field's decay timescale, $t \approx 2.9507 \times 10^{6}$ yrs when $B_{0}=$ $3.0 \times 10^{15} \mathrm{G}$ and $T_{0}=2.60 \times 10^{8} \mathrm{~K}$ (without considering the modified Urca reactions). Assuming different initial internal temperatures, the superhigh magnetic fields may evolve on timescales $\sim\left(10^{6} \sim 10^{7}\right)$ yrs for common magnetars.

3. On the basis of the results of the NS-SNR association of Zhang \& Xie (2011), we calculate the maximum initial internal magnetic fields of magnetar progenitors to be $\sim 2.0 \times 10^{14}-2.93 \times 10^{15} \mathrm{G}$, when $T_{0} \sim 2.60 \times 10^{8}$
K. If $T_{0} \sim 2.75 \times 10^{8}-1.75 \times 10^{8} \mathrm{~K}$, there still be $B_{\mathrm{i}} \sim\left(10^{14}-10^{15}\right) \mathrm{G}$, which are consistent with our model.

4. By means of statistical analysis, we found that an intense and significant positive correlation between $\log _{10}(\operatorname{SNR}($ Age $) /$ Spindown(Age) $)$ and $\log _{10} B$ for magnetars, and all AXPs associated with SNRs look older than their real ages, whereas all SGRs associated with SNRs appear younger than they are.

5 . We tentatively investigate the equation of braking index $n$ under pure magnetodipole radiation, and produce schematic diagrams of $\frac{d B}{d t}-B$ and $\frac{\dot{B}}{B^{3}}-B$ in a wide range of $B \sim 3.0 \times 10^{15} \sim 10^{12} \mathrm{G}$. According to our magnetar model, braking index $n$ could be correlated with both the dipole magnetic field and its decay rate.

6. 'The dipolar magnetic field decay plays a significant role in making a neutron star look older' suggested by Zhang and Xie (2011) is also applicable to magnetars. All AXPs may have experienced relatively 'normal' decay of their dipole magnetic fields, and thus have lower values of $n(n<3)$ and $\dot{P}$. In contrast, giant/intermediate flares were detected in SGRs associated with SNRs. Giant flares or huge glitches cause SGRs associated with SNRs spin down quickly, and make SGRs appear younger than their real ages.

Finally, due to the very little number of magnetars associated with SNRs, the above conclusions are tentative, and must be observationally validated.

Acknowledgements We thank the anonymous referee for the care in reading the manuscript and for useful comments which help us to improve this paper substantially, Prof. Xiang-Dong Li for his valuable suggestions on contents of this paper and helps on language, Profs. Yong-Feng Huang and Zi-Gao Dai for their helpful comments. This work is partly supported by Chinese National Science Foundation through grant No.10773005, National Basic Research Program of China (973 Program 2009CB824800), China Ministry of Science and Technology under State Key Development 
Program for Basic Research (2012CB821800), Knowledge Innovation Program of CAS $\mathrm{KJCX}_{2}-\mathrm{YW}$-T09, Xinjiang Natural Science Foundation No.2009211B35, the Key Directional Project of CAS and NSFC under projects 10173020, 10673021, 10773005, 10778631 and 10903019 . 


\section{References}

Aguilera, D. N., Pons, J. A., Miralles, J. A., 2008, Astrophys. J. Lett., 673, L167

Aharonian, F., Akhperjanian, A. G., Barres, de Almeida, U; et al., 2008, Astron. Astrophys.,486,829

Allen, M. P., Horvath, J. E., 1997, Astrophys. J., 488, 409

Allen, M. P., Horvath, J. E., 2004, Astrophys. J., 616, 346

Alpar, M. A., Ankay, A., Yazgan, E., 2001, Astrophys. J. Lett., 577, L61

Arras, P., Cumming, A., Thompson, C., 2004, Astrophys. J. Lett., 608, L49

Bardeen, J., Cooper, L. N., Schrieffer, J.R.,1957, Phys. Rev. 108, 1175

Blandford, R. D., Romani, R. W., 1988, Mon. Not. R. Astron. Soc., 234, 57

Braun, R., Goss, W. M., Lyne, A. G., 1989, Astrophys. J., 340,355

Camilo, F., Ransom, S. M., Halpern, J. P., Reynolds, J., 2007, Astrophys. J. Lett., 666, L93

Chen, W. C., Li, X. D., 2006, Astron. Astrophys., 450, L1

Corbel, S., Chapuis, C., Damc, T. M., Durouchoux, P.,1999, Astrophys. J. Lett., 526, L29

Duncan R. C., Thompson C., 1992, Astrophys. J., 392, L9

Duncan R. C., Thompson C., In: Rothschild R.E., Lingenfelter R.E. (eds.) High-Velocity Neutron Stars and Gamma-Ray Bursts. AIP Conference Proc., Vol. 366, p. 111. AIP Press, New York (1996)

Duncan R. C., 2000, arXiv:astro-ph/0002442

Elgarøy, Ø., et al., 1996, Phys. Rev. Lett., 77, 1482

Fahlman, G. G., Gregory, P. C., 1981, Nature, 293, 202

Gaensler, B. M., Gotthelf, E. V., Vasisht, G., 1999,Astrophys. J. Lett., 526, L37

Gaensler, B. M., Gotthelf, E. V., Vasisht, G., 2001,Astrophys. J., 559, 963

Gelfand J. D., Gaensler, B. M., 2007, Astrophys. J., 667, 1111 arXiv:0706.1054

Green, D. A., 1989, Mon. Not. R. Astron. Soc., 238, 737

Gao, Z. F., Wang, N., Yuan, J. P., et al., 2011a, Astrophys. Space Sci.,332, 129

Gao, Z. F., Wang, N., Yuan, J. P., et al., 2011b, Astrophys. Space Sci., 333, 427

Gao, Z. F., Wang, N., Song, D. L., et al., 2011c, Astrophys. Space Sci., 334, 281

Gao, Z. F., Peng, Q. H., Wang, N., et al., 2011d, Astrophys. Space Sci., 336, 427

Goldreich, P., Reisenegger, A., 1992, Astrophys. J., 395, 250

Gunn, J. E., Ostriker, J. P., 1970, Astrophys. J., 160, 979

Harding, A. K., Lai, D., 2006, Rep. on Pro. in Phys. 69, 2631

Halpern, J. P., Gotthelf, E. V., 2010, Astrophys. J., 725, 1384

Han, J. L., 1997, Astron. Astrophys., 489, 485

Heyl, J. S., Hernquist, L., 1997, Astrophys. J. Lett., 489, L67

Heyl, J. S., Kulkarni, S. R., 1998, Astrophys. J. Lett., 506, L61

Hurley, K., Kouveliotou, C., Woods, P., et al., 1999, Astrophys. J. Lett., 510, L107

Horiuchi, S., Suwa, Y., Takami, H., et al., 2008, Mon. Not. R. Astron. Soc., 391, 1893
Horvath, J. E., Allen, M. P., 2011, arXiv: 1104.2875v1

Ibrahim, A. I., Strohmayer, T. E., Woods, P. M., et al., 2001, Astrophys. J., 558, 237

Ibrahim, A. I., Markwardt, C. B., Swank, J. H, et al., 2004, Astrophys. J. Lett., 609, L1

Klose, S., Henden, A. A., Geppert, U., et al. 2004, Astrophys. J. Lett., 609, L13, arXiv:astro-ph/0405299

Kouveliotou, C., Fishman, G. J., Meegan, C. A., et al., 1994, Nature, 368, 125

Kulkarni, S. R., Frail, D. A.,1993,Nature, 365, 33

Kulkarni, S. R., Kaplan, D. L., Marshall, H. L., et al., 2003, Astrophys. J., 585, 948

Lenters, G. T., Woods, P. M., Goupell, J. E., et al., 2003, Astrophys. J., 587, 761

Lin, J. R., Zhang, S. N., 2004, Astrophys. J. Lett., 615, L133

Marsden, D., Rothschild, R. E., Lingenfelter, R. E., 1999, Astrophys. J. Lett., 520, 1107

Marsden, D., Lingenfelter, R. E., Rothschild, R. E., et al., 2001, Astrophys. J., 550, 397

Mazets, E. P., Aptekar, R. L., Butterworth, P. S., 1999, Astrophys. J. Lett., 519, L151

Mereghetti, S., Cremonesi, D., Feroci, M., Tavani, M., 2000, Astron. Astrophys., 361, 240

Menou, K., Perna, R., Hernquist, L., 2001, Astrophys. J. Lett., 554, L63

Mereghetti, S., Götz, D., Mirabel, I. F., et al., 2005, Astron. Astrophys., 433, L9

Mereghetti, S., 2008, Astron. Astrophys. Rev., 15, 225

Murakami, T., Tanaka, Y., Kulkarni, S. R., et al., 1994, Nature, 368, 127

Narayan, R., Ostriker, J. P., 1990, Astrophys. J., 352, 222

Ostriker, J. P., Gunn, J. E., 1969, Astrophys. J., 157, 1395

Peng, Q. H., Huang, K. L., Huang, J. H., 1982, Astron. Astrophys., 107, 258

Peng, Q. H., Tong H., 2007, Mon. Not. R. Astron. Soc., 378, 159

Peng Qiu He., Tong Hao., arXiv: 0911.2066v1 [astro-ph.HE] 11 Nov 2009, $10^{\text {th }}$ Symposium on Nuclei in the Cosmos, 27 July-1 August 2008 Mackinac Island, Michigan,USA

Pons, J. A., Link, B., Miralles, J. A., et al., 2006, arXiv: astro-ph/0607583v3

Pons, J. A., Miralles, J. A., Geppert, U., 2009, Astron. Astrophys., 496, 207

Pons, J. A., Rea, N., 2012,Astrophys. J. Lett., 750, 6

Rea, N., Esposito, P., Turolla, R., et al., 2010, Science, 330, 944

Rheinhardt, M., Geppert, U., 2003, Phys. Rev. Lett., 88, L101

Ridley, J. P., Lorimer, D. R., 2010, Mon. Not. R. Astron. Soc., 404, 1081

Rho, J., Petre, R., 1997, Astrophys. J.,484, 828

Sanbonmatsu, K. Y., Herfand, D. J., 1992,Astrophys. J., 104, 2189

Seward, F. d., 1990, Astrophys. J. Suppl. Ser., 73, 781

Shapiro, S. L., Teukolsky, S. A., 1983, 'Black holes, white drarfs, and neutron stars' John Wiley \& Sons, New York

Shull, J. M., Fesen, R. A., Saken, J. M., 1989, Astrophys. J., 346,860

Ternov, I., Lysov, B., Korovina, L., 1965, Moscow Univ. Phys. Bull. 5, 58

Thompson, C., Duncan, R. C., 1993, Astrophys. J., 543, 340 
Thompson, C., Duncan, R. C., 1996, Astrophys. J., 473, 322

Thompson, C., Duncan, R. C., Woods, P. M., 2000, Astrophys. J., 543, 340

Thompson, C., Lyutikov, M., Kulkami, S. R., 2002, Astrophys. J., 574, 332

van Paradijs, J., Taam, R. E., van den Heuvel, E. P. J., 1995, Astron. Astrophys., 299, 41

Vasisht, G., Kulkarni, S. R., Frail, D. A., et al., 2000, Astrophys. J. Lett., 431, L35

Vasisht, G., Gotthelf, E. V., 1997, Astrophys. J. Lett., 486, L129

Vasisht, G., Gotthelf, E. V., Torri, K., et al., 2000, Astrophys. J. Lett., 542, L49

Wacheter, S., Patel, S., Kouveliotou, C., et al., 2004, Astrophys. J., 615,887

White, N. E., Marshall, F. E., 1984,Astrophys. J., 281, 354

Wu, F., Xu, R. X., Gil, J., 2003, Astron. Astrophys., 409, 641

Xu, R. X., Qiao, G. J., 2001, Astrophys. J. Lett., 561, L85

Xu, R. X., Tao, D. J., Yang, Y., 2006, Mon. Not. R. Astron. Soc., 373, L85

Yakovlev, D. G., Kaminker A. D., Gnedin O. Y., et al., 2001, Phys. Rep., 354, 1

Zhang, B., Xu, R. X., Qiao, G. J., 2000, Astrophys. J. Lett., 545, L127

Zhang, S. N., Xie, Y., 2011, arXiv: 1110.3154v1 [astroph.HE] 


\section{Appendix}

\section{A An important assumption on the ${ }^{3} P_{2}$ neutron Cooper pairs}

The formation of Cooper pairs is a universal quantum-mechanical phenomenon of condensation in superfluids or superconductors. As pointed out in the original BCS work (Bardeen et al. 1957), pairing occurs basically between fermion states in the vicinity of the Fermi surface. Owing to pairing correlations, there is a major change in the low-energy spectrum of the system: A finite energy gap between its ground state and first excited state appears, and then the system will be subjected to a phase transition to a superfluid (or superconductor) state below a critical temperature.

From the analysis in Papers 1-4, the ${ }^{3} P_{2}$ neutron Cooper pairs can be destroyed by the outgoing EC neutrons easily. However, up to the present, the physics community has not yet produced the calculation of the collision probability at which an outgoing EC neutron destroys one ${ }^{3} P_{2}$ Cooper pair, due to special circumstances inside neutron stars, e.g., high temperatures, high-density matter, ultra-strong magnetic fields, and so on.

As a matter of fact, in the process of EC, for each degenerate species (electrons, protons, neutrons and neutrinos), only a fraction $\left(\sim k T / E_{\mathrm{F}}(i)\right)$ of particles lying in the vicinity of the Fermi surface can effectively contribute to the EC rate, $\Gamma$. Now, let us carry out the following evaluation: The number of neutrons participating in EC per unit volume, $n_{\mathrm{n}}^{\prime}$, is computed as

$n_{\mathrm{n}}^{\prime}=\frac{k T}{E_{\mathrm{F}}^{\prime}(n)} \int \rho_{\mathrm{n}} d E_{\mathrm{n}}=\frac{k T}{E_{\mathrm{F}}^{\prime}(n)} \times \frac{8 \pi \sqrt{2} m_{\mathrm{n}}^{3 / 2}}{h^{3}} \int_{E_{\mathrm{F}}^{\prime}(n)}^{\left\langle E_{\mathrm{n}}\right\rangle} E_{\mathrm{n}}^{\frac{1}{2}} d E_{\mathrm{n}}$.

As an illustration, we can arbitrarily assume $B=3.0 \times 10^{15} \mathrm{G}$ and $T=2.78 \times 10^{8} \mathrm{~K}$. From Paper 4, we obtain the following relations: $\left\langle E_{\mathrm{n}}\right\rangle=73.57 \mathrm{MeV}$, and $E_{\mathrm{F}}^{\prime}(n)=60 \mathrm{MeV}$ when $B=3.0 \times 10^{15} \mathrm{G}$. Eq.(A1) gives the value $n_{\mathrm{n}}^{\prime} \sim 2.377 \times 10^{34} \mathrm{~cm}^{-3}\left(\rho=\rho_{0}\right)$. Since the values we assumed are the possible maximum values of $B$ and $T$, the average value of $n_{\mathrm{n}}^{\prime}$ will be less than this value evaluated $\left(2.377 \times 10^{34} \mathrm{~cm}^{-3}\right)$, obviously. In the interior of a neutron star where the anisotropic ${ }^{3} P_{2}$ neutron superfluid exists, the neutron number density $n_{\mathrm{n}}=1.78 \times 10^{38} \mathrm{~cm}^{-3}$ when $\rho=\rho_{0}$ (Shapiro \& Teukolsky 1983), which indicates that both the number of neutrons and the number of the ${ }^{3} P_{2}$ neutron Cooper pairs per unit volume are far larger than the number of these newly formed (EC) neutrons per unit volume. Based on the above analysis, we may make a feasible assumption that each outgoing high-energy EC neutron can destroy one ${ }^{3} P_{2}$ neutron Cooper pair.

\section{B Necessary corrections and improvements in our previous work}

For the purpose of improving our magnetar model, we have checked our previous research in an all-round way. In this part, we make several necessary corrections in Paper 3, and provide key improvements in Paper 4.

In Paper 3, we derived the formulae for $E_{\mathrm{F}}(\mathrm{e})$ in superhigh magnetic fields, and concluded that the stronger the magnetic fields, the higher the electron Fermi energy becomes. However, the coefficient $\frac{2}{3}$ in Eq.(15) was wrongly treated as $\frac{3}{2}$ in the subsequent calculations, causing the formulae of $E_{\mathrm{F}}(r m e)$ deviate the actual case slightly. We honestly apologize to readers for our mistake. Now, the necessary corrections in Paper 3 are presented as follows: $\frac{3 \pi}{B^{*}}$ in Eqs.(16-19) must be replaced by $\frac{4 \pi}{3 B^{*}} ; \frac{(3 \pi)^{2}}{16 B^{*}}$ in Eq.(20) must be replaced by $\frac{\pi^{2}}{4 B^{*}} ;$ Eq.(23) can be rewritten as

$E_{\mathrm{F}}(\mathrm{e})=43.44\left[\frac{Y_{\mathrm{e}}}{0.0535} \frac{\rho}{\rho_{0}} \frac{B}{B_{\mathrm{cr}}}\right]^{\frac{1}{4}} \mathrm{MeV} \quad\left(B^{*} \geq 1\right)$.

To our pleasure, the higher value of $Y_{\mathrm{e}}=0.12$ (see Paper 4) will be replaced by the lower value $Y_{\mathrm{e}}=0.0535$. The later is slightly larger than the mean value of $Y_{\mathrm{e}}$ of a neutron star, $Y_{\mathrm{e}}=0.05$, and thus is plausible. Furthermore, the corrections of Eq.(20) and Eq.(24) in Paper 3 don't affect the calculated results of Paper 4. Now, some values of $E_{\mathrm{F}}(\mathrm{e})$ of Table 2 in Paper 3 are modified, shown as in Table 4. From the analysis in Paper 3, when $E_{F}(\mathrm{e}) \geq 5$ $\mathrm{MeV}$, the second term on the right of Eq.(20) can be ignored. This suggest that the second term on the right of Eq.(16) also can be ignored. Thus, the electron energy state density can be approximately expressed as

$N_{\mathrm{pha}} \approx \frac{4 \pi}{3 B^{*}}\left(\frac{m_{\mathrm{e}} c}{h}\right)^{3} \int_{0}^{\frac{E_{\mathrm{F}}(\mathrm{e})}{m_{\mathrm{e}} c^{2}}}\left[\left(\frac{E_{\mathrm{F}}(\mathrm{e})}{m_{\mathrm{e}} c^{2}}\right)^{2}-1-\left(\frac{p_{z}}{m_{\mathrm{e}} c}\right)^{2}\right]^{\frac{3}{2}} d\left(\frac{p_{z}}{m_{\mathrm{e}} c}\right)$. 
Table 4 The relation of $E_{F}(e)$ and $B$.

\begin{tabular}{lrrrrr}
\hline Name & $Y_{\mathrm{e}}$ & $E_{r m F}(1)$ & $E_{r m F}(2)$ & $E_{\mathrm{F}}(3)$ & $E_{\mathrm{F}}(4)$ \\
& & $B^{*}=0$ & $B^{*}=1$ & $B^{*}=10$ & $B^{*}=100$ \\
${ }_{26}^{56} \mathrm{Fe}$ & 0.4643 & 0.95 & $\mathbf{1 . 3 5}$ & $\mathbf{3 . 2 0}$ & $\mathbf{8 . 7 7}$ \\
\hline${ }_{28}^{62} \mathrm{Ni}$ & 0.4516 & 2.61 & $\mathbf{2 . 4 8}$ & $\mathbf{4 . 5 8}$ & $\mathbf{1 2 . 0 1}$ \\
${ }_{28}^{64} \mathrm{Ni}$ & 0.4375 & 4.31 & & $\mathbf{6 . 4 4}$ & $\mathbf{1 3 . 4 9}$ \\
${ }_{28}^{66} \mathrm{Ni}$ & 0.4242 & 4.45 & & $\mathbf{6 . 5 4}$ & $\mathrm{NO}$ \\
${ }_{386}^{86} \mathrm{Kr}$ & 0.4186 & 5.66 & & & $\mathbf{1 4 . 4 9}$ \\
${ }_{34}^{84} \mathrm{Se}$ & 0.4048 & 8.49 & & & $\mathbf{1 8 . 8 4}$ \\
${ }_{32}^{82} \mathrm{Ge}$ & 0.3902 & 11.44 & & & $\mathbf{2 1 . 7 5}$ \\
${ }_{30}^{80} \mathrm{Zn}$ & 0.3750 & 14.08 & & & $\mathbf{2 7 . 6 2}$ \\
\hline
\end{tabular}

Note: This table is cited from Table 2 of Paper 3. All the corrections are denoted in boldface.

Differentiating Eq.(B2) gives the following expression:

$\frac{d N_{\mathrm{pha}}}{d E_{\mathrm{e}}} d E_{\mathrm{e}} \simeq \frac{4 \pi}{3 B^{*}}\left(\frac{m_{\mathrm{e}} c}{h}\right)^{3} \frac{1}{m_{\mathrm{e}} c^{2}}\left[\left(\frac{E_{\mathrm{F}}(\mathrm{e})}{m_{\mathrm{e}} c^{2}}\right)^{2}-1-\left(\frac{E_{\mathrm{e}}}{m_{\mathrm{e}} c^{2}}\right)^{2}\right]^{\frac{3}{2}} d E_{\mathrm{e}}$.

Simplifying Eq.(B3) and using the relation $\rho_{\mathrm{e}}=\frac{d n_{\mathrm{e}}}{d E_{\mathrm{e}}}=\frac{d N_{\mathrm{pha}}}{d E_{\mathrm{e}}}$, we gain

$$
\begin{aligned}
& \rho_{\mathrm{e}}=\frac{4}{3} \frac{\pi}{B^{*}}\left(\frac{m_{\mathrm{e}} c}{h}\right)^{3} \frac{1}{m_{\mathrm{e}} c^{2}}\left[\left(\frac{E_{\mathrm{F}}(\mathrm{e})}{m_{\mathrm{e}} c^{2}}\right)^{2}-1-\left(\frac{E_{\mathrm{e}}}{m_{\mathrm{e}} c^{2}}\right)^{2}\right]^{\frac{3}{2}} \\
& =\frac{1}{3 B^{*}\left(2 \pi^{2} \hbar^{3} c^{3}\right)} \frac{1}{m_{\mathrm{e}} c^{2}}\left[E_{\mathrm{F}}^{2}(\mathrm{e})-0.261-E_{\mathrm{e}}^{2}\right]^{\frac{3}{2}},
\end{aligned}
$$

where $m_{\mathrm{e}} c^{2}=0.511 \mathrm{MeV}$ is used. Taking into account of gravitation redshift and utilizing Eq.(B1) and Eq.(B4), Eq.(16) and Eq.(19) in Paper 4 are modified as

$L_{\mathrm{X}}^{\infty}=\zeta(B, T)\left(1-r_{\mathrm{g}} / R\right) \frac{d E}{d t}$

and

$$
\begin{aligned}
& L_{\mathrm{X}}^{\infty} \simeq \Lambda(B, T) \zeta(B, T)\left(1-r_{\mathrm{g}} / R\right) \frac{4}{3} \pi R_{5}^{3} \frac{2 \pi}{\hbar} \frac{G_{\mathrm{F}}^{2} C_{\mathrm{V}}^{2}\left(1+3 a^{2}\right)}{2 \pi^{2} \hbar^{3} c^{3}} \frac{8 \pi \sqrt{2} m_{\mathrm{n}}^{\frac{3}{2}}}{h^{3}} \times \frac{\left(1.60 \times 10^{-6}\right)^{9.5}}{\left(2 \pi^{2} \hbar^{3} c^{3}\right) 3 B^{*} m_{\mathrm{e}} c^{2}} \\
& \times \int_{E_{\mathrm{F}}^{\prime}(\mathrm{n})}^{\left\langle E_{\mathrm{n}}\right\rangle} E_{\mathrm{n}}^{\frac{1}{2}}\left\langle E_{\mathrm{n}}\right\rangle d E_{\mathrm{n}} \int_{Q}^{E_{\mathrm{F}}(\mathrm{e})}\left[E_{\mathrm{F}}^{2}(\mathrm{e})-0.261-E_{\mathrm{e}}^{2}\right]^{\frac{3}{2}}\left(E_{\mathrm{e}}-Q\right)^{3} d E_{\mathrm{e}}
\end{aligned}
$$

respectively, where $L_{\mathrm{X}}^{\infty}$ is the apparent soft X-ray luminosity measured by a distant observer or the redshifted soft X-ray luminosity; $r_{\mathrm{g}}=2 G M / c^{2}=2.95 M / M_{\odot} \mathrm{km}$ is the Schwarzschild radius (we assume $R=10^{6} \mathrm{~cm}$ and $M=1.4 M_{\odot}$ for a canonical magnetar). We introduce the parameter $\phi(B, T)$ to denote $\Lambda(B, T) \zeta(B, T)$ in Eq.(B6), the value of $\phi(B, T)$ of a magnetar can be evaluated by combining Eq.(B6) with Table 4 of Paper 4 . Fig.9 shows the distribution of $\phi(B, T)$ of 7 canonic magnetars, whose persistent soft X-ray luminosities should not be less than their rotational energy loss rates $d E / d t$ (Paper 4$)$. It is worth noting that the fitting curve of $\phi(B, T)$ as a function of $B$ and $T$ cannot be obtained from Fig.9 because of the very little number of canonic magnetars. For each canonical magnetar with a given soft X-ray luminosity, its value of $\phi$ is mainly determined by $B$, and is insensitive to $T$, though $\phi$ is a function of $B$ and $T$. The mean value of $\phi(B, T)$ of 7 magnetars is calculated to be 


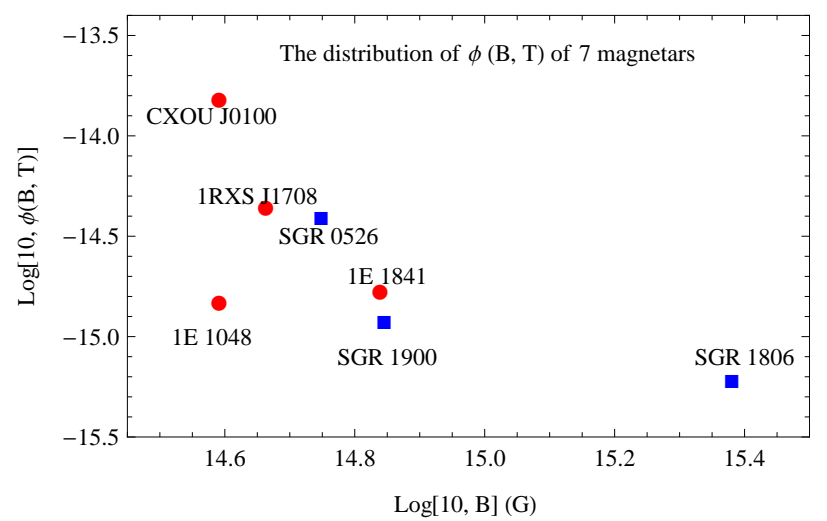

Fig. 9 The distribution of $\phi(B, T)$ of 7 canonic magnetars when $B_{0}=3.0 \times 10^{15} \mathrm{G}$ and $T_{0}=2.6 \times 10^{8} \mathrm{~K}$. The range of $B$ is assumed to be $\left(1.80 \times 10^{14} \sim 3.0 \times 10^{15} \mathrm{G}\right.$, arbitrarily. Circles and squares are for AXPs and SGRS, respectively.

$2.54 \times 10^{-15}$ by using the expression of $\langle\phi\rangle=\frac{\sum B_{i} \phi_{i}}{\sum B_{i}}$. Combining Eq.(7) with $\langle\phi\rangle$ gives

$$
\begin{aligned}
& \phi(B, T) \approx \frac{2.54 \times 10^{-15}}{3.198 \times 10^{-14}} \times\left(2.60388 \times 10^{-14}+1.98233 \times 10^{-30} B\right) \\
& \left(\frac{T_{0}}{2.6 \times 10^{8} \mathrm{~K}}\right)^{4} \operatorname{Exp}\left[\frac{-0.048 \mathrm{MeV}}{k}\left(\frac{1}{T_{0}}-\frac{1}{2.6 \times 10^{8} \mathrm{~K}}\right)\right] \\
& =\left(2.068 \times 10^{-15}+1.5747 \times 10^{-31} B\right)\left(\frac{T_{0}}{2.6 \times 10^{8} \mathrm{~K}}\right)^{4} \\
& \operatorname{Exp}\left[\frac{-0.048 \mathrm{MeV}}{k}\left(\frac{1}{T_{0}}-\frac{1}{2.6 \times 10^{8} \mathrm{~K}}\right)\right] .
\end{aligned}
$$

Inserting Eq.(B7) into Eq.(B6), we calculate the values of $L_{\mathrm{X}}^{\infty}$ in superhigh magnetic fields, $L_{\mathrm{X}}^{\infty} \sim\left(5.457 \times 10^{28}-\right.$ $\left.3.834 \times 10^{37}\right) \mathrm{erg} \mathrm{s}^{-1}$, corresponding to $B \sim\left(1.8 \times 10^{14}-3.0 \times 10^{15}\right)$ G. Furthermore, the calculated results of $L_{\mathrm{X}}^{\infty}$ are compared with those of $L_{\mathrm{X}}$ of Paper 4, shown as in Fig.10 From Fig.10, the values of $L_{\mathrm{X}}^{\infty}$ are slightly less than

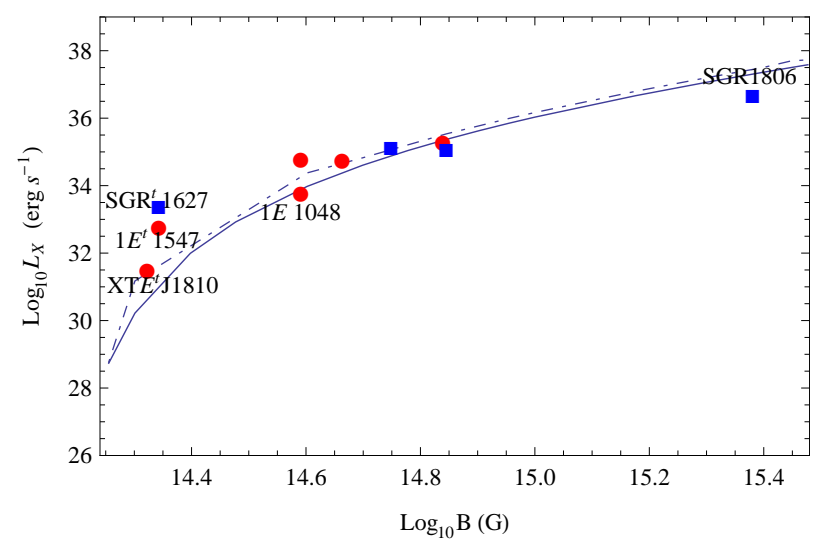

Fig. 10 The diagrams of soft X-ray luminosity as a function of magnetic field strength $B$ when $B_{0}=3.0 \times 10^{15} \mathrm{G}$ and $T_{0}=2.6 \times 10^{8} \mathrm{~K}$. The range of $B$ is assumed to be $\left(1.80 \times 10^{14} \sim 3.0 \times 10^{15} \mathrm{G}\right.$, arbitrarily. Circles and squares are for AXPs and SGRS, respectively. Solid line and dot-dashed line are for the modified luminosities and unmodified luminosities (Paper 4), respectively.

those of $L_{\mathrm{X}}$, the main reason for this is that the factor of gravitation redshift is considered in calculating $L_{\mathrm{X}}^{\infty}$. 


\title{
Magnetic field decay of magnetars in supernova remnants
}

\author{
Z. F. Gao ${ }^{1,2,3}$ • Q. H. Peng ${ }^{4} \cdot$ N. Wang ${ }^{1,2} \bullet$ \\ J. P. Yuan ${ }^{1,2}$
}

\begin{abstract}
In this paper, we modify our previous research carefully, and derive a new expression of electron energy density in superhigh magnetic fields. Based on our improved model, we re-compute the electron capture rates and the magnetic fields' evolutionary timescales $t$ of magnetars. According to the calculated results, the superhigh magnetic fields may evolve on timescales $\sim\left(10^{6}-10^{7}\right)$ yrs for common magnetars, and the maximum timescale of the field decay, $t \approx 2.9507 \times 10^{6} \mathrm{yrs}$, corresponding to an initial internal magnetic field $B_{0}=3.0 \times 10^{15} \mathrm{G}$ and an initial inner temperature $T_{0}=2.6 \times 10^{8} \mathrm{~K}$. Motivated by the results of the neutron star-supernova remnant(SNR) association of Zhang \& Xie(2011), we calculate the maximum $B_{0}$ of magnetar progenitors, $B_{\max } \sim\left(2.0 \times 10^{14}-2.93 \times 10^{15}\right) \mathrm{G}$ when $T_{0}=2.6 \times 10^{8}$ $\mathrm{K}$. When $T_{0} \sim 2.75 \times 10^{8}-1.75 \times 10^{8} \mathrm{~K}$, the maximum $B_{0}$ will also be in the range of $\sim 10^{14}-10^{15} \mathrm{G}$, not
\end{abstract}

\footnotetext{
Z. F. Gao

${ }^{1}$ Xinjiang Astronomical Observatory, CAS, 150, Science 1-Street, Urumqi Xinjiang, 830011,China zhifu_gao@uao.ac.cn

${ }^{2}$ Key Laboratory of Radio Astronomy, Chinese Academy of SciencesNanjing, 210008, China

${ }^{3}$ Graduate University of the Chinese Academy of Sciences, 19A Yuquan Road, Beijing, 100049, China

Q. H. Peng

${ }^{4}$ Department of Astronomy, Nanjing University, Nanjing, 210093. China

N. Wang

${ }^{1}$ Xinjiang Astronomical Observatory, CAS, 150, Science 1-Street, Urumqi Xinjiang, 830011, China

${ }^{2}$ Key Laboratory of Radio Astronomy, Chinese Academy of SciencesNanjing, 210008, China

J. P. Yuan

${ }^{1}$ Xinjiang Astronomical Observatory, CAS, 150, Science 1-Street, Urumqi Xinjiang, 830011, China

${ }^{2}$ Key Laboratory of Radio Astronomy, Chinese Academy of SciencesNanjing, 210008, China
}

exceeding the upper limit of magnetic field of a magnetar under our magnetar model. We also investigate the relationship between the spin-down ages of magnetars and the ages of their SNRs, and explain why all AXPs associated with SNRs look older than their real ages, whereas all SGRs associated with SNRs appear younger than they are.

Keywords Magnetar. Electron capture rate. Supernova remnant. and Superhigh magnetic field

\section{Introduction}

Recent developments have shown that a substantial fraction of newly born stars have magnetic field strengths in excess of the quantum critical value, $B_{\text {cr }}=4.414 \times 10^{13} \mathrm{G}$, above which the effect of Landau quantization on the transverse electron motion becomes considerable(Ternov et al. 1965). We divide them into two glasses- Soft Gamma-ray Repeaters(SGRs) and Anomalous X-ray Pulsars (AXPs) through the studies of their emission mechanisms. The SGRs and AXPs are currently considered to be 'magnetars', powered by extremely strong magnetic fields, rather than by their spin-down energy loss, as is the case for common radio pulsars (Duncan \& Thompson 1992; Thompson \& Duncan 1993; Thompson \& Duncan 1996).

A supernova remnant(SNR) is an expanding diffuse gaseous nebula that results from the explosion of a massive star. To date, there are 23 detected magnetar candidates: 11 SGRs (7 confirmed), and 12 AXPs (9 confirmed). Of the magnetar candidates, 4 SGRs and 5 AXPs (more than a third) are associated with the known SNRs, suggestive of an origin in massive star explosions (Gaensler et al. 2001; Marsden et al. 2001; Allen \& Horvath 2004; Mereghetti 2008). Estimations indicate that about $10 \%$ of supernova explosions may 
lead to a magnetar (Kouveliotou et al. 1994). If magnetars are from core-collapse supernovae, as estimated above, then their magnetic fields could have been inherited from their progenitors (Horiuchi et al. 2008).

The SGRs, characterized primarily by their occasional repeating bursts of soft $\gamma$-rays, have spin periods $(5 \sim 8)$ s, positive period derivatives, and persistent soft X-ray luminosities $\sim 10^{35} \mathrm{erg} \mathrm{s}^{-1}$ (Duncan 2000; Marsden et al. 2001). There are four known SGRs associated with SNRs: O526-66, 1900+14, 180620 and 1627-41. SGR 0526-66 has been associated with N49 in the Large Magellanic Cloud Kulkarni et al. 2003). SGR 1806-20 and SGR 1627-41 apparently lie in G10.0-0.3 (Kulkarni \& Frail 1993) and G33.70-01 (Corbel et al. 1999), respectively. SGR $1900+14$ is associated with G42.8+0.6 (Hurley et al. 1999). However, another SNR G43.9+1.6 also falls within the error box of SGR 1900+14 (Vasisht et al. 1994), so a great deal of effort will be required to investigate whether this magnetar is stably associated with G42.8+0.6.

The AXPs, so called due to their high X-ray luminosities, $10^{34} \sim 10^{36}$ erg $\mathrm{s}^{-1}$, the lack of evidence of binary companions van Paradijs et al. 1995; Duncan \& Thompson 1996). To date, there are five AXPs are located near the centers of SNRs: 1E 2259+586 in CTB 109 (Fahlman \& Gregorv 1981), 1E 1841-045 in Kes 73 (Sanbonmatsu \& Herfand 1992), 1E 1547.0-5408 in G327.24-0.13 (Camilo et al. 2007); CXOU J171405.7-381031 in CTB37B (Aharonian et al. 2008) and AX J1845-0258 in G29.6+0.1 (Gaensler et al. 1999).

Due to a very little number of magnetars (only 16 confirmed currently), some puzzles concerning supernova progenitors and their birth events that confront us seem inevitable. However, a few confirmable magnetar/SNR associations still remain undisputed Gaensler et al. 2001; Marsden et al. 2001; Allen \& Horvath 2004). These associations are confirming that a magnetar candidate was formed in a supernova explosion, and is thus thought to be the collapsed core of a massive star, which is likely to be a neutron star(NS). Since a NS and its associated SNR are from the same explosion, they should have the same age (Zhang \& Xie 2011). All the known SNRs of both AXPs and SGRs are comparatively young, $t_{\mathrm{SNR}} \sim$ several kyr (see Sec.3), which infers that magnetars are also very young objects (Shull et al. 1989; Vasisht \& Gotthelf 1997).

Over the last decades, radio pulsars(here, observed as normal radio pulsars) with the possible magnetic field evolutionary timescale, $t \sim 10^{6}-10^{7} \mathrm{yrs}$, have been studied extensively using the statistical distribution in the $P-\dot{P}$ diagram (Ostriker \& Gunn 1969;

Gunn \& Ostriker|1970; Shull et al. 1989; [Narayan \& Ostriker] magnetars - ' $\alpha-\Omega$ dynamo' (Duncan \& Thompson
1990; Han 1997; Ibrahim et al. 2004; Aguilera et al. 2008; Ridley \& Lorimer 2010; Zhang \& Xie 2011). Maybe radio pulsars were born with different initial external circumstances and initial internal conditions, these pulsars have experienced different evolutionary routes: the magnetic fields remain nearly constant in about half of them, decrease rapidly in others, or increase in very few pulsars (Han 1997; Zhang \& Xie 2011).

In order to explain the evolution of superhigh magnetic fields inside magnetars, different models have been proposed recently, as partly listed below. According to the twisted magnetospheres model, the unwinding of the internal field shears the star's crust, the rotational crustal motions generally provide a source of helicity for the external magnetosphere by twisting the magnetic fields which are anchored to the star's surface, drive currents outside a magnetar, and generate X-ray emissions (Thompson et al. 2000). The sudden crustal fractures (or starquakes) caused by unbearable stress can provide plausible mechanisms for magnetar outbursts and giant flares (Thompson et al. 2000, 2002). In addition, the overall evolution of SGR $1806-20$ in the years preceding the giant flare of December 27, 2004 seems to support some predictions of this model (Mereghetti et al. 2005).

In the thermal evolution model, the field could decay directly as a result of the non-zero resistivity of the matter through Ohmic decay or ambipolar diffusion, or indirectly as a result of Hall drift producing a cascade of the field to high wave number components, which decay quickly through Ohmic decay Goldreich \& Reisenegger 1992; Rheinhardt \& Geppert 2003; ?); magnetic field decay can be a main source of internal heating (Pons et al. 2009; Arras et al. 2004); the enhanced thermal conductivity in the strongly magnetized envelope contributes to raise the surface h mperature (Heyl \& Hernquist 1997; Heyl \& Kulkarni 1998). Based on this model, the surface thermal temperature of a magnetar is estimated to be $\sim\left(10^{5}-10^{6}\right)$ $\mathrm{K}$, which is basically consistent with the observations (Heyl \& Kulkarni 1998; Pons et al. 2009).

Although there are apparent advantages in some magnetar models, including the above two magnetar models, substantial improvements must be made for these models. The main disadvantages of these models can be summarized as follows: (1) not considering the effects of anisotropic ${ }^{3} P_{2}$ neutron superfluid (mainly in the outer core) on the decay of superhigh magnetic fields; (2) not combining the ages of SNRs with the timescales of magnetic fields' evolution; (3) universally adopting the most popular assumption on the origin of superhigh magnetic fields 
1992; Thompson \& Duncan 1993), which is a mere assumption laking observational support (Gao et al. 2011a, b, c, d) (hereinafter Paper 1, Paper 2, Paper 3 and Paper 4, respectively).

Unlike other magnetar models, we propose that superhigh magnetic fields of magnetars originate from the induced magnetic fields below a critical temperature, and the maximum field strength is $\sim(3.0-4.0) \times 10^{15}$ G (Peng \& Tong 2007; Peng \& Tong 2009). In the initial stage of our magnetar model, the major conclusions are briefly summarized as following: In Paper 1 , we numerically simulated the whole process of electron capture (EC); in order to calculate the effective electron capture rates, $\Gamma_{\text {eff }}$, we introduced the Landau level effect coefficient, $q$, whose magnitude is evaluated to be $\sim 10^{-18}$ by comparing the observed magnetars' soft X-ray luminosities $L_{\mathrm{X}}$ with the calculated values of $L_{\mathrm{X}}$. In Paper 2, superhigh magnetic fields give rise to an increase in the electron Fermi energy $E_{\mathrm{F}}(\mathrm{e})$, which will induce EC inside a magnetar. The ${ }^{3} P_{2}$ Cooper pairs with the maximum binding energy of $0.048 \mathrm{MeV}$ (Elgarøy et al. 1996) will be destroyed by the outgoing high-energy EC neutrons. Then the magnetic moments of the ${ }^{3} P_{2}$ Cooper pairs destroyed are no longer arranged in the paramagnetic direction, so the superhigh magnetic fields produced by the aligned magnetic moments of the ${ }^{3} P_{2}$ Cooper pairs will disappear gradually. Combining parameter $q$ with ${ }^{3} P_{2}$ anisotropic neutron superfluid theory yields a second-order differential equation for superhigh magnetic fields $B$ and their evolution timescales $t$. In Paper 3, by introducing the Dirac $\delta$-function, we deduced a general formula for $E_{\mathrm{F}}(\mathrm{e})$, which is suitable for extremely intense magnetic fields. In Paper 4, we presented the mechanism for the magnetar soft $\mathrm{X} / \gamma$-ray emission, numerically simulated the process of magnetar cooling and magnetic field decay, and then computed $L_{\mathrm{X}}$ of magnetars by introducing two important parameters: Landau level-superfluid modified factor $\Lambda$ and effective $\mathrm{X} / \gamma$-ray coefficient $\zeta$.

In this work, we re-examine our previous research carefully, and find that the expression of energy state density $\rho_{\mathrm{e}}$ of electrons in superhigh magnetic fields, as that of $E_{\mathrm{F}}(\mathrm{e})$, should be derived in circular cylindrical coordinates rather than in spherical coordinates, because the Landau column becomes a very long and narrow cylinder along the magnetic field. In Appendix $\mathrm{B}$ of this article, we modify the expression of $E_{\mathrm{F}}(\mathrm{e})$, derive a new formula of electron energy state density in superhigh magnetic fields, improve the calculated results of $L_{\mathrm{X}}$ and $\zeta$, and compare these results with those of Paper 4. Apart from the modifications above, the expression of $\Gamma$, together with the second-order differential equation for $B$ and $t$ in Paper 2, must be improved.
The main reasons are as follows:

1. In the interior of a magnetar, the process of EC is a relatively independent process, and is irrelevant to the luminosity $L_{\mathrm{X}}$, the values of $\Gamma$ are completely determined by inner physical properties, e.g., magnetic field strength, matter density, temperature, neutron superfluid and so on.

2. Since the magnitude of $q$ can be estimated by the observed luminosities $L_{\mathrm{X}}$ (see Paper 1), in fact, $q$ have included the influences of all the following factors: neutron superfluid's restraining effect, the fractions of all particles participating in EC reaction, thermal energy loss, energy conversion efficiency, and gravitation redshift, accidentally. However, if the influences of the above factors are considered, the real value of $q$ will be far less than that of Paper 1, therefore, $q$ is no longer used by our improved model. In Paper 4, $q$ has been replaced by two important parameters: Landau level-superfluid modified factor, $\Lambda$, and effective $\mathrm{X} / \gamma$-ray coefficient, $\zeta$ when calculating $L_{\mathrm{X}}$.

3. In our previous work (Papers 1-4), all the expressions of $\Gamma$, however, do not make use of the quantity $\Lambda$ and the new expression of $\rho_{\mathrm{e}}$ in superhigh magnetic fields (see Appendix B), so these expressions of $\Gamma$ are not consistent with the actual circumstances inside magnetars. All of these strongly suggest a necessity of reconsidering $\Gamma$ and the equation of $B$ and $t$.

In 2011, Shuang-Nan Zhang and Yi Xie published an article titled 'Magnetic field decay makes NSs look older than they are', which showed convincing evidence of magnetic field decay in some young NSs, and reasoned that the magnetic field decay can change substantially their spinning behaviors, and as a result these NSs appear much older than their real ages (Zhang \& Xie 2011) (hereinafter referred to as ZX2011). According to ZX2011, a NS and its associated SNR should have the same age, i.e., $t_{\text {Real }}=t_{\text {SNR; }}$ the NS's spin-down or characteristic age can be generally expressed as, $t_{\text {Spin }}=P /(n-1) \dot{P}$, where $P, \dot{P}$ and $n$ are its spin period, period derivative and braking index, respectively; however, $n$ always deviates from the the value $(n=3)$ expected for pure magnetic dipole radiation model. The authors supposed that, $n \gg 3$ is required for neutrons with $t_{\text {Spin }} \ll t_{\text {SNR }}$ if there is no significant magnetic field decay (note: this case is not believed to be plausible by authors), $n<3$ makes the spin-down age of a NS even longer than assuming $n=3$, and in this case more or even all all NSs have $t_{\text {Spin }} \ll t_{\text {SNR }}$; for any reasonable values of $n$, at least some of these NSs must have experienced significant dipole magnetic 
field decay. Furthermore, magnetic field decay dominated by the ambipolar diffusion has been investigated, and the core and surface temperatures of a NS have been estimated, whose results are agreed qualitatively with observations (Pons et al.2009). However, authors did not provide the observation data of magnetars, and thus omitted to explain why all AXPs associated with SNRs look older than their real ages, whereas all SGRs associated with SNRs appear younger than they are.

The remainder of this paper is organized as follows. In Sec.2, by introducing two different types of electron energy state density, the electron capture rates $\Gamma$ in superhigh magnetic fields are calculated, and the calculated results are compared with each other. In Sec.3.1, the differential equation of $B$ and $t$ is modified, and the values of $t$ are re-computed. In Sec.3.2, the maximum initial internal fields of magnetar progenitors are computed taking $t_{\text {Real }}=t_{\text {SNR }}$ proposed by ZX2011 as the starting point. In Sec.3.3, the relationship between the spin-down ages of magnetars and the ages of their SNRs are investigated. In Sec.4, a brief summary is given. In Appendix A, an important assumption on the ${ }^{3} P_{2}$ neutron Cooper pairs is presented, and several corrections and improvements in our models are presented in Appendix B.

\section{Electron capture rates in superhigh magnetic fields}

Since the quantized microstates don't exist in the momentum (or energy) space between the $n$-th and $(n+1)$ th Landau level, the Dirac $\delta$-function must be taken into account when calculating $E_{\mathrm{F}}(\mathrm{e})$ in superhigh magnetic fields. From Appendix B, a concise expression for $E_{\mathrm{F}}(\mathrm{e})$ in superhigh magnetic fields is of the form,

$E_{\mathrm{F}}(\mathrm{e}) \simeq 43.44\left(\frac{B}{B_{\mathrm{cr}}}\right)^{\frac{1}{4}}\left(\frac{\rho}{\rho_{0}} \frac{Y_{\mathrm{e}}}{0.0535}\right)^{\frac{1}{4}} \quad \mathrm{MeV}$.

where $\rho_{0}=2.8 \times 10^{14} \mathrm{~g} \mathrm{~cm}^{3}$ is the standard nuclear density. In order to calculate the $\mathrm{EC}$ rate, $\Gamma$ in a magnetar, we concentrate on non-relativistic, degenerate nuclear matter and super-relativistic, degenerate electrons. In the case of $0.5 \rho_{0} \leq \rho \leq 2 \rho_{0}$, the following expressions hold approximately: $E_{\mathrm{F}}^{\prime}(\mathrm{n})=$ $60\left(\rho / \rho_{0}\right)^{\frac{2}{3}} \mathrm{MeV}$ and $E_{\mathrm{F}}^{\prime}(\mathrm{p})=1.9\left(\rho / \rho_{0}\right)^{\frac{4}{3}} \mathrm{MeV}$, where $E_{\mathrm{F}}^{\prime}(\mathrm{n})$ and $E_{\mathrm{F}}^{\prime}(\mathrm{p})$ are the neutron Fermi kinetic energy and the proton Fermi kinetic energy, respectively (Shapiro \& Teukolsky 1983). In this paper, for convenience, we set $\rho=\rho_{0}$ and the electron fraction $Y_{\mathrm{e}}=0.0535$ in all the following calculations. This choice yields the threshold energy of EC reaction, $Q=E_{\mathrm{F}}(\mathrm{n})-E_{\mathrm{F}}(\mathrm{p})=59.39 \mathrm{MeV}$. The range of $B$ is assumed to be $B \sim\left(B_{\text {th }} \sim 3.0 \times 10^{15} \mathrm{G}\right)$, where $B_{\mathrm{th}}=1.5423 \times 10^{14} \mathrm{G}$ is the threshold magnetic field of $\mathrm{EC}$ reaction, corresponding to $E_{F}(\mathrm{e})=59.39 \mathrm{MeV}$. Thus, the range of $E_{\mathrm{e}}$ is $\left(Q \sim E_{\mathrm{F}}(\mathrm{e})\right)$. By employing energy conservation via $E_{\nu}+E_{\mathrm{n}}=E_{\mathrm{e}}+E_{\mathrm{p}}$, the Fermi energy of neutrinos, $E_{\mathrm{F}}(\nu)$, can be calculated by

$$
E_{\mathrm{F}}(\nu)=E_{\mathrm{F}}(\mathrm{e})-Q=E_{\mathrm{F}}(\mathrm{e})-59.39 \quad \mathrm{MeV} .
$$

According to our point of view, once the energies of electrons near the Fermi surface exceed $Q$, the EC reaction will dominate (see Paper 2 and Paper 4). From Appendix B, the energy state density of electrons in superhigh magnetic fields is of the form:

$$
\begin{aligned}
& \rho_{\mathrm{e}} \simeq \frac{4}{3} \frac{\pi}{B^{*}}\left(\frac{m_{\mathrm{e}} c}{h}\right)^{3} \frac{1}{m_{\mathrm{e}} c^{2}}\left[\left(\frac{E_{F}(\mathrm{e})}{m_{\mathrm{e}} c^{2}}\right)^{2}-1-\left(\frac{E_{\mathrm{e}}}{m_{\mathrm{e}} c^{2}}\right)^{2}\right]^{\frac{3}{2}} \\
& =\frac{1}{3 B^{*}\left(2 \pi^{2} \hbar^{3} c^{3}\right)} \frac{1}{m_{\mathrm{e}} c^{2}}\left[E_{F}^{2}(\mathrm{e})-0.261-E_{\mathrm{e}}^{2}\right]^{\frac{3}{2}} .(3)
\end{aligned}
$$

where $B^{*}$ is a non-dimensional magnetic field, defined as $B^{*}=B / B_{\mathrm{cr}}$. Since neutrinos/antineutrinos are uncharged, the energy state density of neutrinos (antineutrinos) remains unchanged,

$\rho_{\nu}=\frac{\left(E_{\mathrm{e}}-Q\right)^{2}}{2 \pi^{2} \hbar^{3} c^{3}}$.

The EC rate $\Gamma$, defined as the number of electrons captured by one proton per second, can be computed using the standard charged-current $\beta$-decay theory (Shapiro \& Teukolsky 1983). However, for each degenerate species, only a fraction $\left(\sim k T / E_{\mathrm{F}}(\mathrm{i})\right)$ of particles near the Fermi surface can effectively contribute to $\Gamma$. In Paper 4, we introduced the 'Landau levelsuperfluid modified factor' $\Lambda$,

$\Lambda=\frac{(k T)^{4} \operatorname{Exp}\left(-\Delta_{\max }\left({ }^{3} P_{2}\right) / k T\right)}{E_{\mathrm{F}}^{\prime}(\mathrm{n}) E_{\mathrm{F}}^{\prime}(\mathrm{p}) E_{\mathrm{F}}(\mathrm{e}) E_{\mathrm{F}}\left(\nu_{\mathrm{e}}\right)}$.

The initial conditions (e.g., $B, T$ ) of the magnetars are likely to be very different, implying that their values of $\Lambda$ are also different. For convenience, we assume a uniform initial magnetic field $B_{0}=3.0 \times 10^{15} \mathrm{G}$ for magnetar progenitors. Since the process of EC is a precess of magnetic field decay and inner cooling, when electrons are captured, the numbers of particles participating in EC near the Fermi surfaces decrease, which leads to a decrease in $\Lambda$. However, in the interior of a magnetar, the $\beta$ - decay and the inverse $\beta$-decay occur simultaneously as required by the charge neutrality, so when B decays, the depleted protons and electrons are recruited many times, which leads to only a small decrease in $Y_{\mathrm{e}}$ and $Y_{\mathrm{p}}$. In addition, the electrons are super-relativistic and degenerate, when the internal 
temperature falls, the electron transition between Landau levels is not permitted, because the electrons can be approximately treated as a zero-temperature Fermi gas. Thus, the value of $\Lambda$ decreases very slowly. In order to obtain a fitting function of $\Lambda, B$ and $T$, we numerically simulate the inner cooling and the magnetic field decay. Since the internal temperature of a magnetar is $\sim 10^{8} \mathrm{~K}$ (Yakovlev et al. 2001), and the maximum initial temperature (not including the inner core temperature) cannot exceed the critical temperature of the ${ }^{3} P_{2}$ Cooper pairs $T_{\text {cn }} \sim \Delta_{\max }\left({ }^{3} P_{2}\right) / k T \sim 2.78 \times 10^{8} \mathrm{~K}(\mathrm{~Pa}-$ per 4 ), we can arbitrarily assume $T_{0}$ to be $2.60 \times 10^{8} \mathrm{~K}$, corresponding to an initial value of $\Lambda \sim 3.198 \times 10^{-14}$. Then, we gain

$$
\begin{aligned}
& \Lambda\left(B_{0}, T_{0}\right)=\Lambda\left(T_{0}\right)=3.198 \times 10^{-14}\left(\frac{T_{0}}{2.6 \times 10^{8} \mathrm{~K}}\right)^{4} \\
& \operatorname{Exp}\left[\frac{-0.048 \mathrm{MeV}}{k}\left(\frac{1}{T_{0}}-\frac{1}{2.6 \times 10^{8} \mathrm{~K}}\right)\right] .
\end{aligned}
$$

When $B$ decreases from $B_{0}$ to $B_{\text {th }}$, the ratio of $\left|\frac{\Delta T}{\Delta B}\right|_{\max } \sim\left|\frac{\left(2.78 \times 10^{8}-1.0 \times 10^{8}\right) \mathrm{K}}{\left(3.0 \times 10^{15}-1.5423 \times 10^{14}\right) \mathrm{G}}\right| \sim 5.33 \times 10^{-8} \mathrm{~K} / \mathrm{G}$. When simulating numerically, the assumed value of $\left|\frac{\Delta T}{\Delta B}\right|$ cannot be too high or too small, otherwise the internal temperature $T$ drops wildly (eg., $T \ll 10^{7} \mathrm{~K}$ ) or insignificantly. According to the analysis above, we arbitrarily set $B_{0}=3.0 \times 10^{15}, T_{0}=2.60 \times 10^{8} \mathrm{~K}$ and $\Lambda\left(B_{0}, T_{0}\right)=3.198 \times 10^{-14}$ (these specific values are representative of the initial conditions encountered), and $T$ is decreased by step $\Delta T=3.5 \times 10^{4} \mathrm{~K}$. The results of numerical simulations are shown in Fig.1. From Fig. 1 a and Fig.1 b, both $\Lambda$ and $T$ decrease with decreasing $B$. When $\left.B \sim 3.0 \times 10^{15}-2.0 \times 10^{14}\right) \mathrm{G}$, $\Lambda \sim 3.198 \times 10^{-14}-2.649 \times 10^{-14}$. Base on the simulations above we gain

$$
\begin{aligned}
& \Lambda(B, T) \approx\left(2.60388 \times 10^{-14}+1.98233\right. \\
& \left.\times 10^{-30} B\right)\left(\frac{T_{0}}{2.6 \times 10^{8} \mathrm{~K}}\right)^{4} \operatorname{Exp}\left[\frac{-0.048 \mathrm{MeV}}{k}\right. \\
& \left.\left(\frac{1}{T_{0}}-\frac{1}{2.6 \times 10^{8} \mathrm{~K}}\right)\right],
\end{aligned}
$$

where $B_{0}=3.0 \times 10^{-15} \mathrm{G}$ is used. In the same way, we obtain a diagram of $\Lambda$ as a function of internal temperature $T$, as shown in Fig.2. From Fig.2, when internal temperature $T$ drops from $2.60 \times 10^{8} \mathrm{~K}$ to $1.52 \times 10^{8}$ $\mathrm{K}, \Lambda$ decreases, but its order of magnitude remains unchanged.

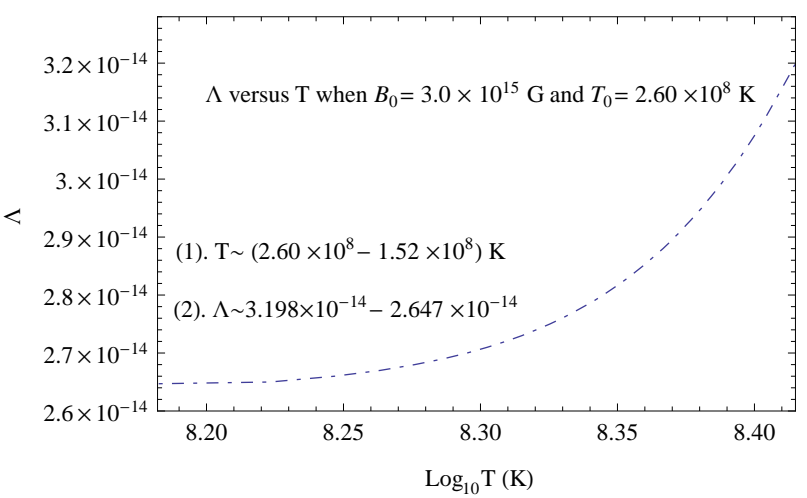

Fig. $2 \Lambda$ as a function of internal temperature $T$. The range of $T$ is about $\left(2.60 \times 10^{8}-1.52 \times 10^{8}\right) \mathrm{K}$ when $B_{0}=$ $3.0 \times 10^{15} \mathrm{G}$.

Using Eq.(7), we obtain the expression of $\Gamma$ in superhigh magnetic fields,

$$
\begin{aligned}
& \Gamma=\Lambda(B, T) \frac{2 \pi}{\hbar} G_{\mathrm{F}}^{2} C_{\mathrm{V}}^{2}\left(1+3 a^{2}\right) \int_{Q}^{E_{F}(\mathrm{e})} f_{\mathrm{e}}\left(1-f_{\nu}\right) \\
& \times f_{\mathrm{p}}\left(1-f_{\mathrm{n}}\right) \rho_{\nu} \rho_{\mathrm{e}} d E_{\mathrm{e}} \rho_{\nu} d E_{\nu} \delta\left(E_{\nu}+Q-E_{\mathrm{e}}\right) \\
& =\Lambda \frac{2 \pi G_{F}^{2} C_{V}^{2}\left(1+3 a^{2}\right)}{\hbar\left(2 \pi^{2} \hbar^{3} c^{3}\right)^{2} 3 m_{\mathrm{e}} c^{2}} \frac{1}{B^{*}} \int_{Q}^{E_{F}(\mathrm{e})} f_{\mathrm{e}}\left(1-f_{\nu}\right) f_{\mathrm{p}} \\
& \left.\times\left(1-f_{\mathrm{n}}\right)\left[E_{F}^{2}(\mathrm{e})-0.261-E_{\mathrm{e}}^{2}\right]^{\frac{3}{2}}\left(E_{\mathrm{e}}-Q\right)^{2}\right) d E,(8)
\end{aligned}
$$

where $f(\mathrm{j})=\left[\operatorname{Exp}\left(\left(E_{\mathrm{j}}-\mu_{\mathrm{j}}\right) / k T\right)+1\right]^{-1}$ is the fraction of phase space occupied at energy $E_{\mathrm{j}}$ (Fermi-Dirac distribution), factors of $\left(1-f_{\mathrm{j}}\right)$ reduce the reaction rate, and are called 'blocking factor', inside a NS, for neutrinos(antineutrinos), $\left(1-f_{\nu}\right)=1$; for electrons, when $E_{\mathrm{e}}<E_{\mathrm{F}}(\mathrm{e}), f_{\mathrm{e}}=1$, when $E_{\mathrm{e}}>E_{\mathrm{F}}(\mathrm{e}), f_{\mathrm{e}}=$ 0 ; for neutrons, when $E_{k}(\mathrm{n})<E_{\mathrm{F}}^{\prime}(\mathrm{n}),\left(1-f_{\mathrm{n}}\right)=0$, when $E_{k}(\mathrm{n})>E_{\mathrm{F}}^{\prime}(\mathrm{n}),\left(1-f_{\mathrm{n}}\right)=1$; for protons: when $E_{\mathrm{p}}<E_{\mathrm{F}}(\mathrm{p}), f_{\mathrm{p}}=1$, when $E_{\mathrm{p}}>E_{\mathrm{F}}(\mathrm{p}), f_{\mathrm{p}}=0$, so $f_{\mathrm{e}}\left(1-f_{\nu}\right) f_{\mathrm{p}}\left(1-f_{\mathrm{n}}\right) \simeq 1$ can be ignored in the latter calculations; the other quantities have been defined in Paper 1, Paper 2 and Paper 4. Eq.(6) is the very expression of $\Gamma$ we have been looking for. Compared with Eqs.(3-4) of Paper 2, the advantages of Eq.(6) mainly includes: (1) It reflects the effective or actual capture rates of electrons in superhigh magnetic fields, which can be calculated directly using Eq.(6), rather than be modified by parameter $q$; (2) It adopts the updated expressions of $\rho_{\mathrm{e}}$ and $E_{\mathrm{F}}(\mathrm{e})$ in superhigh magnetic fields, both of which are derived in circular cylindrical coordinates. In order to compare the results of $\Gamma$ in superhigh magnetic fields calculated by two different types of electron energy state density, we appeal to the following 


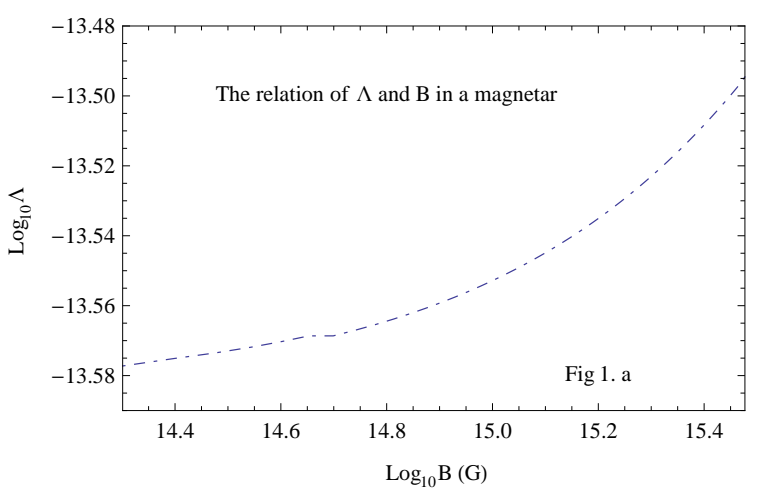

(a)

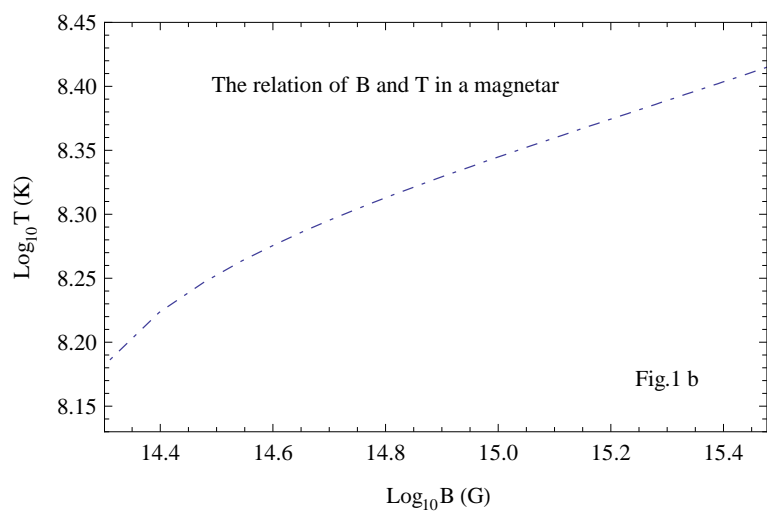

(b)

Fig. $1 \Lambda$ as a function of magnetic field $B$. The range of $B$ is about $\left(3.0 \times 10^{15}-2.0 \times 10^{14}\right) \mathrm{G}$ when $T_{0}=2.60 \times 10^{8} \mathrm{~K}$.

expression,

$$
\begin{aligned}
& \Gamma^{\prime}=\Lambda(B, T) \frac{2 \pi}{\hbar} G_{\mathrm{F}}^{2} C_{\mathrm{V}}^{2}\left(1+3 a^{2}\right) \int_{Q}^{E_{\mathrm{F}}(\mathrm{e})} f_{\mathrm{e}}\left(1-f_{\nu}\right) \\
& \times f_{\mathrm{p}}\left(1-f_{\mathrm{n}}\right) \rho_{\mathrm{e}} d E_{\mathrm{e}}^{\prime} \rho_{\nu} d E_{\nu} \delta\left(E_{\nu}+Q-E_{\mathrm{e}}\right) \\
& =\Lambda \frac{2 \pi}{\hbar} \frac{G_{\mathrm{F}}^{2} C_{\mathrm{V}}^{2}\left(1+3 a^{2}\right)}{\left(2 \pi^{2} \hbar^{3} c^{3}\right)^{2}} \int_{Q}^{E_{\mathrm{F}}(e)} f_{\mathrm{e}}\left(1-f_{\nu}\right) \\
& \times f_{\mathrm{p}}\left(1-f_{\mathrm{n}}\right)\left(E_{\mathrm{e}}^{2}-m_{\mathrm{e}}^{2} c^{4}\right)^{\frac{1}{2}} E_{\mathrm{e}}\left(E_{\mathrm{e}}-Q\right)^{2} d E_{\mathrm{e}}, \quad(9)
\end{aligned}
$$

where the most common and typical expression for electron energy state density $\rho_{\mathrm{e}}^{\prime}$ in a sphere symmetrical momentum space, $\rho_{\mathrm{e}}^{\prime}=\frac{4 \pi p_{\mathrm{e}}^{2}}{h^{3}} \frac{d p_{\mathrm{e}}}{d E_{\mathrm{e}}}=\frac{4 \pi p_{\mathrm{e}} E_{\mathrm{e}}}{c^{2} h^{3}}$, is used. Inserting $\frac{2 \pi}{\hbar} \frac{G_{\mathrm{F}}^{2} C_{\mathrm{V}}^{2}\left(1+3 a^{2}\right)}{\left(2 \pi^{2} \hbar^{3} c^{3}\right)^{2}}=0.018(\mathrm{MeV})^{-5} \mathrm{~s}^{-1}$ and $m_{\mathrm{e}} c^{2}=0.511 \mathrm{MeV}$ into Eq.(8) and Eq.(9), we obtain the values of electron capture rate in superhigh magnetic fields. The calculation results are partly listed below in tabular form. The main results in Table 1 are summarized as follows: When the magnetic field $B \sim\left(3.0 \times 10^{15}-1.8 \times 10^{14}\right) \mathrm{G}, \Gamma \sim\left(1.38 \times 10^{-7}-\right.$ $\left.2.43 \times 10^{-13}\right) \mathrm{s}^{-1}$ and $\Gamma^{\prime} \sim\left(6.52 \times 10^{-7}-7.76 \times 10^{-12}\right) \mathrm{s}$ ${ }^{-1}$, respectively. From Table 1 , the values of $\Gamma$ are universally less than those of $\Gamma^{\prime}$, and the ratio of $K=\Gamma / \Gamma^{\prime}$ is about the magnitude of $10^{-1}-10^{-2}$. The possible explanations are as follows:

1. Due to the formation of Landau cylinder in the momentum space or the quantization of Landau levels, the spherical symmetry in the momentum space is broken by superhigh magnetic fields. The formula of $\rho_{\mathrm{e}}$ and that of $\rho_{\mathrm{e}}^{\prime}$ are derived in circular cylindrical coordinates and in spherical coordinates, respectively.

2. In the vicinity of the Fermi surface, the electrons with the same energy $E$ could come from different Landau levels because the electrons are degenerate. However, the electrons occupying lower Landau levels cannot be captured even if their energies are higher than $Q$; for higher Landau levels, there still exist some electrons with lower energies $E(E<Q)$ that are not captured. Thus, the values of $\Gamma$ calculated by the expression of $\rho_{\mathrm{e}}$ in superhigh magnetic fields are less than those of $\Gamma^{\prime}$ computed by the expression of $\rho_{\mathrm{e}}^{\prime}$ in non-relativistic magnetic fields.

3. In Papers 1-2, in order to obtain the values of the effective electron capture rates $\Gamma_{\text {eff }}$ in superhigh magnetic fields, we introduce the Landau level effect coefficient $q$. In our improved model, the following factors: neutron superfluid's restraining effect, the numbers of all particles participating in EC reaction, thermal energy loss, energy conversion efficiency and so on, have been considered, so the quantity $q$ must be replaced by two important parameters: $\Lambda$ and $\zeta$ (see Paper 4 ). Actually, the quantity $q$ includes the effects of $\Lambda$ and $\zeta$. Thus, the values of $K$ is far less than those of $q$ (see Papers 1-2).

From Table 1, we obtain the diagram of $K$ as a function of $B$, shown as in Fig.3. Furthermore, the analytic

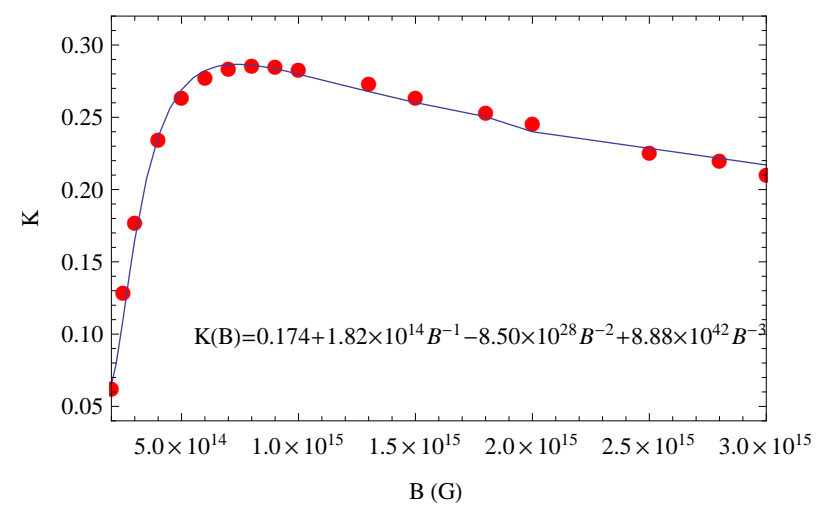

Fig. 3 The diagram of $K$ versus $B$ 
Table 1 The calculated values of electron capture rates in superhigh magnetic fields

\begin{tabular}{crrrrrrr}
\hline $\begin{array}{c}B \\
(\mathrm{G})\end{array}$ & $\begin{array}{r}E_{\mathrm{F}}(\mathrm{e}) \\
(\mathrm{MeV})\end{array}$ & $\begin{array}{r}E_{\mathrm{F}}(\nu) \\
(\mathrm{MeV})\end{array}$ & $\begin{array}{r}\left\langle E_{\mathrm{n}}\right\rangle \\
(\mathrm{MeV})\end{array}$ & $\begin{array}{r}\left\langle E_{\nu}\right\rangle \\
(\mathrm{MeV})\end{array}$ & $\begin{array}{r}\Gamma \\
\left(\mathrm{s}^{-1}\right)\end{array}$ & $\begin{array}{c}\Gamma^{\prime} \\
\left(\mathrm{s}^{-1}\right)\end{array}$ & $K=\Gamma / \Gamma^{\prime}$ \\
\hline $1.8 \times 10^{14}$ & 61.73 & 2.34 & 61.06 & 1.28 & $2.43 \times 10^{-13}$ & $7.76 \times 10^{-12}$ & $3.14 \times 10^{-2}$ \\
$2.0 \times 10^{14}$ & 63.38 & 3.99 & 61.81 & 2.18 & $2.50 \times 10^{-12}$ & $4.01 \times 10^{-11}$ & $6.24 \times 10^{-2}$ \\
$2.5 \times 10^{14}$ & 67.01 & 7.62 & 63.44 & 4.18 & $3.95 \times 10^{-11}$ & $3.06 \times 10^{-10}$ & $1.29 \times 10^{-1}$ \\
$3.0 \times 10^{14}$ & 70.14 & 10.75 & 64.84 & 5.91 & $1.64 \times 10^{-10}$ & $9.24 \times 10^{-10}$ & $1.78 \times 10^{-1}$ \\
$4.0 \times 10^{14}$ & 75.37 & 15.98 & 67.16 & 8.82 & $8.07 \times 10^{-10}$ & $3.43 \times 10^{-9}$ & $2.35 \times 10^{-1}$ \\
$5.0 \times 10^{14}$ & 79.69 & 20.30 & 69.07 & 11.23 & $2.04 \times 10^{-9}$ & $7.74 \times 10^{-9}$ & $2.63 \times 10^{-1}$ \\
$6.0 \times 10^{14}$ & 83.41 & 24.02 & 70.71 & 13.31 & $3.87 \times 10^{-9}$ & $1.39 \times 10^{-8}$ & $2.78 \times 10^{-1}$ \\
$7.0 \times 10^{14}$ & 86.69 & 27.30 & 72.15 & 15.15 & $6.24 \times 10^{-9}$ & $2.19 \times 10^{-8}$ & $2.84 \times 10^{-1}$ \\
$8.0 \times 10^{14}$ & 89.63 & 30.24 & 73.43 & 16.81 & $9.08 \times 10^{-9}$ & $3.17 \times 10^{-8}$ & $2.86 \times 10^{-1}$ \\
$9.0 \times 10^{14}$ & 92.31 & 32.92 & 74.60 & 18.32 & $1.24 \times 10^{-8}$ & $4.33 \times 10^{-8}$ & $2.85 \times 10^{-1}$ \\
$1.0 \times 10^{15}$ & 94.77 & 35.38 & 75.67 & 19.71 & $1.60 \times 10^{-8}$ & $5.61 \times 10^{-8}$ & $2.83 \times 10^{-1}$ \\
$1.5 \times 10^{15}$ & 104.88 & 45.49 & 80.05 & 25.44 & $3.90 \times 10^{-8}$ & $1.48 \times 10^{-7}$ & $2.64 \times 10^{-1}$ \\
$2.0 \times 10^{15}$ & 112.70 & 53.31 & 83.43 & 29.88 & $6.78 \times 10^{-8}$ & $2.78 \times 10^{-7}$ & $2.43 \times 10^{-1}$ \\
$2.5 \times 10^{15}$ & 119.17 & 59.78 & 86.21 & 33.57 & $1.01 \times 10^{-7}$ & $4.47 \times 10^{-7}$ & $2.26 \times 10^{-1}$ \\
$2.8 \times 10^{15}$ & 122.59 & 63.20 & 87.67 & 35.52 & $1.22 \times 10^{-7}$ & $5.56 \times 10^{-7}$ & $2.20 \times 10^{-1}$ \\
$3.0 \times 10^{15}$ & 124.72 & 65.33 & 88.59 & 36.74 & $1.38 \times 10^{-7}$ & $6.52 \times 10^{-7}$ & $2.11 \times 10^{-1}$ \\
\hline
\end{tabular}

expression of $K$ and $B$ is obtained by fitting the data of Table 1 ,

$K(B)=0.174+\frac{1.82 \times 10^{14}}{B}-\frac{8.50 \times 10^{28}}{B^{2}}+\frac{8.88 \times 10^{42}}{B^{3}}$,

From the definition of $K=\Gamma / \Gamma^{\prime}$, it is obvious that the value of $K$ is determined by $B$, and is irrelevant to $T$.

Since the updated expression of $\rho_{\mathrm{e}}$ is utilized, the values of $\Gamma$ are universally lower than those of $\Gamma^{\prime}$ slightly, however, we cannot differentiating Eq.(8) directly. To the contrary, Eq.(9) is very useful in differential calculations, especially in calculating magnetic fields' evolutionary timescales. In order to obtain a second-order differential equation of $B$ and $t$, we may combing Eq.(8) with Eq.(9). By using Eq.(10), we obtain an approximation relation between $\Gamma$ and $\Gamma^{\prime}$,

$$
\begin{aligned}
& \Gamma=K(B) \Gamma^{\prime}=0.018 K(B) \Lambda(B, T) \\
& \times \int_{Q}^{E_{\mathrm{F}}(\mathrm{e})}\left(E_{\mathrm{e}}^{2}-0.261\right)^{\frac{1}{2}} E_{\mathrm{e}}\left(E_{\mathrm{e}}-Q\right)^{2} d E_{\mathrm{e}} .
\end{aligned}
$$

\section{Magnetic field decay of magnetars in SNRs}

\subsection{Superhigh magnetic fields and their evolutionary timescales}

In order to investigate the whole process of the decay of superhigh magnetic fields, to begin with, let us make two approximations: (1) A magnetar can be treated as a common NS with a total mass of $M=2.8 \times 10^{33} \mathrm{~g}$ (that is about 1.4 times the solar mass) and a radius of
$R=10^{6} \mathrm{~cm}$; (2) The whole electron capture timescale is equal to the decay timescale of superhigh magnetic fields (without consideration of the modified Urca process).

As discussed in Sec.1, if the ${ }^{3} P_{2}$ neutron Cooper pairs are destroyed by the outgoing EC neutrons, both the anisotropic superfluid and superhigh magnetic fields produced by the aligned magnetic moments of the ${ }^{3} P_{2}$ Cooper pairs will disappear gradually. Employing Eq.(10) can allow us to gain a differential equation

$$
\begin{aligned}
& \frac{d \Gamma}{d t} \approx K(B) \frac{d \Gamma^{\prime}}{d t}=0.018 K(B) \Lambda(B, T)\left(E_{\mathrm{F}}(\mathrm{e})-Q\right)^{2} \\
& E_{\mathrm{F}}(\mathrm{e})\left(E_{\mathrm{F}}^{2}(\mathrm{e})-m_{\mathrm{e}}^{2} c^{4}\right)^{\frac{1}{2}} 43.44 \times \frac{1}{4} B^{-\frac{3}{4}} B_{\mathrm{cr}}^{-\frac{1}{4}} \frac{d B}{d t},(12)
\end{aligned}
$$

where $\frac{d(0.018 K(B) \Lambda(B, T))}{d t}$ is ignored because of its too low value $\left(0.018 K(B) \Lambda(B, T) \sim 10^{-17} \sim 10^{-18}(\mathrm{MeV})\right.$ $-5 \mathrm{~s}^{-1}, \frac{d(0.018 K(B) \Lambda(B, T)}{d t} \sim \frac{\Delta(0.018 K(B) \Lambda(B, T)}{\Delta t} \sim$ $\frac{10^{-17} \sim 10^{-18}(\mathrm{MeV})^{-5} \mathrm{~s}^{-1}}{10^{13} \mathrm{~s}} \sim 10^{-30} \sim 10^{-31}(\mathrm{MeV})^{-5} \mathrm{~s}^{-2}$, the integral term $\int_{Q}^{E_{\mathrm{F}}(\mathrm{e})}\left(E_{\mathrm{e}}^{2}-0.261\right)^{\frac{1}{2}} E_{\mathrm{e}}\left(E_{\mathrm{e}}-Q\right)^{2} d E_{\mathrm{e}} \sim$ $10^{10}-10^{6}(\mathrm{MeV})^{5}$, and its time derivative $\sim 10^{-3}-$ $10^{7}(\mathrm{MeV})^{5} \mathrm{~s}^{-1}$ assuming $\left.\Delta t \sim 10^{6} \mathrm{yrs}\right)$. Using binomial expansion theorem, the term $\left(E_{\mathrm{F}}^{2}(\mathrm{e})-m_{\mathrm{e}}^{2} c^{4}\right)^{\frac{1}{2}}$ can be expanded as:

$$
\begin{aligned}
& \left(E_{\mathrm{F}}^{2}(e)-m_{\mathrm{e}}^{2} c^{4}\right)^{\frac{1}{2}}=E_{\mathrm{F}}(e)\left(1-m_{\mathrm{e}}^{2} c^{4} / 2 E_{\mathrm{F}}^{2}(e)\right. \\
& \left.-m_{\mathrm{e}}^{4} c^{8} / 8 E_{\mathrm{F}}^{4}(e)+\cdots\right) \approx 43.44\left(\frac{B}{B_{\mathrm{cr}}}\right)^{\frac{1}{4}} \\
& \times\left(1-542 B^{-\frac{1}{2}}-146932 B^{-1}+\cdots\right) .
\end{aligned}
$$

Since $542 B^{-\frac{1}{2}} \sim 10^{-5}$ and $146932 B^{-1} \sim 10^{-10}$, we will reserve the first term in the bracket of the binomial expansion in the following calculations. Since 
a normal radio pulsar can be treated as a system of magnetic dipoles, there is an approximation relation of $\mu=\frac{1}{2} B R_{6}^{3}$, where $\mu, B$ and $R_{6}$ are the dipole magnetic moment, the dipole magnetic field strength and the radius of the star in units of $10^{6} \mathrm{~cm}$, respectively (Shapiro \& Teukolsky 1983). Like normal radio pulsars, a magnetar can be seen as a magnetic dipole system, the above approximation relation is also hold in a magnetar. Assuming that one outgoing EC neutron can destroy one ${ }^{3} P_{2}$ Cooper pair (see Appendix A), the decay rates of magnetic fields of magnetars can be estimated as

$\frac{d B}{d t}=\frac{2}{R_{6}^{3}} \frac{d \mu}{d t}=\frac{2}{R_{6}^{3}}\left(-\Gamma 2 \mu_{\mathrm{n}} n_{\mathrm{e}} V\left({ }^{3} P_{2}\right)\right)$,

where $V\left({ }^{3} P_{2}\right)$ denotes the volume of the ${ }^{3} P_{2}$ anisotropic neutron superfluid, $V\left({ }^{3} P_{2}\right)=\frac{4}{3} \pi R_{5}^{3} \mathrm{~cm}^{3}, R_{5}=10^{5} \mathrm{~cm}$, and $n_{\mathrm{p}}=n_{\mathrm{e}}=9.6 \times 10^{35} \mathrm{~cm}^{-3}$ setting $\rho=\rho_{0}$. Since $\Gamma$ in this paper represents the effective electron capture rate, Eq.(14) deviates greatly from Eq.(11) in Paper 2, though they are exactly like. Be note, in the interior of a NS, the processes of EC and $\beta$-decay exist at the same time, which is required by electric neutrality, the depleted protons/electrons are recycled for many times, so the alteration of $Y_{\mathrm{p}} / Y_{\mathrm{e}}$ could be very small. From Eq.(14), we get

$\frac{d \Gamma}{d t}=\frac{-R_{6}^{3}}{4 \mu_{\mathrm{n}} n_{\mathrm{e}} V\left({ }^{3} P_{2}\right)} \frac{d^{2} B}{d t^{2}}$,

where $\mu_{n}=0.966 \times 10^{-23}$ erg $\mathrm{G}^{-1}$ is the absolute value of the neutron abnormal magnetic moment. Combining Eq.(12) with Eq.(15) and eliminating $\Gamma$ yields a secondorder differential equation:

$$
\begin{aligned}
& \frac{d^{2} B}{d t^{2}}+4.8002 \times 10^{24}\left(2.60388 \times 10^{-14}+1.98233\right. \\
& \left.\times 10^{-30} B\right)\left(0.174+1.82 \times 10^{14} B^{-1}-8.50 \times 10^{28}\right. \\
& \left.B^{-2}+8.88 \times 10^{42} B^{-3}\right)\left(1.98 \times 10^{-25} B^{\frac{1}{4}}-1.394\right. \\
& \left.\times 10^{-21}+2.458 \times 10^{-18} B^{-\frac{1}{4}}\right) \frac{d B}{d t}=0
\end{aligned}
$$

where $T_{0}=2.6 \times 10^{8} \mathrm{~K}$ is used. For the purpose of calculating the whole electron capture time $t$, we can treat this second-order differential equation as follows: Firstly, decreasing the order of Eq.(16) gives a first- order differential equation

$$
\begin{aligned}
& \frac{d B}{d t}=-\left(-1.2125 \times 10^{36} B^{\frac{-9}{4}}+7.73615 \times 10^{32}\right. \\
& B^{-2}-1.2558 \times 10^{29} B^{\frac{-7}{4}}+2.0725 \times 10^{22} B^{\frac{-5}{4}}- \\
& 1.46924 \times 10^{19} B^{-1}+2.78251 \times 10^{15} B^{\frac{-3}{4}}-2.1571 \\
& \times 10^{8} B^{\frac{-1}{4}}+17.3762 B^{\frac{1}{4}}+7.69529 \times 10^{-8} B^{\frac{3}{4}} \\
& -3.27316 \times 10^{-11} B-30.5838 \log _{10} B+3.7193 \\
& -1.15403 \times \times 10^{-15} B^{\frac{5}{4}}+2.32557 \times 10^{-24} B^{\frac{7}{4}} \\
& \left.10^{-27} B^{2}+1.45703 \times 10^{-31} B^{\frac{9}{4}}\right)+C .
\end{aligned}
$$

Secondly, inserting the boundary condition: $d B / d t=0$ when $B=B_{\text {th }}$, into Eq.(17) determines the constant of integral $C=-992890.0$; Thirdly, integrating over $B$ gives a general expression of $t$

$$
\begin{aligned}
& t=\int_{B_{\mathrm{i}}}^{B_{\mathrm{f}}}-\left(-1.2125 \times 10^{36} B^{\frac{-9}{4}}+7.73615 \times 10^{32}\right. \\
& B^{-2}-1.2558 \times 10^{29} B^{\frac{-7}{4}}+2.0725 \times 10^{22} B^{\frac{-5}{4}}- \\
& 1.46924 \times 10^{19} B^{-1}+2.78251 \times 10^{15} B^{\frac{-3}{4}}-2.1571 \\
& \times 10^{8} B^{\frac{-1}{4}}+17.3762 B^{\frac{1}{4}}+.69529 \times 10^{-8} B^{\frac{3}{4}} \\
& -3.27316 \times 10^{-11} B-30.5838 \log _{10} B+3.7193 \times \\
& 10^{-15} B^{\frac{5}{4}}+2.32557 \times 10^{-24} B^{\frac{7}{4}}-1.15403 \times 10^{-27} \\
& \left.B^{2}+1.45703 \times 10^{-31} B^{\frac{9}{4}}+992890.0\right)^{-1} d B .
\end{aligned}
$$

Finally, using integral transform $B^{\frac{1}{4}} \rightarrow x$ and $d B \rightarrow$ $4 x^{3} d x$ gives the final expression of $t$

$$
\begin{aligned}
& t=\int_{B_{\mathbf{f}}^{0.25}}^{B_{\mathbf{i}}^{0.25}}\left(-1.2125 \times 10^{36} x^{-9}+7.73615 x^{-8}-\right. \\
& 1.2558 \times 10^{29} x^{-7}+2.0725 \times 10^{22} x^{-5}-1.46924 \\
& \times 10^{19} x^{4}+2.78251 \times 10^{15} x^{-3}-2.1571 \\
& \times 10^{8} x^{-1}+17.3762 x+7.69529 \times 10^{-8} x^{3} \\
& -3.27316 \times 10^{-11} x^{4}-30.5838 \log _{10} x^{4}+3.7193 \times \\
& 10^{-15} x^{5}+2.32557 \times 10^{-24} x^{7}-1.15403 \times 10^{-27} \\
& \left.x^{8}+1.45703 \times 10^{-31} x^{9}+992890.0\right)^{-1} 4 x^{3} d x,
\end{aligned}
$$

where $x \geq B_{\mathrm{th}}^{0.25}=3524.05$. In order to investigate the characteristics of Eq.(19), we introduce a variable $F(x)$ to denote the integrated function, $F(x)=(-1.2125 \times$ $10^{36} x^{-9}+7.73615 \times 10^{32} x^{-8}-1.2558 \times 10^{29} x^{-7}+$ $2.0725 \times 10^{22} x^{-5}-1.46924 \times 10^{19} x^{-4}+2.78251 \times$ $10^{15} x^{-3}-2.1571 \times 10^{8} x^{-1}+17.3762 x+7.69529 \times$ $10^{-8} x^{3}-3.27316 \times 10^{-11} x^{4}-30.5838 \log _{10} x^{4}+3.7193 \times$ $10^{-15} x^{5}+2.32557 \times 10^{-24} x^{7}-1.15403 \times 10^{-27} x^{8}+$ $\left.1.45703 \times 10^{-31} x^{9}+992890.0\right)^{-1} 4 x^{3}$, and make a schematic diagram of $F(x)$ as a function of $x$, shown as in Fig.4. From Fig.4, in the integral interval of $\left[\left(x_{1} \sim x_{2}\right), x_{1} \leq x_{2}\right]$, the function $F(x)$ is convergent, 


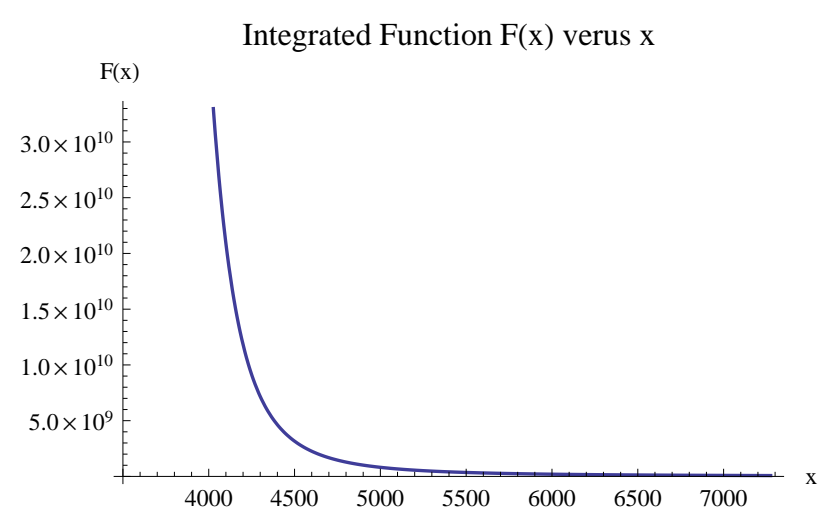

Fig. 4 The diagram of $F(x)$ versus $x$

and hence is integrable, where $x=3524.05$ is a singularity of $F(x)$. By solving Eq.(19), we gain the whole electron capture time (or the superhigh magnetic field's decay timescale), $t \approx 9.2947 \times 10^{13} \mathrm{~s}=2.9507 \times 10^{6}$ yrs when $B_{\mathrm{i}}=3.0 \times 10^{15} \mathrm{G}$ and $B_{\mathrm{f}}=B_{\mathrm{th}}$; in the same way, if $B_{\mathrm{i}}=3.0 \times 10^{15} \mathrm{G}$ and $B_{\mathrm{f}}=4.0 \times 10^{14} \mathrm{G}$, $t \approx 1.4737 \times 10^{12} \mathrm{~s}=4.6785 \times 10^{4} \mathrm{yrs}$, corresponding to $L_{\mathrm{X}} \sim\left(10^{37} \sim 10^{34}\right) \mathrm{erg} \mathrm{s}^{-1}$. Furthermore, the fitting curves of $\log _{10} B$ versus $\log _{10} t$ for different magnetic field ranges are shown in Fig.5.

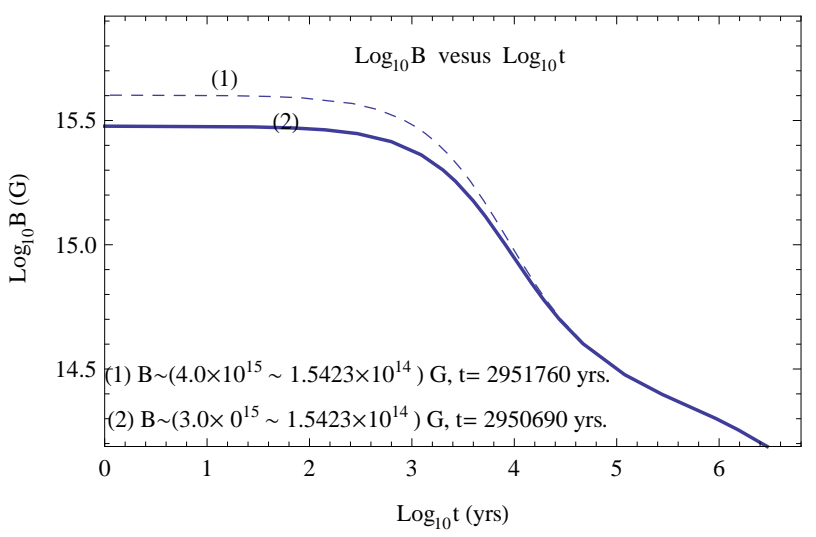

Fig. 5 Superhigh magnetic field $B$ as a function of time $t$.

From Fig.5, the magnetic field decreases with increasing time obviously. Customarily, $B=3.0 \times 10^{15}$ $\mathrm{G}$ is assumed to be the possible initial magnetic field, while $B=4.0 \times 10^{15} \mathrm{G}$ is assumed to be the upper limit of magnetic field of a magnetar by some authors (e.g., recent papers of Peng \& Tong 2007, 2009, and Gao et al 2011). However, the difference between the evolution timescales of these two fields is only $\Delta t \simeq 1070 \mathrm{yrs}$, obtained from Eq.(19) (as shown in Fig.5), which implies a substantial positive correlation between the magnetic field and its decay rate.
Since the initial value of internal temperature $T$ is far higher than its current value for a magnetar, we arbitrarily assume $T_{0} \sim 2.75 \times 10^{8}-1.75 \times 10^{8} \mathrm{~K}$ within a permitted and plausible temperature range under our magnetar model. Repeating all the calculations above gives the relation of $t$ and $T_{0}$, shown as in Fig.6.

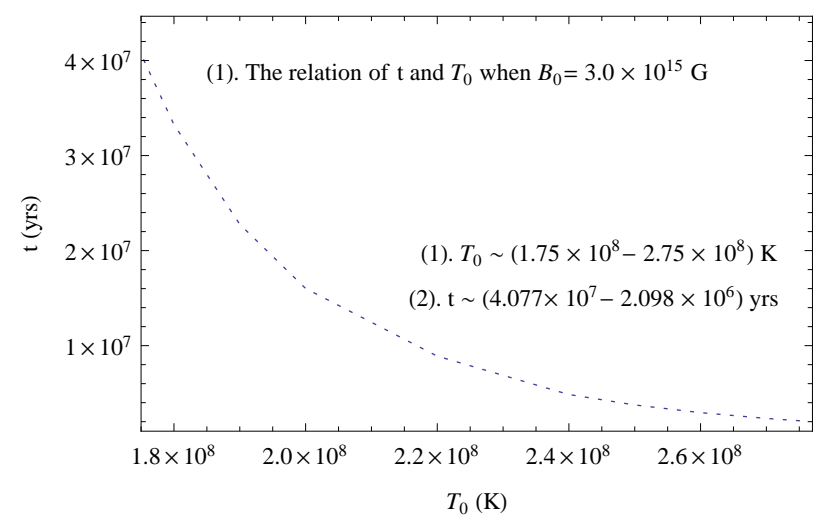

Fig. 6 The magnetic field evolution timescale $t$ as a function of time $T_{0}$.

From Fig.6, it is apparent that the lower $T_{0}$, the higher $t$ if $B_{0}$ is invariable. When $B_{0}=3.0 \times 10^{15}$ $\mathrm{G}, T_{0} \sim 2.75 \times 10^{8}-1.75 \times 10^{8} \mathrm{~K}$, then $t \sim 2.098 \times$ $10^{6}-4.077 \times 10^{7}$ yrs.

\subsection{The initial internal fields of magnetar progenitors}

Although we have presented a reasonable explanation for the origin of superhigh magnetic fields in the previous work (Peng \& Tong 2007; Peng \& Tong 2009), many issues concerning magnetars remain unsolved. So far, the magnetic fields of magnetars obtained from the observations are just their surface dipolar magnetic fields $B_{\text {dip }}$, assuming a simple magnetic dipole spindown model. What is the relationship between the surface dipolar magnetic field and the internal magnetic field in a magnetar? How strong is the initial internal magnetic field $B_{\mathrm{i}}$ of a magnetar progenitor? How long will such an intense field $\left(B_{\mathrm{i}}\right)$ continue to decay? What will eventually happen in the interior of a magnetar when $B$ drops below $B_{\mathrm{th}}$ ? All these questions are all very basic, and remain open.

In this part, motivated by SNR associations, we try to carry out the studies of $B_{\mathrm{i}}$ for magnetar progenitors. Observations indicate that SNRs have been expanding, and interacting with their surroundings since the supernova explosions. Therefore, the ages of SNRs may be computed by modeling their morphologies at the current epoch. The true ages of magnetars obtained 
Table 2 The calculated values of the initial internal magnetic fields of magnetar progenitors

\begin{tabular}{crrrrrrr}
\hline Source & $P$ & $\dot{P}$ & $B_{\text {dip }}$ & SNR & $t_{\text {SNR }}$ & $B_{i}^{a}$ & Ref $^{d}$ \\
\hline SGR 0526-66 & 8.0544 & 3.8 & 5.6 & N49 & 5.0 & 5.06 & {$[1,2]$} \\
SGR 1806-20 & 7.6022 & 75 & 24 & G10.0-0.3 & $<10^{\ddagger}$ & $\leq 29.3$ & {$[3,4]$} \\
SGR 1627-41 & 2.5946 & 1.9 & 2.20 & G33.70-01 & 5.0 & 2.204 & {$[5,6]$} \\
SGR 1900+14 & 5.1998 & 9.2 & 7.0 & G42.8+0.6 & $<10^{\S}$ & $<13.71$ & {$[7,8]$} \\
1E 2259+586 & 6.9789 & 0.048 & 0.59 & CTP109 & $\sim 10$ & $\sim 1.5435$ & {$[9,10]$} \\
1E 1841-045 & 11.7829 & 3.93 & 6.9 & Kes73 & 2 & 7.497 & {$[11,12]$} \\
1E 1547.0-5408 & 2.318 & 2.318 & 2.2 & G327.24-0.13 & $<1.4$ & $<2.202$ & {$[13,14]$} \\
CXOU J171405.7 & 3.82535 & 6.40 & 5.0 & CTB37B & 4.9 & 5.509 & {$[15-17]$} \\
AX J1845-0258 & 6.97127 & No & No & G29.6+0.1 & $<8$ & $\leq 40^{b}$ & {$[18,19]$} \\
\hline
\end{tabular}

Note: The units of period $P$, period derivative $\dot{P}$, the surface dipolar magnetic field $B_{\mathrm{dip}}$, SNR's age $t_{\mathrm{SNR}}$ and the initial internal magnetic field $B_{\mathrm{i}}$ are $\mathrm{s}, 10^{-11} \mathrm{~s} \mathrm{~s}^{-1}, 10^{14} \mathrm{G}, 10^{3}$ yrs and $10^{14} \mathrm{G}$, respectively.

${ }^{a}$ The values of the initial internal magnetic fields of magnetar progenitors $B_{\mathrm{i}}$ are gained by using Eq.(18) and $t \simeq t_{\mathrm{SNR}}$.

${ }^{b}$ Since AXP 1E 2259+586 could be associated with accretion (see Paper 4), and its present value of $B_{\text {dip }}$ is far less than $B_{\text {th }}$, its maximum value of $B_{\mathrm{i}}$ has to be estimated under our model.

${ }^{c}$ Since some important parameters (eg., $\dot{P}, B_{\text {dip }}$, the soft X-ray luminosity $L_{\mathrm{X}}$, and so on) of magnetar candidate AX J1845-0258 are uncertain, its maximum value of $B_{\mathrm{i}}$ has to be estimated under our model.

$d$ 1-(Kulkarni et al. 2003); 2-(Klose et al. 2004); 3-(Kulkarni \& Frail 1993); 4-(Marsden et al. 2001); 5-(Corbel et al. 1999); 6-(Wachter et al. 2004); 7-(Hurley et al. 1999); 8-(Mazets et al. 1999) 9-(Green 1989); 10-(Rho \& Petre 1997); 11-(Sanbonmatsu \& Herfand 1992); 12-(Vasisht \& Gotthelf 1997); 13-(Camilo et al. 2007); 14-(Gelfand \& Gaensler 2007); 15-(Aharonian et al. 2008); 16-(Halpern \& Gotthelf 2010); 17-(Horvath \& Allen 2011); 18-(Gaensler et al. 1999); 19- Vasisht et al. 2000).

$\dagger$ This candidate is unconfirmed.

$\ddagger$ Cited from Kulkani \& Frail(1993) and Marsden et al.(2001). § Cited from Hurley et al.(1999). All primitive data are from McGillAXP/SGR online catalog of 2 March 2012(http://www.physics mcgill.ca/ pulsar/magnetar/ main.html) and references cataloged.

from the ages of their SNRs are independent of the stars' properties, and thus basically unbiased even if AXPs or SGRs are strange stars (Zhang et al. 2000; $\mathrm{Xu}$ et al. 2006). Table 2 shows the data on 9 claimed magnetar-supernova remnant associations, which are cited from McGillAXP/SGR online catalog updated except for SGR 1806-20 and SGR 1900+14. As is known to us, all the known SNRs associated with common radio pulsars are very young, $t_{\mathrm{SNR}} \ll 10^{6}$ yrs. From Table 2, the ages of all the SNRs are not more than 10,000 yrs, which implies that the associated magnetars are more younger, compared with common radio pulsars. Perhaps these magnetars born with different physical properties (e.g., the equations of state, magnetic fields, inner temperatures, and so on) have experienced evolutionary routes that differ from those of common radio pulsars. For the sake of computing conveniently, we assume a simple magnetic dipole spin-down model, then the current magnetic field inside a magnetar is about its surface dipole field, i.e., $B_{\mathrm{f}} \simeq B_{\text {dip }}$ under this assumption. The field decay timescale of a magnetar equals approximately to the age of its $\mathrm{SNR}$, i.e., $t \simeq t_{\mathrm{SNR}}$, base on the results of ZX2011. Combining Eq.(19) with Table 2 gives the values of $B_{\mathrm{i}}$ for magnetar progenitors. The calculated results show that the values of $B_{\mathrm{i}}$ are concentrated primarily between $2.0 \times 10^{14} \mathrm{G}$ and $2.93 \times 10^{15} \mathrm{G}$ when $T_{0} \sim 2.60 \times 10^{8} \mathrm{~K}$. If $T_{0} \sim 2.75 \times 10^{8}-1.75 \times 10^{8} \mathrm{~K}$, there still be $B_{\mathrm{i}} \sim\left(10^{14}-10^{15}\right) \mathrm{G}$, not exceeding the upper limit of magnetic field of $4.0 \times 10^{15} \mathrm{G}$. The calculated results of $B_{\mathrm{i}}$ for magnetar progenitors illustrate that our magnetar model is consistent theoretically.

3.3 The spin-down ages of magnetars and the ages of their SNRs

As pointed out in Sec.1, the NS's spin-down age, also called the characteristic age, can be generally expressed as, $t_{\text {Spin }}=P /(n-1) \dot{P}$. In all kinds of catalog on pulsars, the spin-down ages of pulsars are usually evaluated by a simple magnetic dipole spin-down model, $t_{\text {Spin }}=P / 2 \dot{P}(n=3)$. In ZX2011, authors found that there is a strong and significant positive correlation between $\log _{10}(\operatorname{SNR}($ Age $) /$ Spindown(Age)) and $\log _{10} B$ via statistical analysis of radio pulsars associated with SNRs. They argued that, as NSs get older, their spin periods become longer duo to their spin-down torques, the decay of magnetic fields cause $\dot{P}$ to be far less than the mean values of $\dot{P}$ in history, their characteristic ages, inferred from parameters $P$ and $\dot{P}$ at the current epoch, will be larger than their real ages, denoted as $t_{\text {Real }}$. In a word, it's the dipolar magnetic field decay that plays a significant role in making a NS look older than it really is. 
In this part, we investigate the spin-down ages of magnetars and the ages of their SNRs, and made a diagram of $\log _{10}(\operatorname{SNR}($ Age $) /$ Spindown(Age)) versus $\log _{10} B$.

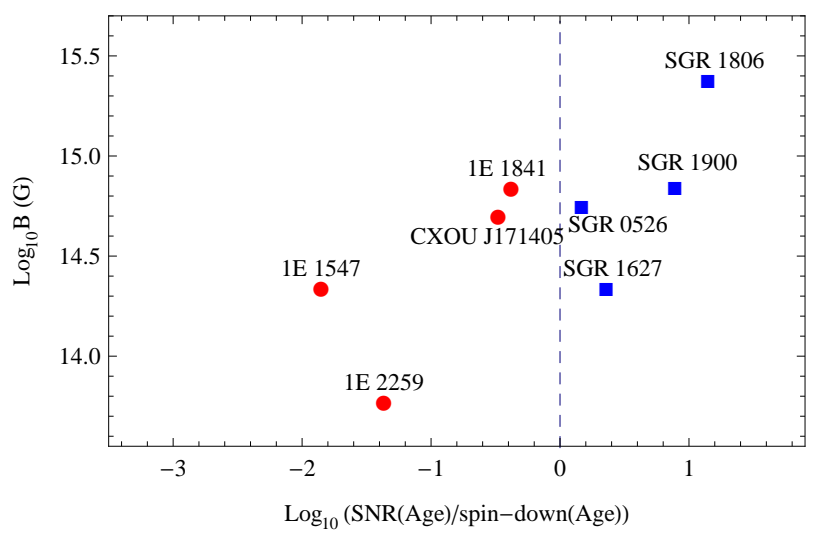

Fig. $7 \log _{10}(\operatorname{SNR}($ Age $) /$ Spindown(Age) $)$ versus $\log _{10} B$. The range of $B$ is $\left(1.5423 \times 10^{14} \sim 3.0 \times 10^{15}\right) \mathrm{G}$. The dashed line corresponds to $t_{\mathrm{SNR}}=t_{\mathrm{Spin}}$.

From Fig.7, an obvious correlation has been proved between $\log _{10} B$ and $\log _{10}(\mathrm{SNR}$ (Age)/Spindown(Age) for magnetars associated with SNRs. It is worthwhile to note that all AXPs associated with SNRs are on the left of the dashed line whereas all SGRs associated with SNRs are on the left of the line. The causes of this will be discussed at length in the following.

Since a magnetar can be seen as a magnetic dipole system, the above suggestion that 'the dipolar magnetic field decay plays a significant role in making a NS look older' is also applicable to magnetars. For all the AXPs (including candidates) with dipole magnetic fields $B<7.0 \times 10^{14} \mathrm{G}$, there have been no super-bursts or giant flares, at which huge energies $\left(\sim 10^{43}-10^{47} \mathrm{erg}\right)$ are suddenly released. This suggests that all AXPs could have experienced relatively 'normal' decay of their dipole magnetic fields compared with SGRs associated with SNRs, and thus have lower braking indexes, $n<3$.

In previous works, many authors proposed various models to explain why the observed braking index $n<3$, eg., neutrino and photon radiation coming from superfluid neutrons may brake the pulsars (Peng et al. 1982); both magnetic dipole radiation and the propeller torque applied by the debris disk may cause spindown of pulsars (Alpar et al. 2001; Menou et al. 2001); the combination of dipole radiation and the unipolar generator may cause $n$ decrease greatly $\mathrm{Xu} \& \mathrm{Qiao}$ 2001; Wu et al 2003); a variation of the torque function is important attribution for low braking index (Allen \& Horvath 1997); additional torques due to accretion may cause $n$ decrease Menou et al. 2001; Chen \& Li 2006); $n<3$ may be due to the decay of magnetic field strengthes(Blandford \& Romani 1988; Lin \& Zhang 2004; Chen \& Li 2006; Zhang \& Xie 2011), and so on. As we know, magnetars are high magnetized NSs. They universally possess very strong surface dipole magnetic fields $\left(\sim 10^{14}-10^{15} \mathrm{G}\right)$ with rare exceptions (eg., SGR 0418+5729, 1E 2259+586 and unconfirmed candidate Swift J1822.3-1606). Thus, we favor the braking model with changing magnetic field strengthes, ie., the decay of magnetic field leads to $n<3$ for magnetars. In our magnetar model, the lower braking indices $n(n<3)$ of AXPs are supposed to be correlated with the dipole magnetic fields $B$ and their decay rates $d B / d t$. In order to validate this assumption,, we investigate the phenomenon of $n<3$ for neutron stars (including common radio pulsars and magnetars) under pure magnetic dipole spin-down model, theoretically.

As we know, the spin frequency $\Omega$ of pulsars decreases with time, and the time derivative of $\Omega$ is proportional to some power of $\Omega$,

$I \dot{\Omega}=-K \Omega^{n}$

where $K=2 B^{2} R^{6} \sin ^{2} \theta / 3 c^{2}, \theta$ is the inclination of the magnetic axis with respect to the rotation axis; $B, R I$ are the surface magnetic field strength, the radius, and the momentum of inertia of the pulsar, respectively; $c$ is the velocity of light (Manchester \& Taylor 1977). The braking index $n$ of a pulsar can be a measured by differentiating Eq.(20),

$n=\frac{\ddot{\Omega} \Omega}{\dot{\Omega}^{2}}$,

where $\ddot{\Omega}$ is the second order time derivative of $\Omega$. In the model that assumes spin-down is due to pure magnetodipole radiation with a constant magnetic field, we obtain the ideal values of $n$ in Eqs.(20-21), $n=$ 3 (Manchester \& Tavlor 1977; Blandford \& Romani 1988; Menou et al. 2001; Chen \& Li 2006; Zhang \& Xie 2011). As mentioned in Sec.1, the observed values of $n$ from Eq.(21) always deviate from 3 expected for pure magnetodipole radiation model (only in this case, $\left.t_{\text {Spin }}=t_{\mathrm{SNR}}\right)$. With respect to the case of $n<3$, the main and possible causes have been provided, as listed above. In this paper, we will simply discuss the error(or deviation) of $n$ caused by the derivation of Eq.(21) itself. From the deduction above, Eq.(21) is obtained by differentiating Eq.(20), assuming $I$ and $K$ are constant. As a result, $n$ is mainly determined by $\Omega$ (or spin period $P, P=2 \pi / \Omega)$ and its time derivatives, and is irrelevant to the other quantities. Actually, it's possible that 
the quantities of $B, \theta, I, R$ and $\Omega$ change in varying degrees, and changes of $B, \theta, I$ and/or $R$ with $\Omega$ will induce a braking index $n \neq 3$. Hence, a modification of Eq.(21) is necessary. Keeping the quantities of $\theta, I$ and $R$ unchanged (the variances of these quantities are small usually), we re-differentiate Eq.(20) under pure magnetic dipole spin-down model, and get

$\ddot{\Omega}=\frac{d \dot{\Omega}}{d t}=\frac{-2 R^{6} \sin ^{2} \theta}{3 c^{2} I}\left(2 B \dot{B} \Omega^{3}+3 B^{2} \Omega^{2} \dot{\Omega}\right)$,

Inserting Eq.(20) and Eq.(22) into Eq.(21), we have

$$
\begin{aligned}
& n=\frac{-2 R^{6} \sin ^{2} \theta}{3 c^{2} I}\left(\frac{2 B \dot{B} \Omega^{4}}{\dot{\Omega}^{2}}+\frac{3 B^{2} \Omega^{3} \dot{\Omega}}{\dot{\Omega}^{2}}\right) \\
& =\frac{-2 R^{6} \sin ^{2} \theta}{3 c^{2} I}\left(\frac{9 c^{4} I^{2} \dot{B}}{2 R^{12} \sin ^{4} \theta \Omega^{2} B^{3}}-\frac{9 c^{2} I}{2 R^{6} \sin ^{2} \theta}\right) \\
& =3-\frac{3 c^{2} I \dot{B}}{R^{6} \sin ^{2} \theta \Omega^{2} B^{3}}=3-\frac{3 c^{2} P^{2} I}{4 \pi^{2} R^{6} \sin ^{2} \theta} \frac{\dot{B}}{B^{3}} \\
& =3-\frac{3 c^{2} P^{2} I}{4 \pi^{2} R^{6} \sin ^{2} \theta} \frac{|d B / d t|}{B^{3}}
\end{aligned}
$$

where $\dot{B}$ denotes the absolute value of decay rate of dipole magnetic field without considering any other torque (ie., the dipole magnetic field normally decays). We can estimate the magnitude of the second term in Eq.(23) as following: for a common radio pulsar, $I \sim 10^{45} \mathrm{~g} \mathrm{~cm}^{2}, \sin ^{2} \theta \sim 1, R \sim 10^{6} \mathrm{~cm}, P \sim 1 \mathrm{~s}$, $c=3.0 \times 10^{10} \mathrm{~m} \mathrm{~s}^{-1}, \frac{\dot{B}}{B^{3}} \sim \frac{|d B / d t|}{B^{3}} \sim \frac{|\Delta B / \Delta t|}{B^{3}} \sim$ $\frac{1}{B^{2} t} \sim 10^{-38}-10^{-39} \mathrm{G}^{-2} \mathrm{~s}^{-1}\left(\Delta B \sim B \sim 10^{12} \mathrm{G}\right.$, $\left.\Delta t \sim t \sim 10^{14}-10^{15} \mathrm{~s}\right), \frac{3 c^{2} P^{2} I}{4 \pi^{2} R^{6} \sin ^{2} \theta} \frac{\dot{B}}{B^{3}} \sim 10^{-8}-10^{-9}$; for a canonic magnetar, $P \sim 10 \mathrm{~s}, \frac{\dot{B}}{B^{3}} \sim \frac{1}{B^{2} t} \sim$ $10^{-41}-10^{-43} \mathrm{G}^{-2} \mathrm{~s}^{-1}\left(\Delta B \sim B \sim 10^{14}-10^{15}\right.$, $\left.\Delta t \sim t \sim 10^{13} \mathrm{~s}\right), \frac{3 c^{2} P^{2} I}{4 \pi^{2} R^{6} \sin ^{2} \theta} \frac{\dot{B}}{B^{3}} \sim 10^{-9}-10^{-10}$, therefore, when all the quantities of $\theta, I, R$ and $P$ (or $\Omega$ ) remain unchanged, and the dipole magnetic field normally decays, $n \approx 3$, in the ideal situation of $\dot{B}=0$, $n=3$. However, if there are substantial changes of $\theta$, $I, R$ and $P$ (at least one quanity varies), the effects of $\frac{\dot{B}}{B^{3}}$ on $n$ cannot be ignored. Under pure magnetic dipole spin-down model, we produce the diagrams of $d B / d t-B$ and $\frac{\dot{B}}{B^{3}}-B$ by using the method of curve fitting, shown as in Fig.8.

Fig. 8 is composed of four sub-figures. The fitted curves in Fig. 8 a and Fig. 8 b are obtained from Eq.(17). Fig. 8 a shows that $\frac{d B}{d t}$ decreases with decreasing $B$ significantly in the directed EC process. In Fig. $8 b, \frac{\dot{B}}{B^{3}}$ increases with decreasing $B$ when $B \sim 3.0 \times 10^{15} \sim$ $(4-5) \times 10^{14} \mathrm{G}$, because $B^{3}$ decreases faster than $\dot{B}$, whereas $\frac{\dot{B}}{B^{3}}$ decreases with decreasing $B$ when $B \sim$ $(4-5) \times 10^{14} \sim 1.5423 \times 10^{14} \mathrm{G}$, and $\frac{\dot{B}}{B^{3}}=0$ when $B=B_{\text {th }}$ because the directed EC reaction ceases. It's worth noting that the modified EC reaction always proceeds in the interior of a neutron star with any magnetic field strength, if the directed EC reaction exists, the modified EC reaction can be ignored (See Paper 1 and Paper 2). If the modified EC reaction dominates, the total magnetic decay rate $\frac{d B}{d t}$ (or $\dot{B}$ ) may be determined by many other factors, eg., Ohmic decay, ambipolar diffusion and Hall drift (Goldreich \& Reisenegger 1992; Rheinhardt \& Geppert 2003; ?). However, we mainly focus on the relation of $\frac{\dot{B}}{B^{3}}$ with $B$, rather than a specified way of magnetic field when the modified EC reaction dominates. Using the method of dimensional analysis, we produce the schematic diagrams of $\frac{\dot{B}}{B^{3}}$ as a function $B$ if the magnetic field decay timescale $t$ is given, shown as in Fig. 8 c. The curves in Fig. $8 d$ are produced by the superposition of the curves in Fig. $8 b$ and Fig. $8 c$ in a wide range of $B \sim 3.0 \times 10^{15}-10^{12} \mathrm{G}$. From Fig. $8 d$, the total change trend of $\frac{\dot{B}}{B^{3}}$ is that $\frac{\dot{B}}{B^{3}}$ increases with decreasing $B$. Observations show that for most pulsars, their dipole magnetic fields decay slowly during their lifetimes, and their observed braking indices $n$ are smaller than 3 . For young pulsars including AXPs, an obvious correlation has been proved between $n<3$ and the dipole magnetic fields $B$ as well as their decay rates $d B / d t$, which can be easily seen from the combination of Fig.8 with Eq.(23).

Among the known 12 AXPs (9 confirmed, 3 candidates), $1 \mathrm{E} 2259+586$ has the weakest dipole magnetic field, $B=5.9 \times 10^{14} \mathrm{G}$, the shortest period derivative, $\dot{P}=4.843 \times 10^{-13} \mathrm{~s} \mathrm{~s}^{-1}$, and the longest spindown or characteristic age, $t_{\text {Spin }}=230 \mathrm{kyr}$. All of these date suggests that the value of $n$ of $1 \mathrm{E} 2259+586$ is less than the ideal value of $n=3$. For $1 \mathrm{E} 2259+586$, its soft X-ray emission could be associated with accretion (White \& Marshall 1984; van Paradijs et al. 1995), which is beyond of our model, and the direct EC reaction ceases due to the weaker field $\left(B \ll B_{\text {th }}\right.$ ), however the modified EC reaction still occurs, from which weaker X-ray and weaker neutrino flux are produced. For this source, the weakest dipole magnetic field $B$ and super-low rates of decay of $B$ via the modified EC reaction may be the major causes that contribute to $n<3$.

In the above parts, we explain why all AXPs associated with SNRs appear older than they are. The reason why all SGRs associated with SNRs appear younger than their real ages is studied in the follows. Unlike AXPs, all SGRs can emit short bursts in the hard Xray/soft gamma-ray range with $E \sim 10^{39}-10^{41} \mathrm{erg}$ (Mereghetti 2008). Furthermore, giant flares and intermediate flares (or giant outbursts) were detected in SGRs associated with SNRs. Table 3 reports the details of giant/intermediate flares from these four SGRs associated with SNRs. In addition, other intermediate flares occurred in SGR 1900+14 on August 29, 


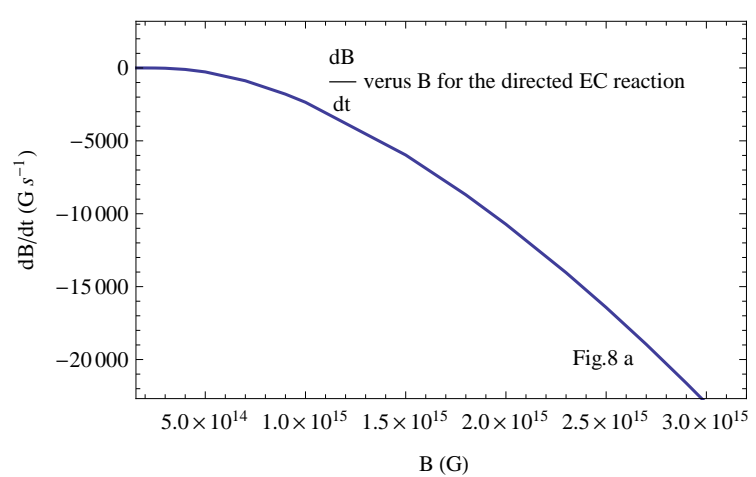

(a)

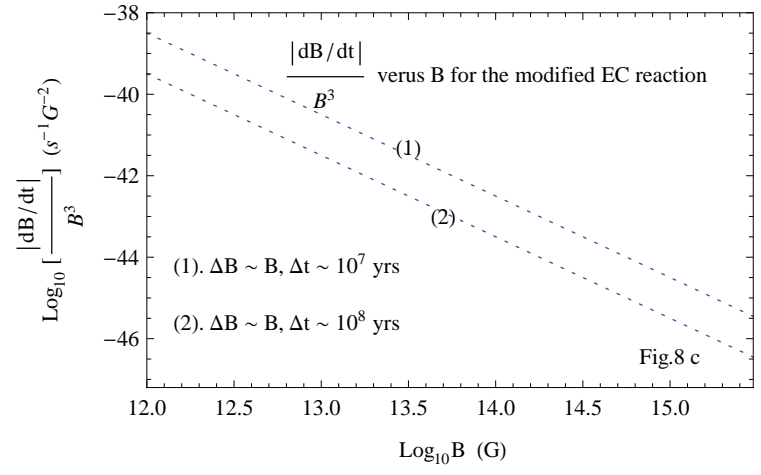

(c)

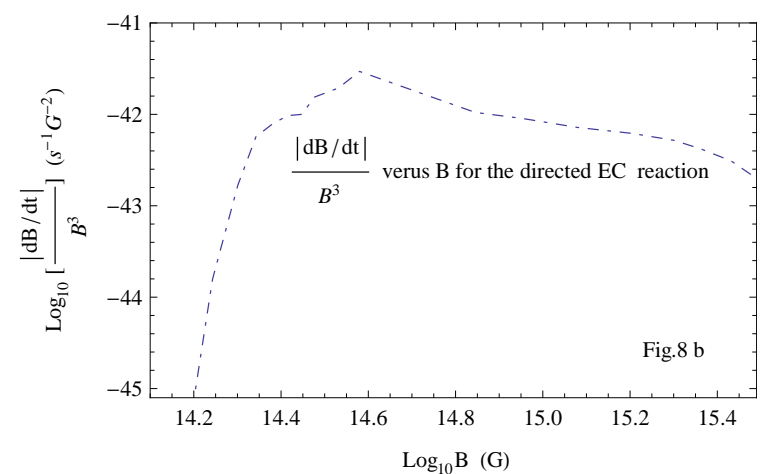

(b)

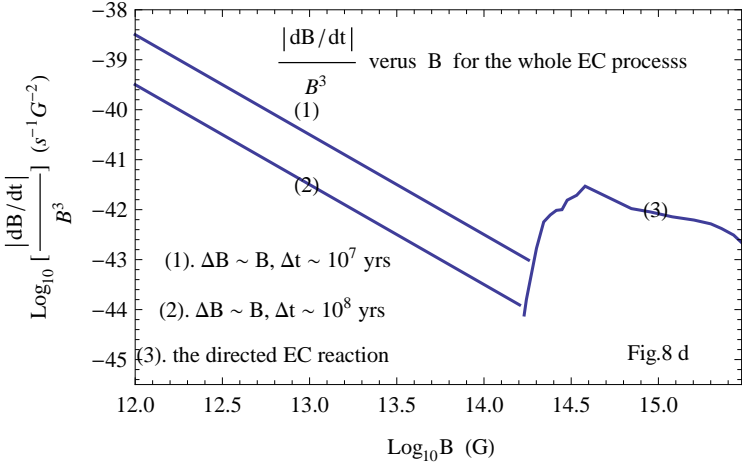

(d)

Fig. 8 The diagrams of $d B / d t-B$ and $\frac{\dot{B}}{B^{3}}-B$

1998 (Ibrahim et al. 2001), and on April 28, 2001 (Lenters et al. 2003). These two intermediate flares are no longer listed in Table 3 because of their relatively lower energies $\sim 10^{39}-10^{41} \mathrm{erg}$. The data in Table 3 implies a significant correlation between giant/intermediate flares and $t_{\mathrm{SNR}}>t_{\text {Spin }}$ for SGRs. The decay of diploe magnetic fields and giant/intermediate flares can conjointly affect spinning behaviors of SGRs associated with SNRs. However, we suppose that giant/ intermediate flares make SGRs look younger than they are. Now, an explanation of $t_{\mathrm{SNR}}>t_{\text {Spin }}$ for SGRs in the context of the star-quake model of magnetars (Thompson et al. 2002) is presented. The details are as follows:

Giant flares/bursts could be motivated by a largescale fracture of the crust, driven by magnetic stresses; the sudden crust's cracking sets the whole magnetar 'quaking', which results in a significant change in configuration of the dipole magnetic field (including magnetic field strength, magnetic field decay rate, the angle between magnetic axis and spin axis, moment of inertia, magnetic moment and so on). Such a change in configuration of the dipole magnetic field could give rise to unusual increases in spin-down torque as well as braking index $n$. Therefore, $\dot{P}$ also increases substantially, which can be illustrated by significant jumps in the pe- riod evolution of SGR 1900+14 after the 27 August 1998 giant flare (Marsden et al. 1999; Mereghetti et al. 2000). In short, for a SGR associated with a SNR, it is this change in configuration of the dipole magnetic field that could produce a significant deviation of $n(n>3)$ and cause the current value of $\dot{P}$ to be far larger than its mean value in history, the spin-down age will be far less than its real age. We can take SGR 1806-20 as an excellent example. From Table 3, the highest energy $E \sim(2-5) \times 10^{47}$ erg was released during the giant flare of December 27, 2004 from SGR 1806-20 which exceeded all previous giant/intermediate flares of SGRs. Among the known 23 magnetar, SGR 1806-20 has the strongest dipole magnetic field, $B=2.4 \times 10^{15} \mathrm{G}$, the largest period derivative, $\dot{P}=7.5 \times 10^{-10} \mathrm{~s} \mathrm{~s}^{-1}$, and the shortest spin-down or characteristic age, $t_{\text {Spin }}=0.16$ kyrs. All of this implies that the value of braking index of SGR 1806-20 is larger than the ideal value of $n=3$.

An alternative explanation that all SGRs associated with SNRs appear younger than their real ages is provided in the follows. Glitches (sudden frequency jumps of a magnitude $\Delta \Omega / \Omega \sim 10^{9}$ to $10^{6}$, accompanied by the jumps of spin-down rates with a magnitude of $\Delta \dot{\Omega} / \dot{\Omega} \sim 10^{-3}-10^{-2}$ )are common phenomenon in pulsars. After each glitch, a permanent increase in the pulsars spin-down rate usually happens, which causes 
Table 3 Giant/Intermediate flares from four SGRs.

\begin{tabular}{crrrr}
\hline Source & SGR $^{a} 0526-66$ & SGR $^{a} 1900+14$ & SGR $^{a}$ 1806-20 & SGR $^{b} 1627-41$ \\
\hline Date & March 5, 1979 & August 27, 1998 & December 27, 2004 & June 17, 1998 \\
Assumed Distance(kpc) & 55 & 15 & 15 & 5.8 \\
Peak Luminosity $(\mathrm{erg} / \mathrm{s})$ & $3.6 \times 10^{44}$ & $>1.5 \times 10^{44}$ & $(1.6 \sim 5) \times 10^{46}$ & $8 \times 10^{42}$ \\
Isotropic Energy $(\mathrm{erg})$ & $1.6 \times 10^{44}$ & $>8.3 \times 10^{44}$ & $(2 \sim 5) \times 10^{47}$ & $8 \times 10^{43}$ \\
\hline
\end{tabular}

Note: The sign 'a' denotes: The date are cited from (Mereghetti 2008). The sign 'b' denotes: The date are cited from (Mazets et al. 1999).

a slow increase in the pulsars surface polar magnetic field (be note, neither the period nor the spin-down rate is completely recovered although there is a relaxation after a glitch). Consequently, some radio pulsars with many active glitches may evolve into magnetars Lin \& Zhang 2004; Chen \& Li 2006). As we know, magnetars show many similarities with typical radio pulsars, including the properties of glitches. The amplitudes of glitches of SGRs are far larger than those of AXPs and radio pulsars (Mereghetti 2008). The glitches of SGRs associated with SNRs could be triggered by stars' 'quaking', contributing to magnetars' giant/intermediate flares (Mereghetti 2008). The significant changes of spin-down rates and dipole magnetic field strengthes before and after giant flares of SGRs (0526-66, 1806-20, 1900+14 and 1627-41) associated with SNRs are good indications of huge glitches happened in four SGRs though some of these huge glitches are 'missed'(not reported) (Pons \& Rea 2012). Therefore, a SGR's present spin-down rate may be much higher than its initial value, and its characteristic age may be shorter than its true age. With respect to AXPs with SNRs, on one hand, their dipole magnetic fields may also increase to a certain degree via glitches, on the other hand, the dipole magnetic fields rapidly decay via EC reaction, but the rates of increase are far less than the rates of decay. Thus, it's the dipolar magnetic field decay that plays an important role in making an AXP look older than it really is. It is worth noting that when a glitch happens, Eq.(23) no longer applies because the quantities of $\theta, I, R, \Omega($ or $P$ ) may vary to some extent, apart from an increases in $B$.

In ZX2011, authors suggested that the characteristic age of a NS is not available to estimate its real age, and the physically meaningful criterion to estimate $t_{\text {Real }}$ is the NS-SNR association. Their suggestions are in the same way applicable to magnetars associated with SNRs, according to our analysis above.

\section{Conclusions}

In this paper, based on our modified model, we carry out a study of the magnetic field decay of magnetars in SNRs. The main conclusions are as follows:

1. In the presence of superhigh magnetic fields, the values of $\Gamma$ calculated by $\rho_{\mathrm{e}}$ derived in circular cylindrical coordinates are less than those of $\Gamma^{\prime}$ calculated by $\rho_{\mathrm{e}}^{\prime}$ deduced in spherical coordinates, due to the quantization of Landau levels. Combining the relation of $\Gamma=K(B) \Gamma^{\prime}$ with Landau level-superfluid modified factor $\Lambda$ yields a modified second-order differential equation for a superhigh magnetic field $B$ and its evolutionary timescale $t$.

2. Calculations show that the maximum of the field's decay timescale, $t \approx 2.9507 \times 10^{6}$ yrs when $B_{0}=$ $3.0 \times 10^{15} \mathrm{G}$ and $T_{0}=2.60 \times 10^{8} \mathrm{~K}$ (without considering the modified Urca reactions). Assuming different initial internal temperatures, the superhigh magnetic fields may evolve on timescales $\left(10^{6} \sim 10^{7}\right)$ yrs for common magnetars.

3. On the basis of the results of the NS-SNR association of Zhang \& Xie (2011), we calculate the maximum initial internal magnetic fields of magnetar progenitors to be $\sim 2.0 \times 10^{14}-2.93 \times 10^{15} \mathrm{G}$, when $T_{0} \sim 2.60 \times 10^{8}$ K. If $T_{0} \sim 2.75 \times 10^{8}-1.75 \times 10^{8} \mathrm{~K}$, there still be $B_{\mathrm{i}} \sim\left(10^{14}-10^{15}\right) \mathrm{G}$, which are consistent with our model.

4. By means of statistical analysis, we found that an intense and significant positive correlation between $\log _{10}\left(\mathrm{SNR}\right.$ (Age)/Spindown(Age)) and $\log _{10} B$ for magnetars, and all AXPs associated with SNRs look older than their real ages, whereas all SGRs associated with SNRs appear younger than they are.

5. We tentatively investigate the equation of braking index $n$ under pure magnetodipole radiation, and produce schematic diagrams of $\frac{d B}{d t}-B$ and $\frac{\dot{B}}{B^{3}}-B$ in a wide range of $B \sim 3.0 \times 10^{15} \sim 10^{12} \mathrm{G}$. According to our magnetar model, braking index $n$ could be correlated with both the dipole magnetic field and its decay rate. 
6. 'The dipolar magnetic field decay plays a significant role in making a neutron star look older' suggested by Zhang and Xie (2011) is also applicable to magnetars. All AXPs may have experienced relatively 'normal' decay of their dipole magnetic fields, and thus have lower values of $n(n<3)$ and $\dot{P}$. In contrast, giant/intermediate flares were detected in SGRs associated with SNRs. Giant flares or huge glitches cause SGRs associated with SNRs spin down quickly, and make SGRs appear younger than their real ages.

Finally, due to the very little number of magnetars associated with SNRs, the above conclusions are tentative, and must be observationally validated.

Acknowledgements We thank the anonymous referee for the care in reading the manuscript and for useful comments which help us to improve this paper substantially, Prof. Xiang-Dong Li for his valuable suggestions on contents of this paper and helps on language, Profs. Yong-Feng Huang and Zi-Gao Dai for their helpful comments. This work is partly supported by Chinese National Science Foundation through grant No.10773005, National Basic Research Program of China (973 Program 2009CB824800), China Ministry of Science and Technology under State Key Development Program for Basic Research (2012CB821800), Knowledge Innovation Program of CAS $\mathrm{KJCX}_{2}-\mathrm{YW}-\mathrm{T09}$, Xinjiang Natural Science Foundation No.2009211B35, the Key Directional Project of CAS and NSFC under projects 10173020, 10673021, 10773005, 10778631 and 10903019. 


\section{References}

Aguilera, D. N., Pons, J. A., Miralles, J. A., 2008, Astrophys. J. Lett., 673, L167

Aharonian, F., Akhperjanian, A. G., Barres, de Almeida, U; et al., 2008, Astron. Astrophys.,486,829

Allen, M. P., Horvath, J. E., 1997, Astrophys. J., 488, 409

Allen, M. P., Horvath, J. E., 2004, Astrophys. J., 616, 346

Alpar, M. A., Ankay, A., Yazgan, E., 2001, Astrophys. J. Lett., 577, L61

Arras, P., Cumming, A., Thompson, C., 2004, Astrophys. J. Lett., 608, L49

Bardeen, J., Cooper, L. N., Schrieffer, J.R.,1957, Phys. Rev. 108,1175

Blandford, R. D., Romani, R. W., 1988, Mon. Not. R. Astron. Soc., 234, 57

Camilo, F., Ransom, S. M., Halpern, J. P., Reynolds, J., 2007, Astrophys. J. Lett., 666, L93

Chen, W. C., Li, X. D., 2006, Astron. Astrophys., 450, L1

Corbel, S., Chapuis, C., Damc, T. M., Durouchoux, P.,1999, Astrophys. J. Lett., 526, L29

Duncan R. C., Thompson C., 1992, Astrophys. J., 392, L9

Duncan R. C., Thompson C., In: Rothschild R.E., Lingenfelter R.E. (eds.) High-Velocity Neutron Stars and Gamma-Ray Bursts. AIP Conference Proc., Vol. 366, p. 111. AIP Press, New York (1996)

Duncan, R. C., in Fifth Hunstville Gamma-Ray Burst Symposium, AIP Conference Proceedings No. 526, ed. R. M. Kippen, R. S. Mallozzi, \& G. J. Fishman, American Institute of Physics, New York (2000), arXiv:astro-ph/0002442

Elgarøy, Ø., et al., 1996, Phys. Rev. Lett., 77, 1482

Fahlman, G. G., Gregory, P. C., 1981, Nature, 293, 202

Gaensler, B. M., Gotthelf, E. V., Vasisht, G., 1999,Astrophys. J. Lett., 526, L37

Gaensler, B. M., Gotthelf, E. V., Vasisht, G., 2001,Astrophys. J., 559, 963

Gelfand J. D., Gaensler, B. M., 2007, Astrophys. J., 667, 1111 arXiv:0706.1054

Green, D. A., 1989, Mon. Not. R. Astron. Soc., 238, 737

Gao, Z. F., Wang, N., Yuan, J. P., et al., 2011a, Astrophys. Space Sci.,332, 129

Gao, Z. F., Wang, N., Yuan, J. P., et al., 2011b, Astrophys. Space Sci., 333, 427

Gao, Z. F., Wang, N., Song, D. L., et al., 2011c, Astrophys. Space Sci., 334, 281

Gao, Z. F., Peng, Q. H., Wang, N., et al., 2011d, Astrophys. Space Sci., 336, 427

Goldreich, P., Reisenegger, A., 1992, Astrophys. J., 395, 250

Gunn, J. E., Ostriker, J. P., 1970, Astrophys. J., 160, 979

Halpern, J. P., Gotthelf, E. V., 2010, Astrophys. J., 725, 1384

Han, J. L., 1997, Astron. Astrophys., 489, 485

Heyl, J. S., Hernquist, L., 1997, Astrophys. J. Lett., 489, L67

Heyl, J. S., Kulkarni, S. R., 1998, Astrophys. J. Lett., 506, L61

Hurley, K., Kouveliotou, C., Woods, P., et al., 1999, Astrophys. J. Lett., 510, L107

Horiuchi, S., Suwa, Y., Takami, H., et al., 2008, Mon. Not. R. Astron. Soc., 391, 1893
Horvath, J. E., Allen, M. P., 2011, Res. Astron. Astrophys. 11,625

Ibrahim, A. I., Strohmayer, T. E., Woods, P. M., et al., 2001, Astrophys. J., 558, 237

Ibrahim, A. I., Markwardt, C. B., Swank, J. H, et al., 2004, Astrophys. J. Lett., 609, L1

Klose, S., Henden, A. A., Geppert, U., et al. 2004, Astrophys. J. Lett., 609, L13, arXiv:astro-ph/0405299

Kouveliotou, C., Fishman, G. J., Meegan, C. A., et al., 1994, Nature, 368, 125

Kulkarni, S. R., Frail, D. A.,1993,Nature, 365, 33

Kulkarni, S. R., Kaplan, D. L., Marshall, H. L., et al., 2003, Astrophys. J., 585, 948

Lenters, G. T., Woods, P. M., Goupell, J. E., et al., 2003, Astrophys. J., 587, 761

Lin, J. R., Zhang, S. N., 2004, Astrophys. J. Lett., 615, L133

Manchester, R. N., Taylor, J. H.,: Pulsars. San Francisco, CA(USA): W. H. Freeman, 281p

Marsden, D., Rothschild, R. E., Lingenfelter, R. E., 1999, Astrophys. J. Lett., 520, 1107

Marsden, D., Lingenfelter, R. E., Rothschild, R. E., et al., 2001, Astrophys. J., 550, 397

Mazets, E. P., Aptekar, R. L., Butterworth, P. S., 1999, Astrophys. J. Lett., 519, L151

Mereghetti, S., Cremonesi, D., Feroci, M., Tavani, M., 2000, Astron. Astrophys., 361, 240

Menou, K., Perna, R., Hernquist, L., 2001, Astrophys. J. Lett., 554, L63

Mereghetti, S., Götz, D., Mirabel, I. F., et al., 2005, Astron. Astrophys., 433, L9

Mereghetti, S., 2008, Astron. Astrophys. Rev., 15, 225

Narayan, R., Ostriker, J. P., 1990, Astrophys. J., 352, 222

Ostriker, J. P., Gunn, J. E., 1969, Astrophys. J., 157, 1395

Peng, Q. H., Huang, K. L., Huang, J. H., 1982, Astron. Astrophys., 107, 258

Peng, Q. H., Tong H., 2007, Mon. Not. R. Astron. Soc., 378, 159

Peng Qiu He., Tong Hao., arXiv: 0911.2066v1 [astro-ph.HE] 11 Nov 2009, $10^{\text {th }}$ Symposium on Nuclei in the Cosmos, 27 July-1 August 2008 Mackinac Island, Michigan,USA

Pons, J. A., Link, B., Miralles, J. A., \& Geppert, U., 2007, Phys. Rev. Lett, 98, 071101

Pons, J. A., Miralles, J. A., Geppert, U., 2009, Astron. Astrophys., 496, 207

Pons, J. A., Rea, N., 2012,Astrophys. J. Lett., 750, 6

Rheinhardt, M., Geppert, U., 2003, Phys. Rev. Lett., 88, L101

Ridley, J. P., Lorimer, D. R., 2010, Mon. Not. R. Astron. Soc., 404, 1081

Rho, J., Petre, R., 1997, Astrophys. J.,484, 828

Sanbonmatsu, K. Y., Herfand, D. J., 1992,Astrophys. J., 104, 2189

Shapiro, S. L., Teukolsky, S. A., 1983, 'Black holes, white drarfs, and neutron stars' John Wiley \& Sons, New York

Shull, J. M., Fesen, R. A., Saken, J. M., 1989, Astrophys. J., 346,860

Ternov, I., Lysov, B., Korovina, L., 1965, Moscow Univ. Phys. Bull. 5, 58

Thompson, C., Duncan, R. C., 1993, Astrophys. J., 543, 340

Thompson, C., Duncan, R. C., 1996, Astrophys. J., 473, 322 
Thompson, C., Duncan, R. C., Woods, P. M., 2000, Astrophys. J., 543, 340

Thompson, C., Lyutikov, M., Kulkami, S. R., 2002, Astrophys. J., 574, 332

van Paradijs, J., Taam, R. E., van den Heuvel, E. P. J., 1995, Astron. Astrophys., 299, 41

Vasisht, G., Kulkarni, S. R., Frail, D. A., et al., 2000, Astrophys. J. Lett., 431, L35

Vasisht, G., Gotthelf, E. V., 1997, Astrophys. J. Lett., 486, L129

Vasisht, G., Gotthelf, E. V., Torri, K., et al., 2000, Astrophys. J. Lett., 542, L49

Wacheter, S., Patel, S., Kouveliotou, C., et al., 2004, Astrophys. J., 615,887

White, N. E., Marshall, F. E., 1984,Astrophys. J., 281, 354

Wu, F., Xu, R. X., Gil, J., 2003, Astron. Astrophys., 409, 641

Xu, R. X., Qiao, G. J., 2001, Astrophys. J. Lett., 561, L85

Xu, R. X., Tao, D. J., Yang, Y., 2006, Mon. Not. R. Astron. Soc., 373, L85

Yakovlev, D. G., Kaminker A. D., Gnedin O. Y., et al., 2001, Phys. Rep., 354, 1

Zhang, B., Xu, R. X., Qiao, G. J., 2000, Astrophys. J. Lett., 545, L127

Zhang, S. N., Xie, Y., 9th Pacific Rim Conference on Stellar Astrophysics, Lijiang, China in 14-20 April 2011., ASP Conference Series, Vol. 451. Edited by S. Qain, K. Leung, L. Zhu, and S. Kwok. San Francisco: Astronomical Society of the Pacific, p.231 (2011). arXiv:1110.3154v1[astroph.HE] 


\section{Appendix}

\section{A An important assumption on the ${ }^{3} P_{2}$ neutron Cooper pairs}

The formation of Cooper pairs is a universal quantum-mechanical phenomenon of condensation in superfluids or superconductors. As pointed out in the original BCS work (Bardeen et al. 1957), pairing occurs basically between fermion states in the vicinity of the Fermi surface. Owing to pairing correlations, there is a major change in the low-energy spectrum of the system: A finite energy gap between its ground state and first excited state appears, and then the system will be subjected to a phase transition to a superfluid (or superconductor) state below a critical temperature.

From the analysis in Papers 1-4, the ${ }^{3} P_{2}$ neutron Cooper pairs can be destroyed by the outgoing EC neutrons easily. However, up to the present, the physics community has not yet produced the calculation of the collision probability at which an outgoing EC neutron destroys one ${ }^{3} P_{2}$ Cooper pair, due to special circumstances inside neutron stars, e.g., high temperatures, high-density matter, ultra-strong magnetic fields, and so on.

As a matter of fact, in the process of EC, for each degenerate species (electrons, protons, neutrons and neutrinos), only a fraction $\left(\sim k T / E_{\mathrm{F}}(i)\right)$ of particles lying in the vicinity of the Fermi surface can effectively contribute to the EC rate, $\Gamma$. Now, let us carry out the following evaluation: The number of neutrons participating in EC per unit volume, $n_{\mathrm{n}}^{\prime}$, is computed as

$n_{\mathrm{n}}^{\prime}=\frac{k T}{E_{\mathrm{F}}^{\prime}(n)} \int \rho_{\mathrm{n}} d E_{\mathrm{n}}=\frac{k T}{E_{\mathrm{F}}^{\prime}(n)} \times \frac{8 \pi \sqrt{2} m_{\mathrm{n}}^{3 / 2}}{h^{3}} \int_{E_{\mathrm{F}}^{\prime}(n)}^{\left\langle E_{\mathrm{n}}\right\rangle} E_{\mathrm{n}}^{\frac{1}{2}} d E_{\mathrm{n}}$.

As an illustration, we can arbitrarily assume $B=3.0 \times 10^{15} \mathrm{G}$ and $T=2.78 \times 10^{8} \mathrm{~K}$. From Paper 4, we obtain the following relations: $\left\langle E_{\mathrm{n}}\right\rangle=73.57 \mathrm{MeV}$, and $E_{\mathrm{F}}^{\prime}(n)=60 \mathrm{MeV}$ when $B=3.0 \times 10^{15} \mathrm{G}$. Eq.(A1) gives the value $n_{\mathrm{n}}^{\prime} \sim 2.377 \times 10^{34} \mathrm{~cm}^{-3}\left(\rho=\rho_{0}\right)$. Since the values we assumed are the possible maximum values of $B$ and $T$, the average value of $n_{\mathrm{n}}^{\prime}$ will be less than this value evaluated $\left(2.377 \times 10^{34} \mathrm{~cm}^{-3}\right)$, obviously. In the interior of a neutron star where the anisotropic ${ }^{3} P_{2}$ neutron superfluid exists, the neutron number density $n_{\mathrm{n}}=1.78 \times 10^{38} \mathrm{~cm}^{-3}$ when $\rho=\rho_{0}$ (Shapiro \& Teukolsky 1983), which indicates that both the number of neutrons and the number of the ${ }^{3} P_{2}$ neutron Cooper pairs per unit volume are far larger than the number of these newly formed (EC) neutrons per unit volume. Based on the above analysis, we may make a feasible assumption that each outgoing high-energy EC neutron can destroy one ${ }^{3} P_{2}$ neutron Cooper pair.

\section{B Necessary corrections and improvements in our previous work}

For the purpose of improving our magnetar model, we have checked our previous research in an all-round way. In this part, we make several necessary corrections in Paper 3, and provide key improvements in Paper 4.

In Paper 3, we derived the formulae for $E_{\mathrm{F}}(\mathrm{e})$ in superhigh magnetic fields, and concluded that the stronger the magnetic fields, the higher the electron Fermi energy becomes. However, the coefficient $\frac{2}{3}$ in Eq.(15) was wrongly treated as $\frac{3}{2}$ in the subsequent calculations, causing the formulae of $E_{\mathrm{F}}(\mathrm{e})$ deviate the actual case slightly. We honestly apologize to readers for our mistake. Now, the necessary corrections in Paper 3 are presented as follows: $\frac{3 \pi}{B^{*}}$ in Eqs.(16-19) must be replaced by $\frac{4 \pi}{3 B^{*}} ; \frac{(3 \pi)^{2}}{16 B^{*}}$ in Eq.(20) in Paper 3 must be replaced by $\frac{\pi^{2}}{4 B^{*}}$; Eq.(23) in Paper 3 can be rewritten as

$E_{\mathrm{F}}(\mathrm{e})=43.44\left[\frac{Y_{\mathrm{e}}}{0.0535} \frac{\rho}{\rho_{0}} \frac{B}{B_{\mathrm{cr}}}\right]^{\frac{1}{4}} \mathrm{MeV} \quad\left(B^{*} \geq 1\right)$

To our pleasure, the higher value of $Y_{\mathrm{e}}=0.12$ (see Paper 4) will be replaced by the lower value $Y_{\mathrm{e}}=0.0535$. The later is slightly larger than the mean value of $Y_{\mathrm{e}}$ of a neutron star, $Y_{\mathrm{e}}=0.05$, and thus is plausible. Furthermore, the corrections of Eq.(20) and Eq.(24) in Paper 3 don't affect the calculated results of Paper 4. Now, some values of $E_{\mathrm{F}}(\mathrm{e})$ of Table 2 in Paper 3 are modified, shown as in Table 4. From the analysis in Paper 3, when $E_{F}(\mathrm{e}) \geq 5$ $\mathrm{MeV}$, the second term on the right of Eq.(20) can be ignored. This suggest that the second term on the right of 
Table 4 The relation of $E_{\mathrm{F}}(\mathrm{e})$ and $B$.

\begin{tabular}{lrrrrr}
\hline Name & $Y_{\mathrm{e}}$ & $E_{r m F}(1)$ & $E_{r m F}(2)$ & $E_{\mathrm{F}}(3)$ & $E_{\mathrm{F}}(4)$ \\
& & $B^{*}=0$ & $B^{*}=1$ & $B^{*}=10$ & $B^{*}=100$ \\
${ }_{26}^{56} \mathrm{Fe}$ & 0.4643 & 0.95 & $\mathbf{1 . 3 5}$ & $\mathbf{3 . 2 0}$ & $\mathbf{8 . 7 7}$ \\
\hline${ }_{28}^{62} \mathrm{Ni}$ & 0.4516 & 2.61 & $\mathbf{2 . 4 8}$ & $\mathbf{4 . 5 8}$ & $\mathbf{1 2 . 0 1}$ \\
${ }_{28}^{64} \mathrm{Ni}$ & 0.4375 & 4.31 & & $\mathbf{6 . 4 4}$ & $\mathbf{1 3 . 4 9}$ \\
${ }_{28}^{66} \mathrm{Ni}$ & 0.4242 & 4.45 & & $\mathbf{6 . 5 4}$ & $\mathrm{NO}$ \\
${ }_{386}^{86} \mathrm{Kr}$ & 0.4186 & 5.66 & & & $\mathbf{1 4 . 4 9}$ \\
${ }_{34}^{84} \mathrm{Se}$ & 0.4048 & 8.49 & & & $\mathbf{1 8 . 8 4}$ \\
${ }_{32}^{82} \mathrm{Ge}$ & 0.3902 & 11.44 & & & $\mathbf{2 1 . 7 5}$ \\
${ }_{30}^{80} \mathrm{Zn}$ & 0.3750 & 14.08 & & & $\mathbf{2 7 . 6 2}$ \\
\hline
\end{tabular}

Note: This table is cited from Table 2 of Paper 3. All the corrections are denoted in boldface.

Eq.(16) also can be ignored. Thus, the electron energy state density can be approximately expressed as

$$
\begin{aligned}
N_{\text {pha }} \approx \quad & \frac{4 \pi}{3 B^{*}}\left(\frac{m_{\mathrm{e}} c}{h}\right)^{3} \int_{0}^{\frac{E_{\mathrm{F}}(\mathrm{e})}{m_{\mathrm{e}} c^{2}}}\left[\left(\frac{E_{\mathrm{F}}(\mathrm{e})}{m_{\mathrm{e}} c^{2}}\right)^{2}\right. \\
& \left.-1-\left(\frac{p_{z}}{m_{\mathrm{e}} c}\right)^{2}\right]^{\frac{3}{2}} d\left(\frac{p_{z}}{m_{\mathrm{e}} c}\right) \cdot(26)
\end{aligned}
$$

Differentiating Eq.(26) gives the following expression:

$\frac{d N_{\mathrm{pha}}}{d E_{\mathrm{e}}} d E_{\mathrm{e}} \simeq \frac{4 \pi}{3 B^{*}}\left(\frac{m_{\mathrm{e}} c}{h}\right)^{3} \frac{1}{m_{\mathrm{e}} c^{2}}\left[\left(\frac{E_{\mathrm{F}}(\mathrm{e})}{m_{\mathrm{e}} c^{2}}\right)^{2}-1-\left(\frac{E_{\mathrm{e}}}{m_{\mathrm{e}} c^{2}}\right)^{2}\right]^{\frac{3}{2}} d E_{\mathrm{e}} \cdot(27)$

Simplifying Eq.(27) and using the relation $\rho_{\mathrm{e}}=\frac{d n_{\mathrm{e}}}{d E_{\mathrm{e}}}=\frac{d N_{\mathrm{pha}}}{d E_{\mathrm{e}}}$, we gain

$$
\begin{aligned}
& \rho_{\mathrm{e}}=\frac{4}{3} \frac{\pi}{B^{*}}\left(\frac{m_{\mathrm{e}} c}{h}\right)^{3} \frac{1}{m_{\mathrm{e}} c^{2}}\left[\left(\frac{E_{\mathrm{F}}(\mathrm{e})}{m_{\mathrm{e}} c^{2}}\right)^{2}-1-\left(\frac{E_{\mathrm{e}}}{m_{\mathrm{e}} c^{2}}\right)^{2}\right]^{\frac{3}{2}} \\
& =\frac{1}{3 B^{*}\left(2 \pi^{2} \hbar^{3} c^{3}\right)} \frac{1}{m_{\mathrm{e}} c^{2}}\left[E_{\mathrm{F}}^{2}(\mathrm{e})-0.261-E_{\mathrm{e}}^{2}\right]^{\frac{3}{2}},(28)
\end{aligned}
$$

where $m_{\mathrm{e}} c^{2}=0.511 \mathrm{MeV}$ is used. Taking into account of gravitation redshift and utilizing Eq.(25) and Eq.(28), Eq.(16) and Eq.(19) in Paper 4 are modified as

$L_{\mathrm{X}}^{\infty}=\zeta(B, T)\left(1-r_{\mathrm{g}} / R\right) \frac{d E}{d t}$,

and

$$
\begin{aligned}
L_{\mathrm{X}}^{\infty} \simeq \quad & \Lambda(B, T) \zeta(B, T)\left(1-r_{\mathrm{g}} / R\right) \\
& \times \frac{4}{3} \pi R_{5}^{3} \frac{2 \pi}{\hbar} \frac{G_{\mathrm{F}}^{2} C_{\mathrm{V}}^{2}\left(1+3 a^{2}\right)}{2 \pi^{2} \hbar^{3} c^{3}} \frac{8 \pi \sqrt{2} m_{\mathrm{n}}^{\frac{3}{2}}}{h^{3}} \\
& \times \frac{\left(1.60 \times 10^{-6}\right)^{9.5}}{\left(2 \pi^{2} \hbar^{3} c^{3}\right) 3 B^{*} m_{\mathrm{e}} c^{2}} \int_{E_{\mathrm{F}}^{\prime}(\mathrm{n})}^{\left\langle E_{\mathrm{n}}\right\rangle} E_{\mathrm{n}}^{\frac{1}{2}}\left\langle E_{\mathrm{n}}\right\rangle d E_{\mathrm{n}} \\
& \times \int_{Q}^{E_{\mathrm{F}}(\mathrm{e})}\left[E_{\mathrm{F}}^{2}(\mathrm{e})-0.261-E_{\mathrm{e}}^{2}\right]^{\frac{3}{2}}\left(E_{\mathrm{e}}-Q\right)^{3} d E_{\mathrm{e}},(30)
\end{aligned}
$$

respectively, where $L_{\mathrm{X}}^{\infty}$ is the apparent soft X-ray luminosity measured by a distant observer or the redshifted soft X-ray luminosity; $r_{\mathrm{g}}=2 \mathrm{GM} / \mathrm{c}^{2}=2.95 \mathrm{M} / M_{\odot} \mathrm{km}$ is the Schwarzschild radius (we assume $R=10^{6} \mathrm{~cm}$ and $M=1.4 M_{\odot}$ for a canonical magnetar). We introduce the parameter $\phi(B, T)$ to denote $\Lambda(B, T) \zeta(B, T)$ in Eq.(30), the value of $\phi(B, T)$ of a magnetar can be evaluated by combining Eq.(B6) with Table 4 of Paper 4. Fig.9 shows 


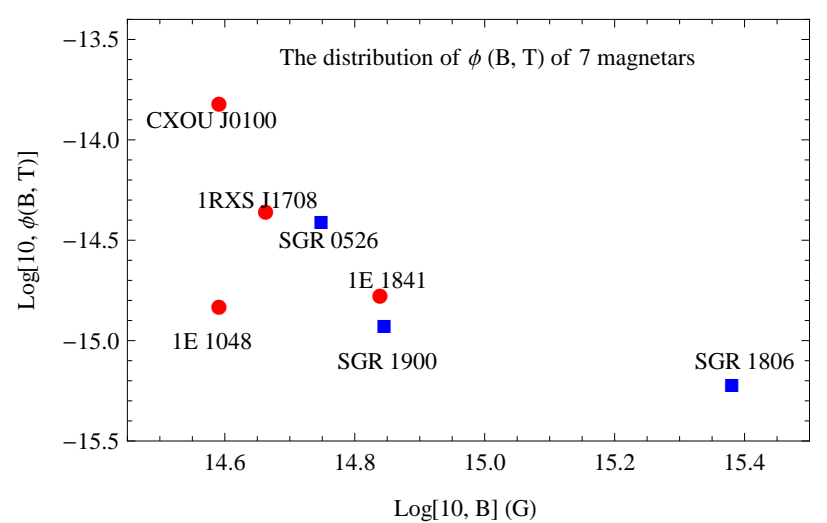

Fig. 9 The distribution of $\phi(B, T)$ of 7 canonic magnetars when $B_{0}=3.0 \times 10^{15} \mathrm{G}$ and $T_{0}=2.6 \times 10^{8} \mathrm{~K}$. The range of $B$ is assumed to be $\left(1.80 \times 10^{14} \sim 3.0 \times 10^{15} \mathrm{G}\right.$, arbitrarily. Circles and squares are for AXPs and SGRS, respectively.

the distribution of $\phi(B, T)$ of 7 canonic magnetars, whose persistent soft X-ray luminosities should not be less than their rotational energy loss rates $d E / d t$ (Paper 4). It is worth noting that the fitting curve of $\phi(B, T)$ as a function of $B$ and $T$ cannot be obtained from Fig.9 because of the very little number of canonic magnetars. For each canonical magnetar with a given soft X-ray luminosity, its value of $\phi$ is mainly determined by $B$, and is insensitive to $T$, though $\phi$ is a function of $B$ and $T$. The mean value of $\phi(B, T)$ of 7 magnetars is calculated to be $2.54 \times 10^{-15}$ by using the expression of $\langle\phi\rangle=\frac{\sum B_{i} \phi_{i}}{\sum B_{i}}$. Combining Eq.(7) with $\langle\phi\rangle$ gives

$$
\begin{aligned}
\phi(B, T) \approx \quad & \frac{2.54 \times 10^{-15}}{3.198 \times 10^{-14}}\left(2.60388 \times 10^{-14}\right. \\
& \left.+1.98233 \times 10^{-30} B\right)\left(\frac{T_{0}}{2.6 \times 10^{8} \mathrm{~K}}\right)^{4} \\
& \times \exp \left[\frac{-0.048 \mathrm{MeV}}{k}\left(\frac{1}{T_{0}}-\frac{1}{2.6 \times 10^{8} \mathrm{~K}}\right)\right] \\
& =\left(2.068 \times 10^{-15}+1.5747 \times 10^{-31} B\right) \\
& \times\left(\frac{T_{0}}{2.6 \times 10^{8} \mathrm{~K}}\right)^{4} \\
& \times \exp \left[\frac{-0.048 \mathrm{MeV}}{k}\left(\frac{1}{T_{0}}-\frac{1}{2.6 \times 10^{8} \mathrm{~K}}\right)\right] .(31)
\end{aligned}
$$

Inserting Eq.(31) into Eq.(30), we calculate the values of $L_{\mathrm{X}}^{\infty}$ in superhigh magnetic fields, $L_{\mathrm{X}}^{\infty} \sim\left(5.457 \times 10^{28}-\right.$ $\left.3.834 \times 10^{37}\right) \mathrm{erg} \mathrm{s}^{-1}$, corresponding to $B \sim\left(1.8 \times 10^{14}-3.0 \times 10^{15}\right)$ G. Furthermore, the calculated results of $L_{\mathrm{X}}^{\infty}$ are compared with those of $L_{\mathrm{X}}$ of Paper 4, shown as in Fig.10 From Fig.10, the values of $L_{\mathrm{X}}^{\infty}$ are slightly less than those of $L_{\mathrm{X}}$, the main reason for this is that the factor of gravitation redshift is considered in calculating $L_{\mathrm{X}}^{\infty}$. 


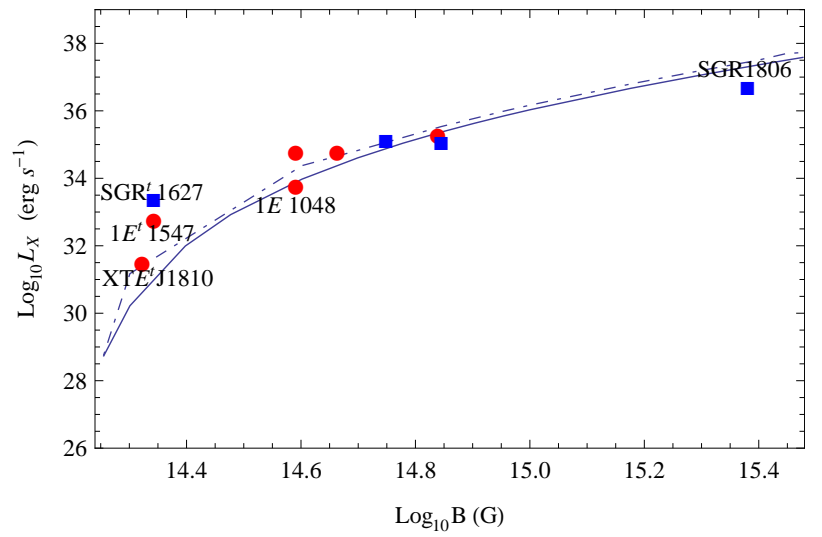

Fig. 10 The diagrams of soft X-ray luminosity as a function of magnetic field strength $B$ when $B_{0}=3.0 \times 10^{15} \mathrm{G}$ and $T_{0}=2.6 \times 10^{8} \mathrm{~K}$. The range of $B$ is assumed to be $\left(1.80 \times 10^{14} \sim 3.0 \times 10^{15} \mathrm{G}\right.$, arbitrarily. Circles and squares are for AXPs and SGRS, respectively. Solidline and dot-dashedline are for the modified luminosities and unmodified luminosities (Paper 4), respectively. 